\title{
The clinical implementation of optical spectroscopy \\ in colorectal cancer surgery
}

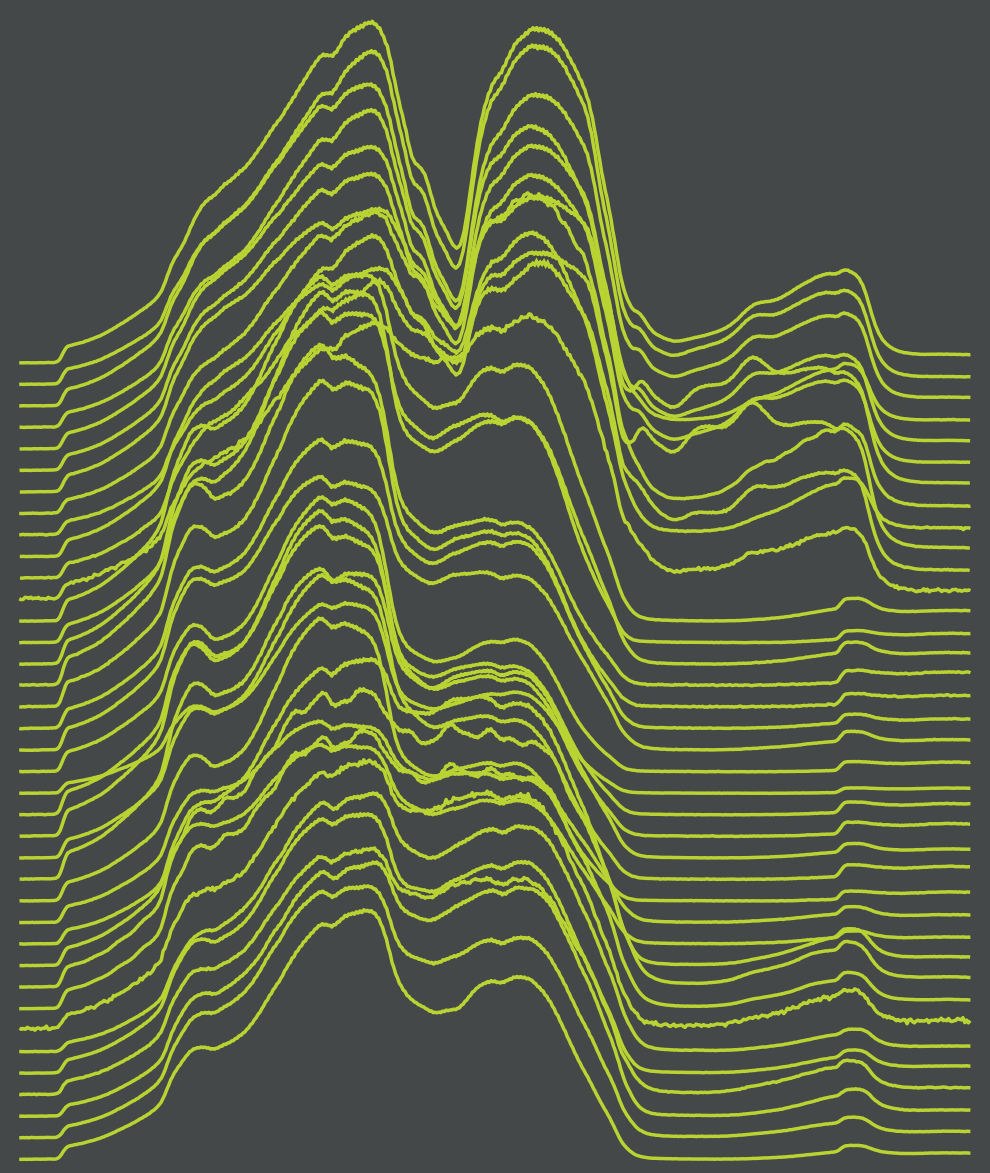

Elisabeth J.M. Baltussen 


\section{THE CLINICAL IMPLEMENTATION OF OPTICAL SPECTROSCOPY IN COLORECTAL CANCER SURGERY}


This disertation was approved by:

Supervisor:

prof. dr. T.J.M. Ruers

Co-supervisors:

prof. dr. H.J.C.M. Sterenborg

dr. K.F.D. Kuhlmann

Cover image: @ E.J.M. Baltussen

Printing: Ipskamp Printing

ISBN: 978-90-365-4961-5en

DOI: $10.3990 / 1.9789036549615$

URL: https://doi.org/10.3990/1.9789036549615

(C) 2020 E.J.M. Baltussen, The Netherlands. All rights reserved. No parts of this thesis may be reproduced, stored in a retrieval system or transmitted in any form or by any means without permission of the author. Alle rechten voorbehouden. Niets uit deze uitgave mag worden vermenigvuldigd, in enige vorm of op enige wijze, zonder voorafgaande schriftelijke toestemming van de auteur 


\title{
THE CLINICAL IMPLEMENTATION OF OPTICAL SPECTROSCOPY IN COLORECTAL CANCER SURGERY
}

\author{
DISSERTATION
}

\author{
To obtain \\ the degree of doctor at the University of Twente, \\ on the authority of the rector magnificus, \\ prof. dr. T.T.M. Palstra, \\ on account of the decision of the Doctorate Board, \\ to be publicly defended \\ on Wednesday $4^{\text {th }}$ of March 2020 at 14.45
}

by

Elisabeth Johanna Maria Baltussen

born on $4^{\text {th }}$ of June 1989

in Rosmalen, The Netherlands 


\section{GRADUATION COMMITTEE:}

Chairman/secretary: prof. dr. J.L. Herek

Supervisor:

Co-supervisors:

Members: prof. dr. T.J.M. Ruers, The Netherlands Cancer Institute

prof. dr. H.J.C.M. Sterenborg

dr. K.F.D. Kuhlmann

prof. dr. B.H.W. Hendriks

prof. dr. G.L. Beets

prof. dr. C.G. Slump

dr. H.L. Offerhaus

dr. R.M. Verdaasdonk 


\section{Table of contents}

1 Introduction 7

2 Diffuse reflectance spectroscopy as a tool for real-time tissue assessment during colorectal cancer surgery

3 Hyperspectral imaging for tissue classification, a way towards smart laparoscopic colorectal surgery

4 Using diffuse reflectance spectroscopy to distinguish tumor tissue from fibrosis in rectal cancer patients as a guide to surgery

5 Tissue diagnosis during colorectal cancer surgery using optical sensing: an in vivo study

6 Comparing in vivo and ex vivo fiberoptic diffuse reflectance spectroscopy in colorectal cancer

7 Optimizing tissue classification algorithms in colorectal cancer using diffuse reflectance

8 General Discussion

Englishe summary

Nederlandse samenvatting (Dutch summary)

Acknowledgments

PhD Portfolio

Curriculum Vitae 
Chapter 1

Introduction 


\subsection{Colorectal cancer}

\section{Colorectal cancer}

Colorectal cancer is the third most common cancer worldwide, covering more than $10 \%$ of the new cancer cases in 2018. After lung cancer, colorectal cancer is the second cause of cancer related deaths, with more than 880.000 deaths estimated in 2018 [1, 2]. About one third of the colorectal cancers are located in the rectum [3].

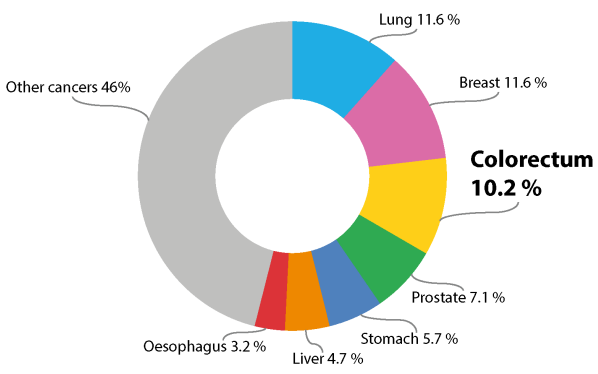

(a) Incidence

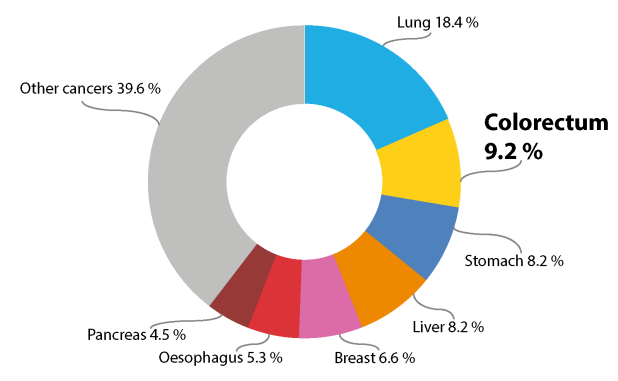

(b) Mortality

Figure 1.1: Incidence and mortality of all cancer types for men and women combined

Surgery is the standard treatment for colon and rectum cancer. In advanced stage colon cancer, surgery is often combined with chemotherapy. Neoadjuvant chemotherapy is given to downstage the tumor and adjuvant chemotherapy to reduce the risk of recurrence. For advanced stage rectal cancer, surgery is mostly combined with neoadjuvant (chemo)radiotherapy [4]. There are three common options for neoadjuvant treatment in rectal cancer. The first option is short course radiotherapy of 25 Gy in 5 fractions followed by immediate or delayed surgery. The second option is 45-50 Gy radiotherapy given during 5 weeks in combination with a radiosensitizer and surgery about three months after the start of the radiotherapy. The final option is 25 Gy in 5 fractions followed by 6 cycles of chemotherapy and srugery 6-8 weeks after the final chemotherapy dose. This option is only used for patients with metastases or an increased chance on metastases.

In surgery, especially for rectal cancer surgery, a balance should be found by the surgeon between complete removal of the tumor and sparing healthy surrounding tissue. Extending surgical resection margins to reduce the risk of a tumour positive margins can lead to damage of vital and functional surrounding structures, such as the bladder and hypogastric plexus. Currently, after rectal cancer 
surgery bladder dysfunction occurs in $20-30 \%$ of the patients and sexual disorders in approximately $30 \%$. It is obvious that these complications have significant impact on the patients' quality of life. In rectal cancer surgery, complete removal of the tumor is pathologically assessed by determining the circumferential resection margin (CRM), i.e. the shortest distance between tumor and resected surface, measured over the entire circumferential resection plane. A positive CRM is defined as a CRM of less than $1 \mathrm{~mm}$. It was found to be one of the most important prognostic factors for local recurrence and overall survival [5-7]. A positive margin after rectal cancer surgery is found in about $20 \%$ of the cases, with increasing risks for advanced tumors (2\% stage I, $14 \%$ stage II and $33 \%$ stage III) and for tumors lower in the rectum (16\% versus $26 \%$ ) [7]. With the introduction of the total mesorectal excision (TME) the number of positive resection margins decreased to about $10 \%$. Neoadjuvant chemo- and radiotherapy decreased the amount of positive resection margins over just radiotherapy with $9 \%$ from 13 to $4 \%$ [5].

The main challenge during rectal cancer surgery is to determine the optimal resection plane. This resection plane should provide a sufficient CRM and spare as much healthy surrounding tissue as possible. To determine the optimal resection plane, real-time tissue classification is essential. During rectal cancer surgery visual classification of tissue by the surgeon is often impaired due to the limited space in the pelvic cavity. Due to the increase in laparoscopic surgeries over the past years, also tactile feedback used by the surgeon for tissue classification is impaired, making it more difficult for the surgeon to define the optimal resection plane. Therefore, intraoperative real-time technology that can be used for tissue classification during colorectal cancer surgery would be of great benefit to the surgeon and could improve the outcome of the surgery.

\subsection{Diffuse reflectance spectroscopy}

Diffuse reflectance spectroscopy (DRS) is a technology that can be used for intraoperative real-time tissue classification. DRS is based on the interaction of light with tissue. In DRS an optical fingerprint of the examined tissue is obtained by sending light into the tissue and collecting light that is reemitted the tissue surface. To obtain this fingerprint, light over a broad wavelength range is send through a fiber to the tissue. In the tissue, the light will undergo several interactions with the tissue, which include scattering and absorption. Scattering will cause the direction of individual light particles to change and absorption will pre- 
vent part of the light to be reemitted. Part of the light will be scattered back to the surface, where it can be detected with a second fiber. In DRS the distance between the emitting and receiving fibers determines the depth at which the tissue is mainly assessed. With an increase in fiber distance, the depth of the path of the detected light will increase as well. The exact measurement depth depends mainly on the fiber distance, but to a certain extent also on the tissue constituents and the wavelength of the light, and, as a rule of thumb, is roughly the same as the fiber distance [8].

The spectral composition of the light that is collected at the surface of the tissue will be different from the light that was send into the tissue. These changes depend on the tissue constituents and structure and thereby give the optical fingerprint of the tissue. Light of a wavelength that is mainly absorbed by the tissue, will have low intensity in the received signal whereas wavelengths that are mainly scattered, will generally have a higher intensity in the received signal. The spectra collected at the surface of the tissue can be used to classify the measured tissue types [9]. Classification of the DRS spectra can be performed in several ways. First, the entire unprocessed spectrum can be used for classification. In addition, to reduce the number of features used for the classification, several feature extraction or selection methods can be used like principal component analysis or discriminant analysis. Another option is to translate the spectra into optical properties of the tissue, extracting the absorption and scattering coefficients for each wavelength. This method requires prior knowledge on the interaction between light and tissue [10]. Finally, these optical properties can be translated into biological parameters related to tissue constituents. This method requires even more prior knowledge, because the absorption spectra of all tissue constituents present in the tissue should be known in advance [9, 11, 12].

DRS has been used before in several studies to distinguish tumor tissue from healthy surrounding tissues, for instance in breast, liver, lung and head and neck cancer [13-18]. In colorectal cancer DRS has also been used successfully before. The main focus of DRS in colorectal cancer has been on the use in colonoscopy. These studies all focused on the visual wavelength region, in which hemoglobin is the main absorber. All studies found that it is possible to distinguish premalignant and tumor tissue in the lumen from healthy lumen in the colon using just the hemoglobin concentration and blood saturation [19-23]. Two studies reported sensitivity and specificity values on the differentiation between healthy and tumor tissue, sensitivities of 80 and $92 \%$ and specificities of 75 and $78 \%$ were ob- 
tained [21, 22]. The main difference between a colonoscopy setting and surgery is the side from which the bowel is assessed. In a colonoscopy setting, measurements are performed from inside the lumen, where only healthy mucosal tissue and premalignant or tumor tissue are present. In a surgical setting, however, the bowel is assessed from outside the lumen. In the pelvic area many different types of healthy tissue are present, making tissue classification more complex. Furthermore, in a surgical setting the presence of blood on the surface cannot be easily controlled, making tissue classification based just on blood parameters less reliable. Some studies have focused on the use of DRS in colorectal cancer surgery [24-26]. All these studies were performed ex vivo and spectra were obtained in the visual and near-infrared wavelength range. The sensitivity and specificity obtained in these studies were $62 \%$ and $90 \%$ [26] respectively, and $95 \%$ and $88 \%$ [25], respectively.

\section{Hyperspectral imaging}

Hyperspectral reflection imaging $(\mathrm{HSI})$ is based on the same interaction of light as DRS. In HSI the tissue is illuminated directly with a broadband light source. This light interacts with the tissue, through scattering, absorption and reflection. After several interactions part of the light will be reflected from the surface of the tissue were the light can be detected by a hyperspectral camera [27]. The hyperspectral camera obtains $2 \mathrm{D}$ images of the tissue over several wavelengths, which results in a 3D data cube. In this data cube two dimensions contain the spatial information and the third dimension contains the spectral information. In $\mathrm{HSI}$, the spectra contain information from several depths, depending on the tissue constituents and wavelength [27]. Light from more shallow depths will have more influence on the spectra because the signal from these shallow depths will have more intensity left due to less interactions with the tissue compared to signal from larger depths.

There are several ways to obtain the 3D data cube. First of all, by spatial scanning in which one spatial dimension and the entire spectral dimension is obtained at a time. The second spatial dimension is obtained by moving the tissue underneath the camera [27]. The second option is spectral scanning in which the two spatial dimensions are imaged at a time and the spectral dimension is obtained by changing filters in front of the camera chip. For this option the tissue should be fixed with respect to the camera during acquisition, because movement of the tissue will cause defects in the obtained spectra [27]. The third option is a spa- 
tiospectral scanning system, in which the wavelengths are divided over one spatial dimension. One image includes both spatial dimensions, but for one of the spatial dimensions the wavelength at which it is obtained changes. To obtain a complete 3D data cube, the tissue can be moved with respect to the camera, or an element in the camera can be moved to change spatial locations the wavelengths [28]. Finally, a snapshot camera can be used, in which all spatial dimensions and a selected set of wavelengths are obtained at once [29]. These snapshot cameras seem the best solution but these systems have high manufacturing costs.

Tissue classification using HSI is comparable to DRS, because the signals obtained are similar. Therefore, also in HSI entire unprocessed spectra can be used for tissue classification, as well as feature extraction and selection methods to reduces the number of features used [27]. The extraction of optical properties of the tissue from HSI spectra is possible as well. However, for the extraction of biological parameters, a different algorithm should be used, because of the differences in measurement set-up between DRS and HSI measurements. Different than in DRS, in HSI classification spatial information can be used as well. Because an entire image is obtained, information from neighboring pixels can be useful in the classification.

In previous studies $\mathrm{HSI}$ was used to distinguish tumor tissue from healthy tissue in cervix [30], breast [31], skin [32], tongue [33], head and neck [34, 35] and gastric cancer [36]. The application of HSI in colorectal cancer has mainly been focused on the classification of hematoxylin-eosin (H\&E) pathology slides [41, 37-40] and tissue classification during colonoscopy [42-44]. In H\&E slide classification, HSI is used to support the pathologist in the diagnosis of colorectal cancer [41, 3739]. In colonoscopy the application, $\mathrm{HSI}$ is used to distinguish tumor tissue from healthy tissue during colonoscopy. All studies were performed in the visual wavelength range and mainly focused on differences in the amount of blood present in healthy and tumor tissue. The sensitivity and specificity values obtained were varied slightly between $72.5 \%$ and $96.9 \%$, and $78 \%$ and $91.5 \%$ respectively [ $42-$ 44].

Even though HSI obtains similar information of the tissue to DRS, there are several major differences. First of all, in DRS only point measurements can be obtained, whereas with $\mathrm{HSI}$ an entire image is taken at once. Furthermore, for DRS measurements contact between the probe and the tissue is required to perform measurements, whereas for HSI a certain distance is required to obtain an image of the entire tissue sample. Finally, in DRS the sampling depth is mainly 
determined by the distance between the emitting and receiving fiber. In HSI the acquired signal contains information of all different depths, with the emphasis on the more shallow depths, because this signal is least decreased by tissue interactions. In addition, the average sampling depth changes strongly with optical properties and thus with wavelength.

\subsection{Outline of this thesis}

The research described in this thesis is focused on the use of DRS and HSI in colorectal cancer surgery to develop a technique that can be used real-time during surgery to distinguish healthy tissue from tumor tissue. Due to the different nature of DRS and $\mathrm{HSI}$, their final application will differ slightly. For DRS the ultimate goal is to incorporate the technique into a surgical device. When DRS is combined with the cutting device used by the surgeon, direct feedback on the tissue in front of the cutting device will be available for the surgeon. The final application of HSI will be in the laparoscopic setting, where the laparoscopic camera can be replaced by a hyperspectral camera, thereby providing tissue classification of the entire image real-time. In order to come to these devices, first the discriminating ability of DRS and $\mathrm{HSI}$ in colorectal cancer need to be examined.

In Chapter 2 and Chapter 3, this is done in the most controlled setting. In Chapter 2 DRS measurements are performed on ex vivo tissue samples of healthy tissue and tumor tissue provided by the pathologist. Similar samples were also imaged by the hyperspectral cameras, to obtain a first insight in the abilities of $\mathrm{HSI}$ in colorectal cancer surgery. The results of this research are presented in

\section{Chapter 3.}

The next step is taken in Chapter 4 where DRS measurements are obtained on entire ex vivo specimens. By performing the measurements on entire tissue specimen instead of tissue samples a step is taken towards the ultimate use during surgery. Furthermore, fibrotic tissue is included to the analysis to obtain a more complete classification of all non-cancerous tissue surrounding colorectal tumors.

In Chapter 5, an in vivo study using DRS during colorectal cancer surgery is performed. In this study measurements were obtained in 32 patients. These measurements were analyzed postoperatively and correlated to the pathology results to obtain a tissue classification based on DRS spectra.

The final chapters of this thesis are more focused on the analyses of the spectra 
obtained. In Chapter 6, a comparison in made between measurements obtained in vivo and measurements obtained ex vivo. Because data acquisition ex vivo is more easy than data acquisition in vivo, in case both datasets are comparable, the more easy ex vivo studies can be used to obtain large datasets in the future. In Chapter 7, different techniques for analysis will be compared to optimize data analysis of optical measurements in colorectal cancer.

In Chapter $\mathbf{8}$ a general discussion on all results in this thesis will be given together with a future perspective. 


\section{References}

[1] J. Ferlay, M. Ervik, and F. Lam, "Global Cancer Observatory: Cancer Today." https://gco.iarc.fr/today/data/factsheets/cancers/10_8_9-Colorectum-fact-sheet.pdf, 2018.

[2] F. Bray et al., "Global cancer statistics 2018: GLOBOCAN estimates of incidence and mortality worldwide for 36 cancers in 185 countries," CA: A Cancer Journal for Clinicians, vol. 68, pp. 394424, nov 2018.

[3] R. Glynne-Jones et al., "Rectal cancer: ESMO Clinical Practice Guidelines for diagnosis, treatment and follow-upt," Annals of Oncology, vol. 28, pp. iv22-iv40, jul 2017.

[4] K. D. Miller et al., "Cancer treatment and survivorship statistics, 2016," CA: A Cancer Journal for Clinicians, vol. 66, pp. 271-289, jun 2016.

[5] I. D. Nagtegaal and P. Quirke, "What Is the Role for the Circumferential Margin in the Modern Treatment of Rectal Cancer?," Journal of Clinical Oncology, vol. 26, no. 2, pp. 303-312, 2008.

[6] C. Simillis et al., "A systematic review to assess resection margin status after abdominoperineal excision and pelvic exenteration for rectal cancer," Annals of surgery, vol. 265, no. 2, pp. 291299, 2017.

[7] I. D. Nagtegaal et al., "Circumferential margin involvement is still an important predictor of local recurrence in rectal carcinoma: not one millimeter but two millimeters is the limit," The American journal of surgical pathology, vol. 26, no. 3, pp. 350-357, 2002.

[8] A. J. Gomes and V. Backman, "Algorithm for automated selection of application-specific fiberoptic reflectance probes," Journal of Biomedical Optics, vol. 18, p. 27012, feb 2013.

[9] T. M. Bydlon et al., "Chromophore based analyses of steady-state diffuse reflectance spectroscopy: current status and perspectives for clinical adoption," Journal of Biophotonics, vol. 8, pp. 9-24, jan 2015.

[10] T. J. Farrell, M. S. Patterson, and B. Wilson, "A diffusion theory model of spatially resolved, steady-state diffuse reflectance for the noninvasive determination of tissue optical properties in vivo," Medical Physics, vol. 19, no. 4, pp. 879-888, 1992.

[11] R. Nachabé et al., "Estimation of biological chromophores using diffuse optical spectroscopy: benefit of extending the UV-VIS wavelength range to include 1000 to $1600 \mathrm{~nm}$," Biomedical Optics Express, vol. 1, no. 5, pp. 1432-1442, 2010.

[12] R. Nachabé et al., "Estimation of lipid and water concentrations in scattering media with diffuse optical spectroscopy from 900 to $1,600 \mathrm{~nm}$.," Journal of biomedical optics, vol. 15, no. 3 , p. 037015, 2015.

[13] D. J. Evers et al., "Optical sensing for tumor detection in the liver," European Journal of Surgical Oncology, vol. 39, no. 1, pp. 68-75, 2013. 
[14] E. Tanis et al., "In vivo tumor identification of colorectal liver metastases with diffuse reflectance and fluorescence spectroscopy," Lasers in Surgery and Medicine, vol. 48, pp. 820-827, nov 2016.

[15] J. W. Spliethoff et al., "Improved identification of peripheral lung tumors by using diffuse reflectance and fluorescence spectroscopy," Lung Cancer, vol. 80, no. 2, pp. 165-171, 2013.

[16] L. L. Boer et al., "Fat/water ratios measured with diffuse reflectance spectroscopy to detect breast tumor boundaries," Breast Cancer Research and Treatment, vol. 152, no. 3, pp. 509-518, 2015.

[17] J. S. Soares et al., "Diagnostic power of diffuse reflectance spectroscopy for targeted detection of breast lesions with microcalcifications," Proceedings of the National Academy of Sciences, vol. 110, pp. 471 LP - 476, jan 2013.

[18] S. G. Brouwer de Koning et al., "Toward complete oral cavity cancer resection using a handheld diffuse reflectance spectroscopy probe," Journal of Biomedical Optics, vol. 23, pp. 1-9, oct 2018.

[19] G. Zonios et al., "Diffuse reflectance spectroscopy of human adenomatous colon polyps in vivo," Applied optics, vol. 38, no. 31, pp. 6628-6637, 1999.

[20] H.-W. Wang et al., "Diffuse reflectance spectroscopy detects increased hemoglobin concentration and decreased oxygenation during colon carcinogenesis from normal to malignant tumors.," Optics express, vol. 17, no. 4, pp. 2805-17, 2009.

[21] H. K. Roy et al., "Spectroscopic Microvascular Blood Detection From the Endoscopically Normal Colonic Mucosa: Biomarker for Neoplasia Risk," Gastroenterology, vol. 135, no. 4, pp. 10691078, 2008.

[22] A. Dhar et al., "Elastic scattering spectroscopy for the diagnosis of colonic lesions: initial results of a novel optical biopsy technique," Gastrointestinal Endoscopy, vol. 63, pp. 257-261, feb 2006.

[23] J. R Mourant et al., Elastic scattering spectroscopy as a diagnostic tool for differentiating pathologies in the gastrointestinal tract: Preliminary testing, vol. 1. apr 1996.

[24] R. M. Schols, P. Dunias, F. P. Wieringa, and L. P. S. Stassen, "Multispectral characterization of tissues encountered during laparoscopic colorectal surgery," Medical Engineering and Physics, vol. 35, no. 7, pp. 1044-1050, 2013.

[25] G. C. Langhout et al., "Differentiation of healthy and malignant tissue in colon cancer patients using optical spectroscopy: A tool for image-guided surgery," Lasers in Surgery and Medicine, vol. 47, no. 7, pp. 559-565, 2015.

[26] R. J. Beaulieu et al., "Automated diagnosis of colon cancer using hyperspectral sensing," The International Journal of Medical Robotics and Computer Assisted Surgery, vol. 14, p. e1897, jun 2018.

[27] G. Lu and B. Fei, "Medical hyperspectral imaging: a review," Journal of Biomedical Optics, vol. 19, p. 10901, jan 2014.

[28] S. Grusche, "Basic slit spectroscope reveals three-dimensional scenes through diagonal slices of hyperspectral cubes," Appl. Opt., vol. 53, pp. 4594-4603, jul 2014. 
[29] W. R. Johnson et al., "Snapshot hyperspectral imaging in ophthalmology," Journal of Biomedical Optics, vol. 12, pp. 1-7, jan 2007.

[30] J. M. Benavides et al., "Multispectral digital colposcopy for in vivo detection of cervical cancer," Optics Express, vol. 11, no. 10, pp. 1223-1236, 2003.

[31] E. Kho et al., "Hyperspectral imaging for resection margin assessment during cancer surgery," Clinical Cancer Research, pp. clincanres-2089, 2019.

[32] D. Hattery, M. Hassan, S. Demos, and A. Gandjbakhche, "Hyperspectral imaging of Kaposi's Sarcoma for disease assessment and treatment monitoring," in Applied Imagery Pattern Recognition Workshop, 2002. Proceedings., pp. 124-130, 2002.

[33] Z. Liu, H. Wang, and Q. Li, "Tongue Tumor Detection in Medical Hyperspectral Images," Sensors (Basel, Switzerland), vol. 12, pp. 162-174, dec 2012.

[34] G. Lu et al., "Detection of Head and Neck Cancer in Surgical Specimens Using Quantitative Hyperspectral Imaging," Clinical Cancer Research, vol. 23, pp. 5426 LP - 5436, sep 2017.

[35] B. Fei et al., "Label-free reflectance hyperspectral imaging for tumor margin assessment: a pilot study on surgical specimens of cancer patients," Journal of Biomedical Optics, vol. 22, p. 1, aug 2017.

[36] H. Akbari et al., "Cancer detection using infrared hyperspectral imaging," Cancer Science, vol. 102, pp. 852-857, apr 2011.

[37] M. Maggioni et al., "Hyperspectral microscopic analysis of normal, benign and carcinoma microarray tissue sections," vol. 6091, p. 60910I, International Society for Optics and Photonics, feb 2006 .

[38] K. Masood and N. M. N. M. Rajpoot, "Classification of colon biopsy samples by spatial analysis of a single spectral band from its hyperspectral cube," 2007.

[39] K. Masood and N. M. N. M. Rajpoot, "Spatial analysis for colon biopsy classification from hyperspectral imagery," 2008.

[40] S. Rathore, M. Hussain, A. Ali, and A. Khan, "A Recent Survey on Colon Cancer Detection Techniques," IEEE/ACM Transactions on Computational Biology and Bioinformatics, vol. 10, no. 3, pp. 545-563, 2013.

[41] K. Rajpoot and N. Rajpoot, "SVM Optimization for Hyperspectral Colon Tissue Cell Classification," in Medical Image Computing and Computer-Assisted Intervention - MICCAI 2004 (C. Barillot, D. R. Haynor, and P. Hellier, eds.), (Berlin, Heidelberg), pp. 829-837, Springer Berlin Heidelberg, 2004.

[42] E. Claridge and D. Hidović-Rowe, "Model Based Inversion for Deriving Maps of Histological Parameters Characteristic of Cancer From Ex-Vivo Multispectral Images of the Colon," IEEE Transactions on Medical Imaging, vol. 33, no. 4, pp. 822-835, 2014.

[43] Z. Han et al., "In vivo use of hyperspectral imaging to develop a noncontact endoscopic diagnosis support system for malignant colorectal tumors," vol. 21, pp. 16001-16008, 2016. 
[44] R. Kumashiro et al., "Integrated Endoscopic System Based on Optical Imaging and Hyperspectral Data Analysis for Colorectal Cancer Detection," Anticancer Research, vol. 36, pp. 3925-3932, aug 2016. 


\section{Chapter 2}

\section{Diffuse reflectance spectroscopy as a tool for real-time tissue assessment during colorectal cancer surgery}

Elisabeth J.M. Baltussen, Petur Snaebjornsson, Susan G. Brouwer de Koning, Henricus J.C.M. Sterenborg, Arend G.J. Aalbers, Niels F.M. Kok, Geerard L. Beets, Benno H.W. Hendriks, Koert F.D. Kuhlmann, Theo J.M. Ruers, Journal of Biomedical Optics, 2017, Volume 22 (10) 


\section{Abstract}

Colorectal surgery is the standard treatment for patients with colorectal cancer. To overcome two of the main challenges, the circumferential resection margin and postoperative complications, real-time tissue assessment could be of great benefit during surgery. In this ex vivo study, diffuse reflectance spectroscopy (DRS) was used to differentiate tumor tissue from healthy surrounding tissues in patients with colorectal neoplasia. DRS spectra were obtained from tumor tissue, healthy colon or rectal wall and fat tissue, for every patient. Data were randomly divided into train (80\%) and test (20\%) sets. After spectral band selection, the spectra were classified using a quadratic classifier and a linear support vector machine. Of the 38 included patients, 36 had colorectal cancer, 2 had an adenoma. When the classifiers were applied to the test set, colorectal cancer could be discriminated from healthy tissue with an overall accuracy of $0.95( \pm 0.03)$. This study demonstrates the possibility to separate colorectal cancer from healthy surrounding tissue by applying diffuse reflectance spectroscopy. High classification accuracies were obtained both in homogeneous and inhomogeneous tissues. This is a fundamental step towards the development of a tool for real-time in-vivo tissue assessment during colorectal surgery. 


\subsection{Background}

Colorectal cancer is the third most common cancer worldwide and ranks as the fourth cause of death due to cancer [1]. About $30 \%$ of the cases are located in the rectum [2]. For patients with rectal cancer, surgery, frequently combined with radiotherapy or chemo-radiotherapy, is the standard of care [3]. The delicate balance between complete removal of the tumor and the sparing of vital surrounding structures is of utmost importance in rectal cancer surgery. Perioperative tissue differentiation is complex, especially when there is fibrosis after preoperative chemo radiation. Inadequate tumor recognition can result in tumor positive resection margins, generally defined as tumor tissue within $1 \mathrm{~mm}$ of the circumferential resection margin (CRM) [4]. In patients with a positive CRM, the risk of local tumor recurrence is significantly increased and survival is compromised [5]. Although complete tumor removal is essential, damage to vital surrounding structures should be prevented during surgery. Damage to structures such as nerves may cause bladder and sexual dysfunction [6,7]. Currently, there is no intraoperative technique available for the assessment of the CRM or identification of surrounding structures, such as nerve bundles, except for the possibility to submit tissue for frozen section assessment. Real-time tissue differentiation during surgery may aid the surgeon to choose the most optimal resection plane, enabling radical tumor resection while sparing vital healthy tissue.

Optical tissue sensing by diffuse reflectance spectroscopy (DRS) may offer the possibility for real time tissue differentiation during surgery. In DRS, broadband white light is sent through a fiber into the tissue. After interaction with the tissue, the light is collected and analyzed for spectral changes. These changes are highly specific for the absorption and scattering characteristics of the individual tissue types. In this way the different tissue types can be distinguished based on differences in the reflected spectrum [8]. DRS has already been applied in several other studies to differentiate tumor tissue from healthy surrounding tissues, e.g. in lung and breast [9-11].

Although DRS has extensively been studied in many tissue types, data on the application of DRS in colorectal cancer are limited. DRS has mostly been evaluated during colonoscopy for the detection and characterization of pre-malignant and malignant tissue changes [12-16]. However, in colonoscopy the differentiation that needs to be made is between healthy epithelium and tumor tissue as assessed from inside the lumen. In contrast, during surgery the requirements for 
tissue characterization are different and tumor tissue has to be identified from the outside of the bowel wall. Hence, differentiation has to be made between tumor tissue, fatty tissue and healthy bowel wall. Only a few studies have applied DRS in a surgical setting for rectal cancer $[17,18]$. In a study from our group by Langhout et al., tumor tissue could be distinguished from healthy tissue, colon wall, epiploic fat and mesocolic tissue, with an accuracy of $91 \%$ [17]. An analytical model was used to obtain concentrations of the constituents of the tissue. Based on the concentrations the measurements are classified. This analytical model assumes that a semi-infinite homogenous optical medium is measured. However, the colorectal wall is a layered structure and thus not homogenous limiting the applicability of the analytical model to this problem as it leads to unstable and inaccurate fit results. In addition, in our earlier study by Langhout et al. it was not possible, based on histology, to determine inhomogeneity at the measurement locations, e.g. at tumor border locations with more than one tissue type.

Before taking the next step to in vivo measurements, we wanted to eliminate these limitations by quantifying the inhomogeneity of the sample volume as well as using a classification technique less sensitive to such inhomogeneity. To this end we developed a method for more precise correlation between measurement location and histopathology as well as a tissue classification method less sensitive to inhomogeneity of the measurement site.

\subsection{Materials and methods}

\subsubsection{Study protocol}

In this ex vivo study, patients from the Antoni van Leeuwenhoek - Netherlands Cancer Institute (Amsterdam, the Netherlands) and the Slotervaart Medical Centre (Amsterdam, the Netherlands) were included. The study was performed under approval of the protocol by the hospitals ethics review board. All patients were undergoing surgery for colorectal cancer.

Immediately after surgical resection, three tissue samples were taken from the specimen - tumor tissue, healthy colon or rectal wall and (peri-colorectal) fat and were placed into separate pathology cassettes. Within 1 hour after resection, DRS measurements were performed on all three tissue samples. Measurements of healthy colon wall were always performed on the muscular layer, i.e. the muscularis propria. For the microscopic verification of the DRS measurement locations 


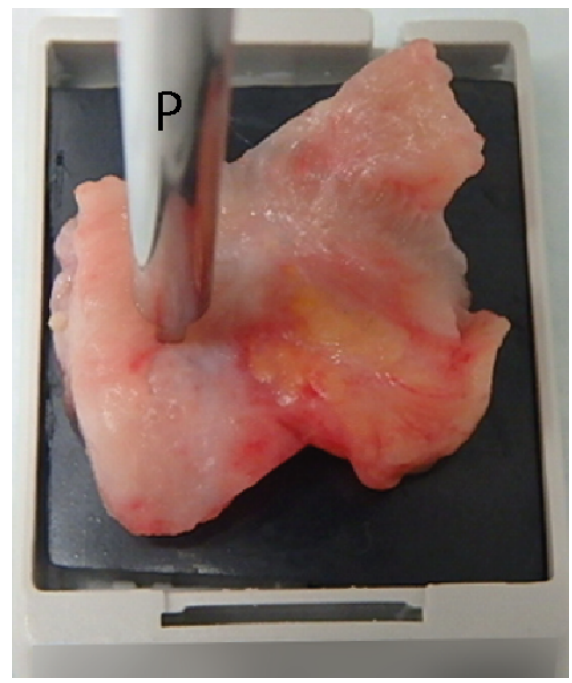

(a) RGB image

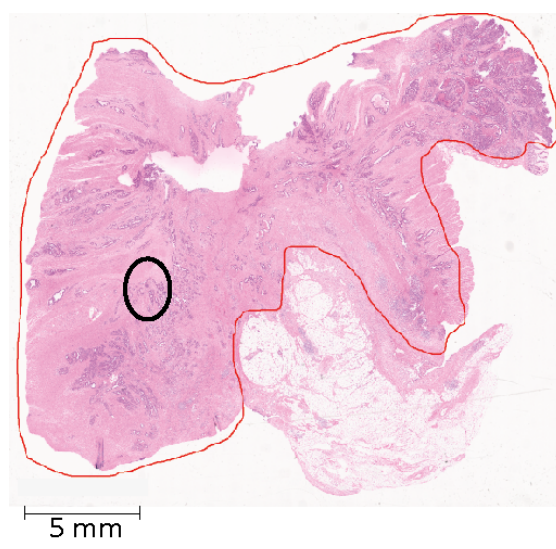

(b) Pathology slide

Figure 2.1: Match of RGB image with the measurement probe $(P)$ and pathology slide. In the pathology slide the measurement location is indicated by the black circle and the tumor area is indicated by the red line.

afterwards, the tissue samples were kept in the pathology cassettes. Furthermore, an overview image and images of each measurement recording the exact measurement locations (Fig. 1) were captured by an RGB-camera. Per patient, three measurements per location were performed, in at least three different locations per tissue type. The three measurements per location were averaged before the analysis. After acquisition of all spectra, the tissue samples were returned to the pathology department where the samples were further processed according to standard protocols. The resulting slides were examined by a pathologist, who marked the various tissue types. These annotated pathology slides were visually correlated with the RGB images taken from the measurement locations to identify the histological tissue type at each measured location (Fig. 2.1).

Measurements on locations, which were on microscopic analysis proven to be fat, healthy colorectal wall and tumor, were included in the further analysis. Measurements were excluded when the measurement location was not present in the pathology slide. Locations at the border of two tissue types were initially excluded from the analysis to reduce bias. At a later stage these locations were analyzed separately. 


\subsubsection{Diffuse Reflectance Spectroscopy system and probe}

The DRS system consists of a Tungsten halogen broadband light source (360$2500 \mathrm{~nm}$ ) with an embedded shutter and two spectrometers. Together, the two spectrometers cover the visible and near-infrared light range; the first spectrometer resolves light in the visible range 400-1100 nm (Andor Technology, DU420ABRDD), while the second resolves light in the near-infrared range $900-1700 \mathrm{~nm}$ (Andor Technology, DU492A-1.7) (Fig. 2.2). The spectrometers are controlled by custom LabView software (National Instruments) to acquire the data. The DRS system used in this study has been extensively described previously together with the calibration used for the system [19, 20].

In all patients, the same probe, shown in Fig. 2.2, was used to obtain the DRS measurements. The probe consists of three optical fibers with core diameter of $200 \mu \mathrm{m}$. One of the three fibers was used to transport the light from the source to the tissue, while the other two were used to transport the light from the tissue to one of both spectrometers. The distance between the two collecting fibers and the delivering fiber is $2 \mathrm{~mm}$. This gives a mean sampling depth of about $2 \mathrm{~mm}$ (max 4mm).

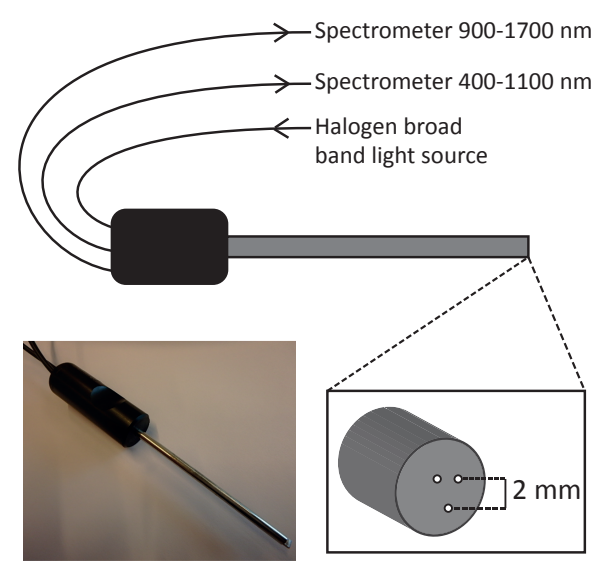

Figure 2.2: Probe used in this study. At the top a schematic image of the probe is shown. The bottom left shows an image of the probe. The bottom right shows a schematic image of the tip. The distance between the two collecting and the delivering fiber is $2 \mathrm{~mm}$. 


\subsubsection{Data analysis}

All analyses were performed using the perClass toolbox (Academic version 5.0, PR Sys design, Delft, the Netherlands) in Matlab (version 8.5, MathWorks Inc., Natick, Massachusetts, United States). To create and test a classifier, the data was first divided randomly into a train and test set. The data was split such that measurements of one patient were not divided between train and test set: $80 \%$ of the patients were used for training the classifier and the remaining $20 \%$ were used for testing the classifier.

Before training the classifier, the number of features used for the classification was reduced with the use of spectral bands. The spectral bands are selected using k-means clustering and consist of continuous wavelengths with similar spectral intensity. The mean intensity per spectral band is used as input for the further classification method. After determination of the spectral bands, two different classifiers were trained to separate the three different tissue types. First, a quadratic classifier was used to separate the measurements on fat tissue from the measurements on healthy colorectal wall and tumor tissue. Thereafter, a linear support vector machine (SVM) was used to distinguish measurements on healthy colorectal wall from tumor tissue. The SVM was optimized using an ROC curve created with a tenfold cross-validation using the train data. The optimal point selected in the ROC curve is the point that maximizes the mean accuracy. The final classifier pipeline included the spectral band selection, the quadratic classifier and the linear SVM (Fig. 2.3).

After training the classifiers, the classifier pipeline was applied to the test dataset to assess the performance of the classifier pipeline on separating the data of the different tissue types. The training and testing of the classifier was repeated ten times, with different randomly selected train and test sets, to obtain a more precise accuracy.

Finally, the dataset, consisting of all measurement locations containing one tissue type, was used to train the classifier pipeline once more. In clinical practice, there is a chance of measuring more than one tissue type in a single measurement. To evaluate the accuracy of the developed classifiers in such circumstances where only part of the measurement volume contained tumor tissue, the measurements that contained more than one tissue type, were further analyzed using this final developed classifier pipeline. The proportions of the different types of tissue involved were estimated based on visual correlation with the pathology slides. 


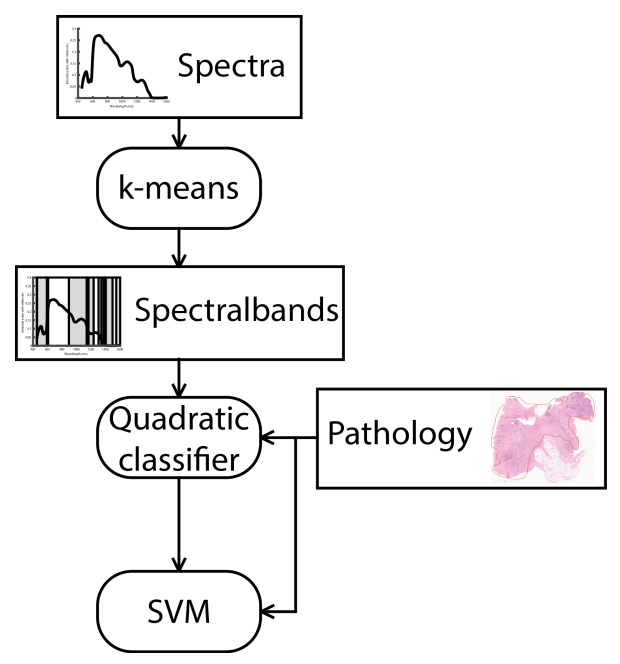

Figure 2.3: Data analysis workflow.SVM; support vector machine.

\subsection{Results}

\subsubsection{Patients}

Specimens were obtained from 38 patients: 22 male and 16 female, with a median age of 65 years (interquartile range 59-73). Most of the tumors were located in the sigmoid (17 patients), followed by the ascending and descending colon (14 and 5 patients respectively) and rectum (2 patients). Of the included tumors, 2 were adenomas and the remainder was carcinomas of which 3 were mucinous carcinomas and the rest conventional adenocarcinomas. The pT stage of 36 carcinomas was as follows; 2 were pT1, 5 were pT2, 20 were pT3 and 9 were pT4.

\subsubsection{Data}

In total 595 locations were measured, of which 147 were excluded based on the microscopic assessment. Of these, 111 locations were excluded because they were not present in the pathology slides, or the measured tissue type was not of interest. The other 36 locations were analyzed separately during a second analysis, because they were located at the border between two tissue types.

Measurements made on the mucinous carcinomas and adenomas were excluded from the data set, because the number of measurements done in these tissue 
types was too low to create a separate group.

After exclusion, 448 locations remained with a clear-cut microscopic tissue. From these locations, 134 were located in fat tissue, 150 in healthy colorectal wall and the remaining 164 in tumor tissue.

\subsubsection{Training of the classifier}

After randomly selecting $80 \%$ of the patients, the train set consisted of 30 patients and 373 measured locations. Based on the spectra from the train set, 16 spectral bands were selected (Fig. 2.4). The quadratic classifier, applied on the train data, could distinguish fat from tumor tissue and healthy colorectal wall with a mean accuracy of 1.00 (Table 2.1).

Table 2.1: Results of quadratic classifier on train dataset.

\begin{tabular}{lllll}
\hline & & \multicolumn{3}{c}{ Classification outcome } \\
& & Fat & Other & Totals \\
\hline \multirow{3}{*}{ Histology } & Fat & $112^{a}$ & 0 & 112 \\
& Other & 1 & $260^{b}$ & 261 \\
& Totals & 113 & 260 & $373^{c}$ \\
\hline
\end{tabular}

$$
\begin{aligned}
& { }^{a} \text { specificity }=1.00 . \\
& { }^{b} \text { sensitivity }=1.00 . \\
& { }^{c} \text { accuracy }=1.00 .
\end{aligned}
$$

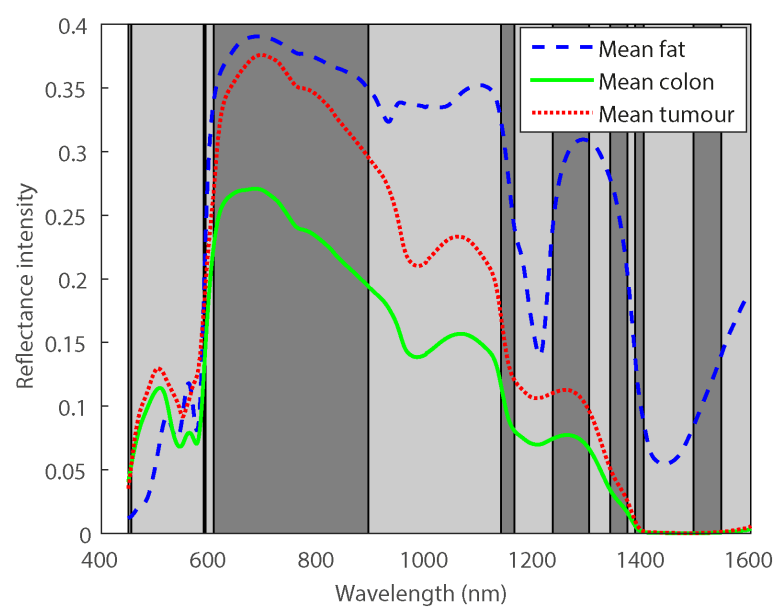

Figure 2.4: Spectral bands selected on the train set.

The ROC curve used for the optimization of the SVM is shown in Fig. 2.5. The 
accuracy of the SVM on the train data was 0.92 (Table 2.2).

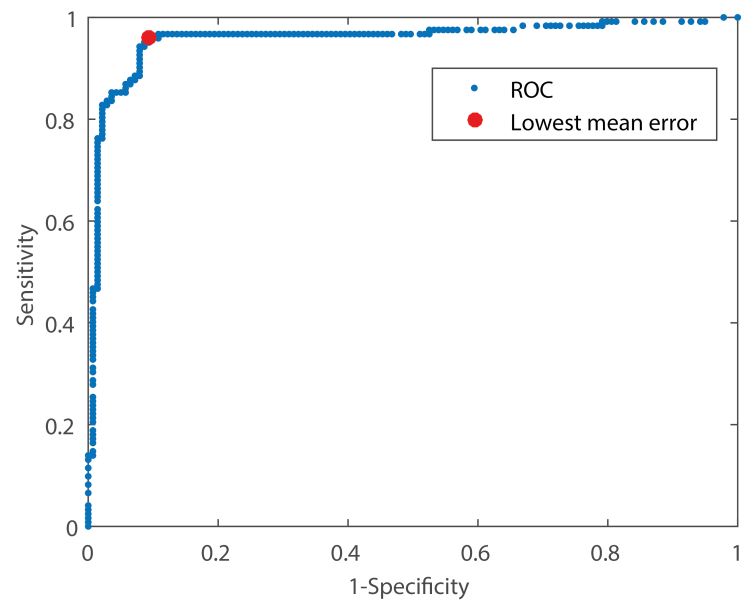

Figure 2.5: Receiver operating curve (ROC) of the SVM (support vector machine).

Table 2.2: Results of SVM (support vector machine) on train dataset.

\begin{tabular}{lllll}
\hline & & \multicolumn{3}{l}{ Classification outcome } \\
& & Colorectal wall & Tumor & Totals \\
\hline \multirow{3}{*}{ Histology } & Colorectal wall & $115^{a}$ & 6 & 121 \\
& Tumor & 14 & $125^{b}$ & 139 \\
& Totals & 129 & 131 & $260^{c}$ \\
\hline
\end{tabular}

${ }^{a}$ specificity $=0.95$.

${ }^{b}$ sensitivity $=0.90$

${ }^{c}$ accuracy $=0.92$.

Once the quadratic classifier and SVM were combined and applied to the train data, a mean accuracy of 0.95 was obtained.

\subsubsection{Performance of the classifier on the test set}

The test set consisted of $20 \%$ of the patients $(n=8)$ and a total of 75 measurement locations. The classifier pipeline applied to the test set provided a mean accuracy of 0.95 in discriminating tumor tissue from fat and healthy colorectal wall. The results from the ten iterations in the training and testing of the classifiers are shown in Table 2.3. 
Table 2.3: Results of ten time iterations of the creation and testing of the classifier. Mean and standard deviations of the accuracy per tissue type obtained on the test set, over ten iterations are shown. Finally, the mean across all tissue types is shown.

\begin{tabular}{ccccc}
\hline & Fat & Colorectal wall & Tumor & Mean across all tissues \\
\hline Mean & 1.00 & 0.93 & 0.92 & 0.95 \\
STD & 0.00 & 0.05 & 0.09 & 0.03 \\
\hline
\end{tabular}

\subsubsection{Performance classifiers on measurements of border lo- cations}

The classifier trained on the 448 locations with a clear-cut microscopic tissue, was applied to the 36 measurements that were located at the border of two tissue types. The classification of $29(80 \%)$ of these locations was in accordance with the most prominent tissue type observed in these border locations.

\subsection{Discussion}

In this ex-vivo set-up, colorectal tumor tissue can be distinguished from healthy bowel wall and surrounding fat tissue, through the use of DRS, with a mean accuracy of $0.95( \pm 0.03)$. The distinction of fat from the other two tissue types is the most accurate distinction (accuracy of 1.00).

The results obtained in this study are similar to the results reported by Schols et al. and Langhout et al. $[17,18]$. The first was able to distinguish tumor tissue from the surrounding tissue including mesenteric fat and colon tissue with an accuracy of 0.99 . However, no more than 60 tumor measurements obtained from only 6 patients were included. This limited dataset will increase the risk of overfitting. Moreover, the gold standard used by Schols et al. is the classification achieved by visual assessment by the surgeon. However, the final classification of the CRM requires histopathological examination. Therefore, it would be more accurate to use the histopathological classification as the gold standard and not the visual classification as assessed by the surgeon.

In the study by our own group reported by Langhout et al. an analytical model was used in which knowledge about the spectra of various chromophores is used to fit a curve to the obtained spectra. With the use of this knowledge, the assumption is made that the measured tissue is homogeneous. The colorectal wall exists of several layers of different tissues such as muscular and connective tis- 
sue. Therefore, the measured tissue is far from homogeneous and the analytical model cannot be applied without any reservation. In the current study no a priori knowledge about the homogeneity of the tissue was made. Moreover, compared to the study reported by Langhout et al., the current study protocol was changed in such a way that the correlation of the measurement locations with the histology was better documented. With the use of the RGB images made of every measurement, the spectra could be linked accurately to the histology annotated on the pathology slides. Furthermore, the design of the probe used in the current study is closer to a surgical tool compared to the needle used in the study reported by Langhout et al. Finally, the results obtained in the current study are as good as the results reported by Langhout et al., but were obtained without the use of fluorescence data. This does not only decrease the number of measurements needed to obtain a high accuracy, but it also reduces the costs by essentially simplifying the instrumentation and reduces the time needed for the measurements as the fluorescence signal is weak and requires a much longer integration time (seconds) than the DRS measurements.

To classify all measurements, two classifiers were used in the current study. First, the most prominent spectrum of fat was differentiated from healthy colorectal wall and from tumor tissue, both being classified into the same class. Second, to separate healthy colorectal wall from tumor measurements a more complex classifier was needed since the differences in the spectra of both tissue types are subtle (Fig. 4). Because classification was reduced to a binary task, a linear SVM was used to separate both tissue classes from each other. For well-defined tissue classes this approach resulted in an overall accuracy of $95 \%$.

In the current study, 36 locations, which were located at the border between two tissue types, were classified as well. With an accuracy of $80 \%$, these locations were classified as the tissue type that was most prominently present in the measured volume as judged histologically. It should be noted that the histological classification of the border locations may be less accurate than the histological classification of the locations containing a single tissue type. Small inaccuracies in matching the RGB images to histopathology may evidently affect inhomogeneous measurement volumes more than homogenous measurement volumes. During surgery there is a fair chance to obtain such border measurements. In fact, the entire measurement procedure is intended to assist the surgeon in assessing such locations. Therefore, these locations at the border of two tissue types are of great importance to the further development of an in vivo application. 
In addition, the absolute minimum volume of tumor tissue that can be measured should be further determined. With the current measurement technique, in which we measured a volume of about $2 \mathrm{~mm}^{3}$, it was not possible to determine a lowest detection level for small amounts of tumor tissue.

The main limitation of this study is the generalizability of the results. Neither the quadratic classifier nor the SVM provides the wavelengths or tissue constituents on which the distinction is based between tumor tissue and the healthy surrounding tissues. Therefore, from our model it is not known on what features fat can be distinguished from tumor tissue and healthy colorectal wall, and on what basis healthy colorectal wall can be distinguished from tumor tissue. As a result, it is not possible to generalize the results obtained in this study to an in vivo setting or to a differently designed measurement set-up. In the transition to the in vivo setting, there will be many changes that might influence the optical spectra. For example blood, as may be present during surgery, may have a major influence on the obtained spectrum. Still, as shown by Spliethoff et al. the influence of blood in lung biopsies may, be minimal depending on the attribution of the different wavelengths to the classification [21]. The spectral bands used in the present study included the entire blood absorption band $(450-600 \mathrm{~nm})$ in one spectral band. As shown in Fig. 4 the intensities of the three mean spectra do not differ much in this spectral band. This suggests that the absorption of blood may not have a major influence in the present classification.

The fiber distance used in this study was $2 \mathrm{~mm}$; this implies that the measurements were made at a depth of about $2 \mathrm{~mm}$ in the tissue. To be useful in colorectal surgery a sampling depth of about $5 \mathrm{~mm}$ is required. To increase the sampling depth of the DRS measurements, the fiber distance needs to be increased. However, with an increase in fiber distance the entire sampling volume increases. This results in less sensitivity of the DRS measurements to small amounts of for instance tumor. Therefore, a new study needs to be conducted to determine if the DRS measurements are still able to detect tumor at $5 \mathrm{~mm}$ depth using increased fiber distance.

In the current study, fibrosis (connective tissue without tumor cells) was not included in the measured tissue types, which is another limitation of this study. Patients with advanced stage rectal cancer almost always receive neo-adjuvant chemo-radiotherapy. These patients are highly likely to develop fibrosis on the radiation site. Fibrosis is challenging to distinguish from tumor tissue through the surgeon's visual assessment alone. Therefore, it would be beneficial to include 
this tissue type when real-time tissue classification is done. In this study, fibrosis was not included because of the limited occurrence of fibrosis in the examined specimens.

For future research two main challenges need to be addressed. First of all, fibrosis needs to be added to the tissue types that are examined. Therefore, an additional study needs to be performed that concentrates on rectal specimens of patients who underwent neoadjuvant chemo- and radiotherapy. Second, the advance to an in vivo setting needs to be made. The results of an in vivo study will be closer to the ultimate application of the real-time tissue assessment. Moreover, an in vivo study will give more insight into the workflow during surgery, which will provide a basis for the design and use of a final device used for real-time tissue assessment. The challenges of an in vivo study are first of all the identification of tumor tissue based on the visual assessment of the surgeon. Furthermore, the correlation with pathology will be challenging in an in vivo setting.

\subsection{Conclusion}

In this ex vivo study, the distinction between tumor tissue and healthy surrounding tissue in patients with colorectal cancer can be made with a high accuracy through the use of DRS measurements. The mean accuracy of the classification is 0.95 ( \pm 0.03 ). However, the results of this study are still limited to the current study set-up and protocol. Future research must be focused on the translation to the in vivo setting. This should ultimately result in reliable real-time tissue assessment leading to the increase of radical resections and decrease of postoperative morbidity rates after colorectal cancer surgery. 


\section{References}

[1] J. Ferlay et al., "Cancer incidence and mortality worldwide: Sources, methods and major patterns in GLOBOCAN 2012," International Journal of Cancer, vol. 136, no. 5, pp. E359-E386, 2015.

[2] American Cancer Society, "Cancer Facts \& Figures 2015," Cancer Facts \& Figures 2015, pp. 1-9, 2015.

[3] L. Rabeneck, S. Horton, A. G. Zauber, and C. Earle, "Cancer: Disease Control Priorities," in Disease Control Priorities 3rd Edition, ch. 6, pp. 101-119, 2015.

[4] D. M. Krishnamurty and P. E. Wise, "Importance of surgical margins in rectal cancer," Journal of Surgical Oncology, vol. 113, no. 3, pp. 323-332, 2016.

[5] I. D. Nagtegaal and P. Quirke, "What Is the Role for the Circumferential Margin in the Modern Treatment of Rectal Cancer?," Journal of Clinical Oncology, vol. 26, no. 2, pp. 303-312, 2008.

[6] M. M. Lange and C. J. H. van de Velde, "Long-term anorectal and urogenital dysfunction after rectal cancer treatment," Seminars in Colon and Rectal Surgery, vol. 21, no. 2, pp. 87-94, 2010.

[7] S. E. Delacroix and J. C. Winters, "Voiding Dysfunction after Pelvic Colorectal Surgery," Clinics in Colon and Rectal Surgery, vol. 23, no. 2, pp. 119-127, 2010.

[8] D. J. Evers, B. H. W. Hendriks, G. W. Lucassen, and T. J. M. Ruers, "Optical spectroscopy: current advances and future applications in cancer diagnostics and therapy," Future Oncology, vol. 8, no. 3, pp. 307-320, 2012.

[9] J. W. Spliethoff et al., "Monitoring of tumor radio frequency ablation using derivative spectroscopy," Journal of Biomedical Optics, vol. 19, no. 9, p. 97004, 2014.

[10] J. W. Spliethoff et al., "Improved identification of peripheral lung tumors by using diffuse reflectance and fluorescence spectroscopy," Lung Cancer, vol. 80, no. 2, pp. 165-171, 2013.

[11] L. L. Boer et al., "Fat/water ratios measured with diffuse reflectance spectroscopy to detect breast tumor boundaries," Breast Cancer Research and Treatment, vol. 152, no. 3, pp. 509-518, 2015.

[12] M. S. Bergholt et al., "Simultaneous fingerprint and high-wavenumber fiber-optic Raman spectroscopy enhances real-time in vivo diagnosis of adenomatous polyps during colonoscopy," Journal of Biophotonics, vol. 9, no. 4, pp. 333-342, 2016.

[13] H.-W. Wang et al., "Diffuse reflectance spectroscopy detects increased hemoglobin concentration and decreased oxygenation during colon carcinogenesis from normal to malignant tumors.," Optics express, vol. 17, no. 4, pp. 2805-17, 2009.

[14] G. Zonios et al., "Diffuse reflectance spectroscopy of human adenomatous colon polyps in vivo," Applied optics, vol. 38, no. 31, pp. 6628-6637, 1999.

[15] X. Shao, W. Zheng, and Z. Huang, "In vivo diagnosis of colonic precancer and cancer using near-infrared autofluorescence spectroscopy and biochemical modeling.," Journal of biomedical optics, vol. 16, no. 6, p. 067005, 2011. 
[16] H. K. Roy et al., "Spectroscopic Microvascular Blood Detection From the Endoscopically Normal Colonic Mucosa: Biomarker for Neoplasia Risk," Gastroenterology, vol. 135, no. 4, pp. 10691078, 2008.

[17] G. C. Langhout et al., "Differentiation of healthy and malignant tissue in colon cancer patients using optical spectroscopy: A tool for image-guided surgery," Lasers in Surgery and Medicine, vol. 47, no. 7, pp. 559-565, 2015.

[18] R. M. Schols, P. Dunias, F. P. Wieringa, and L. P. S. Stassen, "Multispectral characterization of tissues encountered during laparoscopic colorectal surgery," Medical Engineering and Physics, vol. 35, no. 7, pp. 1044-1050, 2013.

[19] R. Nachabé et al., "Estimation of biological chromophores using diffuse optical spectroscopy: benefit of extending the UV-VIS wavelength range to include 1000 to $1600 \mathrm{~nm}$," Biomedical Optics Express, vol. 1, no. 5, pp. 1432-1442, 2010.

[20] R. Nachabe et al., "Estimation of lipid and water concentrations in scattering media with diffuse optical spectroscopy from 900 to 1600 nm," vol. 15, pp. 37010-37015, 2010.

[21] J. W. Spliethoff et al., "Real-time in vivo tissue characterization with diffuse reflectance spectroscopy during transthoracic lung biopsy: a clinical feasibility study," Clinical Cancer Research, vol. 22, no. 2, pp. 357-365, 2016 


\section{Chapter 3}

\section{Hyperspectral imaging for tissue classification, a way towards smart laparoscopic colorectal surgery}

Elisabeth J.M. Baltussen, Esther N.D. Kok, Susan G. Brouwer de Koning, Joyce Sanders, Arend G.J. Aalbers, Niels F.M. Kok, Geerard L. Beets, Claudie C. Flohil, Sjoerd C. Bruin, Koert F.D. Kuhlmann, Henricus J.C.M. Sterenborg, Theo J.M. Ruers, Journal of Biomedical Optics, 2019, Volume 24 (1) 


\section{Abstract}

In the last decades laparoscopic surgery has become the gold standard in patients with colorectal cancer. To overcome the drawback of reduced tactile feedback, real-time tissue classification could be of great benefit. In this ex vivo study, hyperspectral imaging was used to distinguish tumor tissue from healthy surrounding tissue. A sample of fat, healthy colorectal wall and tumor tissue was collected per patient and imaged using two hyperspectral cameras, covering the wavelength range from $400-1700 \mathrm{~nm}$. The data was randomly divided into a train $(75 \%)$ and test $(25 \%)$ set. After feature reduction, a quadratic classifier and support vector machine were used to distinguish the three tissue types. Tissue samples of 32 patients were imaged using both hyperspectral cameras. The accuracy to distinguish the three tissue types using both hyperspectral cameras was 0.88 $(S T D=0.13)$ on the test dataset. When the accuracy was determined per patient, a mean accuracy of $0.93(S T D=0.12$ ) was obtained on the test dataset. This study shows the potential of using hyperspectral imaging in colorectal cancer surgery for real-time tissue classification which could improve clinical outcome. Future research should be focused on imaging entire colon/rectum specimen and the translation of the technique to an intra-operative setting. 


\subsection{Background}

Colorectal cancer is the third most commonly diagnosed cancer worldwide and the fourth cause of death due to cancer [1,2]. For patients with colorectal cancer, surgery is the cornerstone of the treatment. In the last decades, laparoscopic surgery for colorectal cancer has become common practice. Randomized controlled trials have proven similar clinical outcomes for laparoscopic surgery as for open surgery with a decrease in hospital stay [3]. One of the drawbacks of laparoscopic surgery is the reduced tactile feedback during surgery $[4,5]$. The lack of tactile feedback makes tissue recognition more cumbersome, especially in areas where radical resection margins are often compromised like in locally advanced tumors and in rectal cancer. Hence, an alternative technique that would enable the surgeon to distinguish tumor from normal tissue during laparoscopic surgery in real-time would be of great benefit to secure radical resection in difficult areas, such as in rectal cancer. We will investigate the use of hyperspectral imaging (HSI) as a tool to ensure radical margins in these circumstances, distinguishing tumor from healthy colorectal tissue.

In $\mathrm{HSI}$ a broadband light source is used to illuminate an object, like e.g. tissue. The light will interact with the tissue by reflection, scattering and absorption of the photons. This interaction strongly depends on the tissue type and wavelength [6]. After several interactions within the tissue, part of the light will be reflected to the surface of the tissue and is detected by the hyperspectral camera. In the resulting hyperspectral image the tissue specific spectral changes of the light can be analyzed. Ultimately, hyperspectral imaging result in 2D images of the object obtained over several wavelengths, resulting in a 3D datacube with 2 spatial dimensions and in the third dimension the wavelengths (Fig. 3.1).

Previous studies used HSI as a diagnostic tool in cancer of the cervix [7], breast [8], skin [9], tongue [10], head and neck [11, 12], gastric [13, 14], colon and rectum [15-22]. In colorectal cancer most studies focused on hyperspectral imaging of the hematoxylin-eosin (H\&E) pathology slides [15-19] or tissue classification during endoscopy to distinguish tumor from healthy tissue [20-22]. In the current ex vivo study we investigated the use of HSI to differentiate normal colorectal tissue from tumor tissue in a surgical setting looking from the surface of the tissue instead of from the lumen of the colon. To this end colorectal cancer samples obtained during surgery were imaged using HSI in the visible to near-infrared region. The spectra obtained from these images were classified using a classifi- 

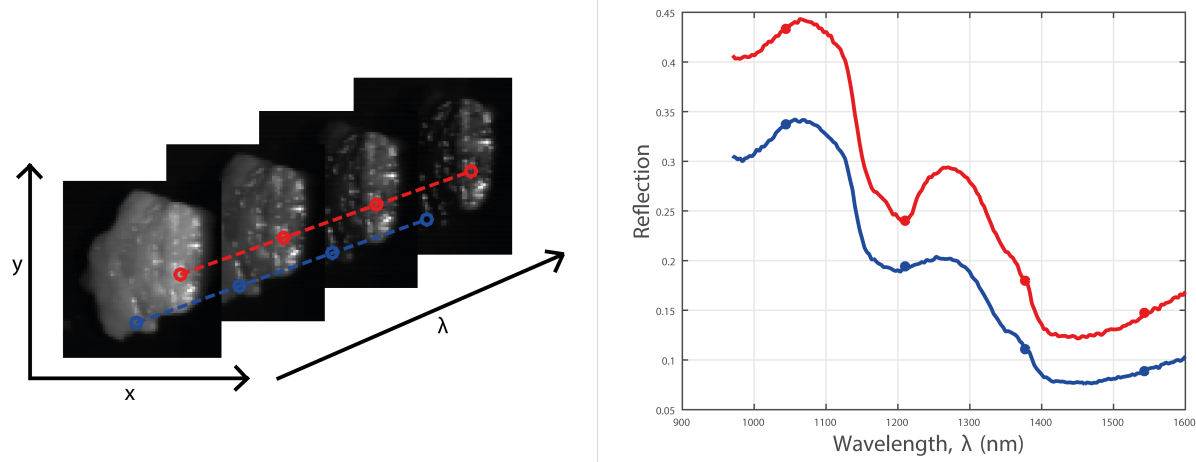

Figure 3.1: Hyperspectral image, with two spatial dimensions $(x, y)$ and one spectral dimension $(\lambda)$. On the right side the spectra of the two selected pixels are shown.

cation algorithm and were verified with histology. Finally, complete hyperspectral images were classified using the trained classifier. The ultimate goal is to develop a real-time technique for tissue identification in laparoscopic colorectal surgery.

\subsection{Materials and methods}

\subsubsection{Hyperspectral cameras}

Two hyperspectral cameras were used for the measurements, one in the visual wavelength range and one in the near-infrared wavelength range. The first was a SPECIM (Spectral Imaging Ltd., Finland) spectral camera (PFD-CL-65-V10E) with a wavelength range from $400 \mathrm{~nm}$ to $1000 \mathrm{~nm}$, a CMOS sensor of 1 by 1312 pixels and a spectral resolution of $3.0 \mathrm{~nm}$, hereafter referred to as the visual camera. The second was also a SPECIM spectral camera (VLNIR CL-350-N17E) with a wavelength range from $900-1700 \mathrm{~nm}$, an InGaAs sensor of 1 by 320 pixels and a spectral resolution of $5.0 \mathrm{~nm}$, hereafter referred to as the near-infrared camera. Both cameras were push broom cameras, meaning that they image a single line (x-axis) only. To obtain a full 2D image the samples were placed on a translational stage and pushed underneath the camera (y-axis). All samples were illuminated using a halogen light source.

Both, a dark and white reference image were taken before each measurement. The dark reference image was taken by closing the shutter of the cameras. The white reference image was taken on a Spectralon reflectance standard. The linear 
behavior of the visual camera allowed for a simple calibration of the camera using Equation 3.1. In Equation $3.1 x_{c a l}$ is the calibrated spectrum, $x$ is the original spectrum, $D_{\text {ref }}$ is the dark reference and $W_{\text {ref }}$ is the white reference. The nearinfrared camera, however, had a slight non-linear behavior and was therefore calibrated using a fourth order polynomial, Equation 3.2, instead of the linear formula [23]. In Equation 3.2, $b_{i}$ are variables determined using a series of 5 reference samples. The values of $b_{i}$ differ per pixel and wavelength. Furthermore, $x$ is the original spectrum and $x_{c a l}$ is the calibrated spectrum [24]:

$$
\begin{gathered}
x_{c a l}=\frac{x-D_{r e f}}{W_{r e f}-D_{r e f}} \\
x_{c a l}=b_{0}+b_{1} x+b_{2} x^{2}+b_{3} x^{3}
\end{gathered}
$$

\subsubsection{Study protocol}

Patients who underwent surgery for colorectal cancer in the Antoni van Leeuwenhoek - The Netherlands Cancer Institute (Amsterdam, the Netherlands) and the Slotervaart Medical Centre (Amsterdam, the Netherlands) were included in this ex vivo study. The study was performed under approval of the protocol by the institutional ethics review board.

Immediately after colorectal resection, the entire resected specimen was taken to the pathology department. Cross sections were cut by the pathologist from the specimen and three tissue samples were obtained; tumor tissue, healthy colon or rectal wall and (peri-colorectal) fat. The cross sections were placed in a pathology cassette where they remained during the entire data acquisition. All measurements were performed within one hour after specimen resection. Before the hyperspectral measurements, an RGB image was taken of each tissue sample. Next, hyperspectral images were obtained from the tissue samples after which the samples were taken back to the pathology department. The samples were processed according to standard protocol in the same pathology cassette, to prevent large tissue deformations. The corresponding H\&E slides were examined by the pathologist, who annotated the various tissue types. For further data analysis the digitized annotated slides were registered to the RGB image and the RGB image to the $\mathrm{HSI}$ in Matlab (version 8.5, MathWorks Inc., Natick, Massachusetts, United States), using a non-rigid transformation to overcome the effects of mechanical deformation of the tissue during the standard work flow of tissue processing and 
staining. Finally the registered pathology slide was registered to the registered RGB image (Fig. 3.2). Using these registrations, each pixel from the hyperspectral image could be given a histological classification. To create a database of hyperspectral pixels, pixels were manually selected within areas that were defined by the pathologist as absolute certain for a tissue type. About 30 pixels per tissue type per patient were selected when possible. When the surface area of a tissue type was too small to select 30 individual pixels, less pixels were selected. Pixels in the mucosal layer were not taken into account because the mucosa will not be visible during the ultimate surgical application of the technology. Hence, the pixels from the healthy colorectal wall were all in the muscular layer.

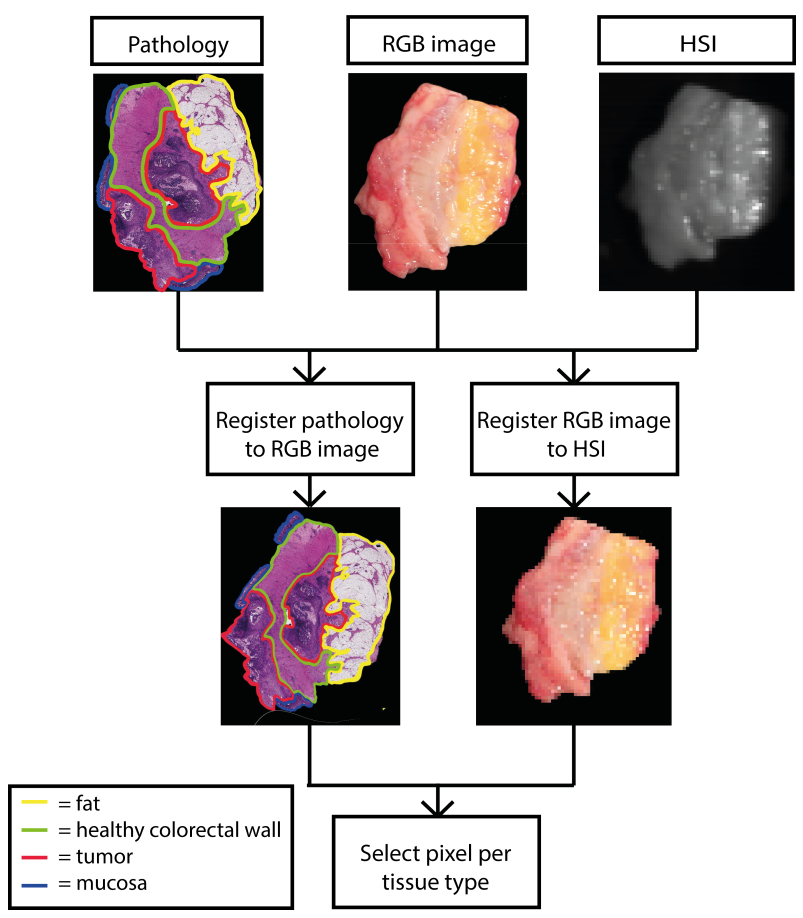

Figure 3.2: Registration of the HSI, RGB and pathology images. In the upper row from left to right, annotated pathology image (yellow = fat, green = healthy colorectal wall, red = tumor, blue = mucosa), RGB image and HSI. The second row from left to right, the annotated pathology image registered to the RGB image and the RGB image registered to the HSI. 


\subsubsection{Data preprocessing}

Preprocessing of the data was performed using a $3.40 \mathrm{GHz}$ Intel Xeon E3-1240 CPU processor and 16 GB RAM and consisted of two steps. First the spectra were normalized using standard normal variate (SNV) normalization [25]. SNV normalization was performed for each individual spectrum. First the mean was subtracted from the spectrum after which the spectrum was divided by the standard deviation of the spectrum, see Equation 3.3. Here $x_{c o r r}$ is the normalized spectrum, $x_{c a l}$ is the calibrated spectrum as given in Equation 3.1 or 3.2 and $x_{\text {mean }}$ and $x_{s t d}$ are the mean and standard deviation of $x_{c a l}$ respectively. This normalization created a zero baseline and a variance equal to one for all spectra.

$$
x_{c o r r}=\frac{x_{c a l}-x_{\text {mean }}}{x_{s t d}}
$$

After combination of the visual and near-infrared images, all outliers caused by specular reflection were removed. Outliers were defined as spectra with an average distance from the mean spectrum of more than three standard deviations, determined over all wavelengths.

Next, in order to combine the spectra of the two cameras, the visual images were down sampled. Down sampling was necessary because of the higher resolution of the visual camera compared to the near-infrared camera. A rigid spatial registration was performed obtaining a pixel to pixel correlation between the images of the two cameras.

\subsubsection{Data analysis}

Data analysis was performed using the PerClass toolbox (Academic version 5.0, PR Sys design, Delft, the Netherlands) in Matlab. The data were randomly divided into a train and test set. Per patient all spectra were assigned to either the train or test set, indicating that spectra from one patient were not split between the train and test set. The train set contained $75 \%$ of the patients and the remaining $25 \%$ was used as a test set. The data contained a hyperspectral image from both the visual and near-infrared camera.

The development of the classification algorithm consisted of 3 steps. First, feature reduction was applied to the spectra to prevent overfitting of the classifier. For this purpose k-means clustering was used to determine spectral bands. The clustering is based on the average intensity values of the spectra from the train dataset. 
For each cluster, if the wavelengths are not continues the cluster will be divided into multiple spectral bands. The spectral bands were determined once on the train set and were also used on the test set. From these spectral bands only the mean intensity was used as a feature in the classification algorithm. Second, fat was classified first using a quadratic classifier, which was optimized with a tenfold cross-validation. The selected optimum in the ROC curve was the point with the lowest mean error. Third, a linear support vector machine (SVM) was used to distinguish the tumor spectra from the healthy colorectal wall spectra. The SVM was also optimized using a ten-fold cross-validation. Classification of the pixels was done based on the probability given by the classifiers. Pixels were assigned to the tissue type with the highest probability. The performance of the classifiers was compared using the area under the ROC curve (AUC), the accuracy, the sensitivity and specificity and the Matthews Correlation Coefficient (MCC) (Equation 3.4).

$$
M C C=\frac{T P \times T N-F P \times F N}{\sqrt{(T P+F P)(T P+F N)(T N+F P)(T N+F N)}}
$$

In Equation 3.4, TP, TN, FP and FN are the number of true positives, true negatives, false positives and false negatives, respectively. The MCC returns a value from -1 to +1 where -1 indicates a total disagreement and +1 a perfect prediction. The accuracy was determined in two different ways. The first accuracy was determined per tissue type and thereafter averaged. The second accuracy was determined per patient and averaged.

Finally, to assess the contribution of each camera, the classification was also trained and tested on datasets containing data from only one of the two cameras. The performance of this classification was compared with the classification of the dataset containing data from both cameras, using the ROC curves and the performance measures.

\subsection{Results}

\subsubsection{Patients}

In total 54 patients were included in this study: 27 men (50\%), 27 women (50\%), with a median age of 65.5 years (IQR: $60-73$ ). The samples of 32 patients were imaged with both the near-infrared and the visual camera, 22 additional patients 
were imaged with only the near-infrared camera. Most of the tumors were located in the colon and sigmoid. One sample showed complete pathological response on pre-operative treatment and thus, no tumor tissue could be taken from the sample. Patient and tumor characteristics are described in Table 3.1.

Table 3.1: Characteristics of the group of patients measured with the near-infrared camera and the group of patients measured with the visual camera.

\begin{tabular}{llcc}
\hline \multirow{2}{*}{ Total number of patients } & & NIR camera & VIS camera \\
\hline \multirow{2}{*}{ Gender } & Male & 54 & $32^{*}$ \\
\hline \multirow{3}{*}{ Age } & Female & 27 & 14 \\
& Median & 27 & 18 \\
\hline \multirow{4}{*}{ Tumor location } & Interquartile range & 65.5 & 66.5 \\
& Cecum & $60-73$ & $60-71.5$ \\
\hline \multirow{4}{*}{ Tumor type } & Colon & 7 & 7 \\
& Sigmoid & 23 & 12 \\
& Rectum & 21 & 11 \\
\multirow{4}{*}{ Tumor stage } & Complete response & 3 & 2 \\
& Adenocarcinoma & 1 & 1 \\
& Mucinous adenocarcinoma & 76 & 25 \\
& pT0 & 1 & 6 \\
\hline & pT1 & 3 & 1 \\
& pT2 & 9 & 4 \\
& pT3 & 26 & 16 \\
& pT4 & 15 & 10 \\
\hline
\end{tabular}

*All patients measured with the VIS camera are also included in the NIR camera measurements

\subsubsection{Data acquisition and processing time}

The obtained tissue samples were first placed under the near-infrared camera and subsequent under the visual camera. Data acquisition times were 20 and 30 seconds for the near-infrared and the visual camera, respectively. In total, data of one patient was acquired in 1 minute. The duration of image preprocessing for each tissue sample was 60 seconds in total for both cameras combined for all wavelengths. After training of the classifier, classification of the test data took 2 seconds per patient. 


\subsubsection{Classification with the combination of visual and near- infrared camera}

For the classification of the combination of the visual and near-infrared camera, only the tissue samples scanned with both cameras were included. The dataset of the combined visual and near-infrared camera images contained 2194 spectra, from 32 patients. After outlier removal 2170 spectra were present in the combined dataset, of which 857 were taken from fat, 563 from muscle and 750 from tumor. The train set consisted of 24 patients with a total of 1726 spectra. The test set consisted of the remaining 8 patients and 444 spectra. Due to the presence of noise in the lower and upper wavelength range of both cameras, the wavelength ranges of 450-950 and 970-1600 were selected to analyze for the visual and nearinfrared camera respectively.

As shown in Figure 3.3, the reflection spectra obtained with the visual camera and the near-infrared camera are not connected after calibration. This is related to differences in optical geometry of the two camera setups. In Figure 3.3, the large difference in normalized intensity visible at $960 \mathrm{~nm}$ is caused by individual SNV normalization of both cameras before combining the datasets.

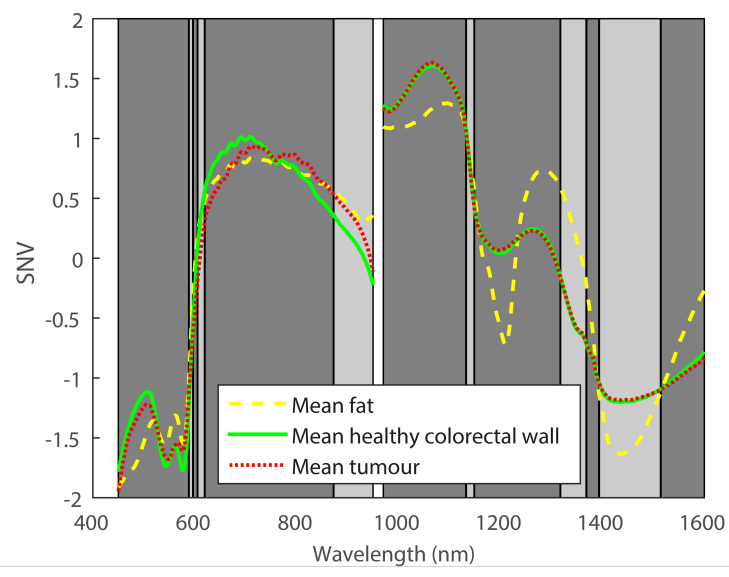

Figure 3.3: Spectral bands determined for the dataset with the combination of visual and near-infrared camera images are shown together with the mean spectra of fat (yellow), healthy colorectal wall (green) and tumor (red). The 13 spectral bands all have a different gray value and are separated by black vertical lines. Between 950 and $970 \mathrm{~nm}$ a gap is shown in the data. This region is not covered by the cameras.

Based on the train set, 13 spectral bands were determined as shown in Figure 3.3. On the train data the quadratic classifier obtained an MCC, AUC, accuracy, sen- 
sitivity and specificity of 1.00 to separate fat from healthy and tumor. The SVM applied on the train dataset, to distinguish tumor from muscle, provided an MCC of 0.83 , an AUC of 0.98 , an accuracy of 0.91 and the sensitivity and specificity were 0.93 and 0.90 , respectively. The accuracy of combination of the quadratic classifier and the SVM on the train dataset was 0.94 (STD $=0.04$ ) when assessed on the tissue types. When determined per patient and averaged, a train accuracy of $0.94($ STD $=0.13)$ was obtained.

The results of the combination of the quadratic classifier and the SVM on the test dataset are shown in Table 3.2. The accuracy determined per tissue type and averaged on the test dataset was $0.88(S T D=0.13)$. The accuracy calculated per patient and thereafter averaged was $0.93(\mathrm{STD}=0.12)$.

Table 3.2: Results of the combined classifiers on the test dataset with a combination of visual and near-infrared camera images. The mean accuracy averaged over the tissue types was 0.88 (STD = $0.13)$ and over the patients was $0.93(\mathrm{STD}=0.12)$.

\begin{tabular}{|c|c|c|c|c|c|}
\hline & & \multicolumn{4}{|c|}{ Decision based on hyperspectral data } \\
\hline & & Fat & Muscle & Tumor & Total \\
\hline \multirow{4}{*}{ Gold Standard } & Fat & 187 & 0 & 0 & 187 \\
\hline & Muscle & 30 & 81 & 4 & 115 \\
\hline & Tumor & 0 & 9 & 133 & 142 \\
\hline & Total & 217 & 90 & 137 & 444 \\
\hline
\end{tabular}

In Figure 3.4, the results of the classification of all pixels in a hyperspectral image of one patient from the test set are shown. The different colors represent different tissue types. The certainty of the classification, based on the probability, is shown by the intensity of the color. The more intense the color is, the higher the certainty of the classifier is for this classification.

\subsubsection{Classification with a single camera}

The classification of fat, healthy colon or rectal wall and tumor was also performed on the datasets including only one of the two cameras. The dataset with only spectra from the near-infrared camera contained 54 patients and 4352 spectra of which 1690 were measured in fat, 1251 in the muscular layer of healthy colon or rectal wall and 1411 in tumor. After removal of the outliers 4309 spectra remained (1676 fat, 1232 muscle, 1401 tumor). For the train set, 41 patients were randomly selected with a total of 3241 spectra. The test set contained 13 patients and 1068 spectra.

For the images of the visual camera the same pixels were used as selected for 


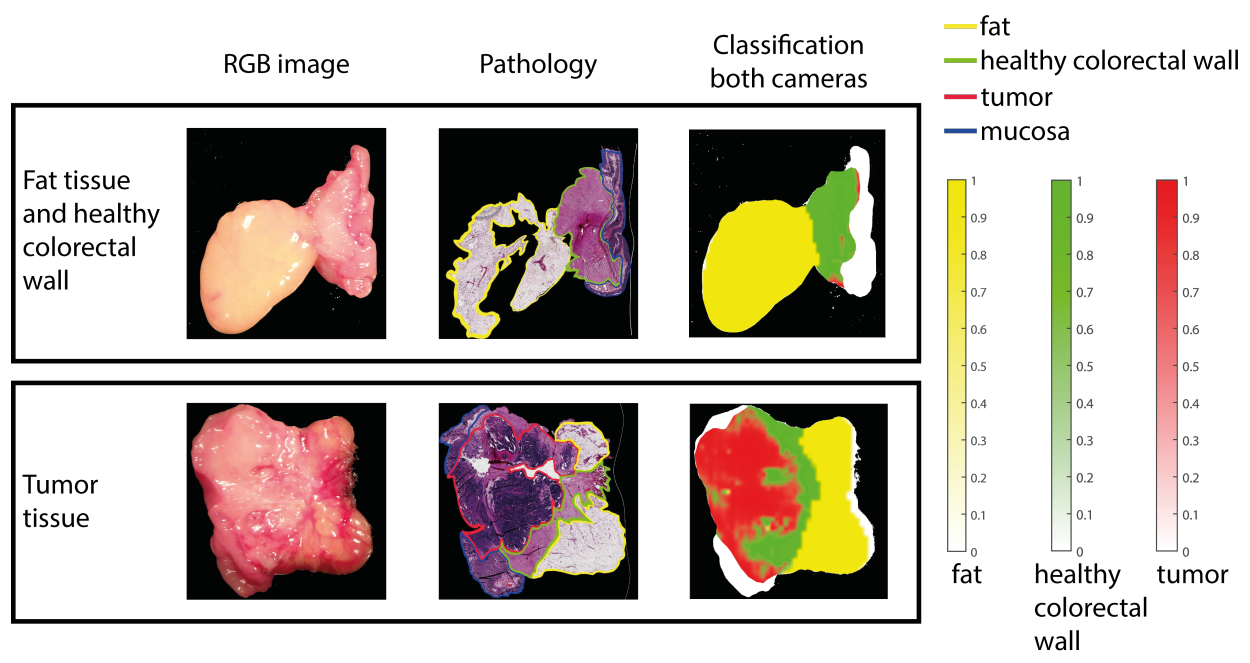

Figure 3.4: Classification of the tissue samples of one patient from the test set of the combined data set of the visual and near-infrared camera. In the first column the RGB image of each tissue sample is shown. The second column shows the registered annotated pathology image (yellow $=$ fat, green = muscle or healthy colorectal wall, red $=$ tumor, blue = mucosa). In the third column the classification based on the visual and near-infrared spectra is shown projected on the binary mask of the RGB image (yellow $=$ fat, green $=$ muscle of healthy colorectal wall, red $=$ tumor). The first row shows the healthy tissue including fat and healthy colorectal wall. The second row shows the tumor tissue sample. Tissue annotated by the pathologist as mucosa (blue) is not classified and is shown a white in the third column.

the images of the near-infrared camera. A total of 2194 spectra, from 32 patients, were included in this dataset. From the spectra, 866 were measured in fat, 569 in muscle and 759 in tumor. After removal of the outliers 2164 spectra remained (854 fat, 560 muscle and 750 tumor). The train set included 24 patients with 1723 spectra, the remaining 8 patients were included in the test set which contained 441 spectra.

After spectral bands were extracted from the datasets the quadratic classifiers were trained and ROC curves for both datasets were obtained. In Figure 3.5 the ROC curves of both classifiers are shown together with the ROC curve of the classifier created with the combined dataset. This shows a slightly worse performance for the dataset with only visual camera images compared to the dataset with only near-infrared images or the dataset including image of both cameras. This was also seen in the performance measures, with an MCC of 0.90 for the dataset with only visual camera images, and an MCC of 0.99 and 1.00 for the dataset with only near-infrared camera images and the combined dataset, respectively. All other performance measures showed the same trend. 


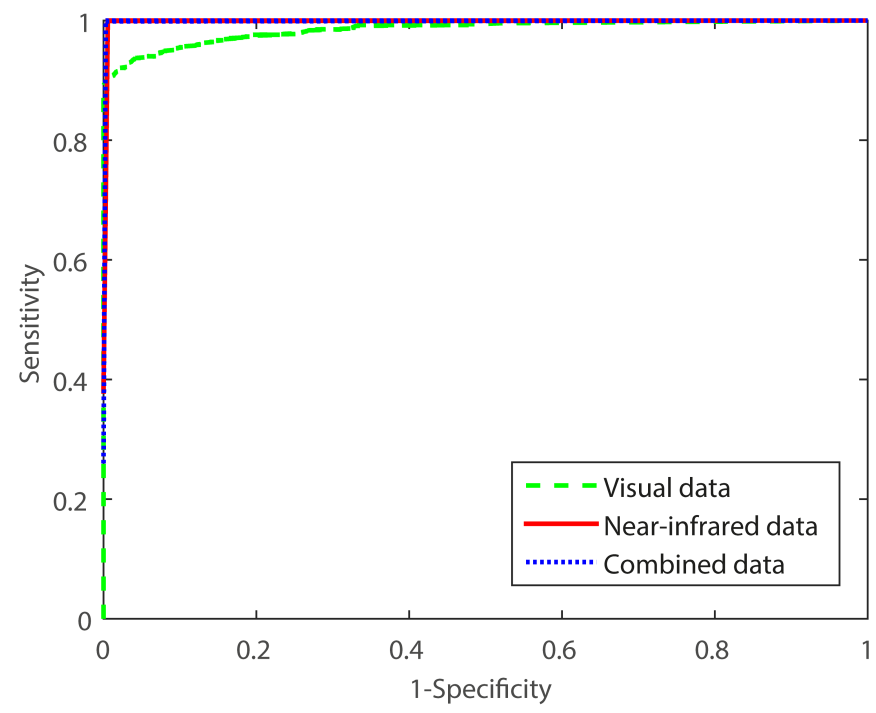

Figure 3.5: ROC curves of the train results of the quadratic classifier distinguishing fat from all other tissue types. The three datasets are shown as the visual camera (green), near-infrared camera (red) and the combination of the visual and near-infrared camera (blue).

In Figure 3.6 the ROC curves of the SVM's are shown for the three train datasets. Here again the dataset containing only visual camera images showed the worst performance. However, there is a clear difference between the dataset containing only near-infrared camera images and the dataset containing images of both cameras. Where the latter outperformed the first. A summary of the performance measures of the SVM is shown in Table 3.3. The same trend is shown for all performance of the three datasets; the dataset including only visual camera images performed worst and the combined dataset performed best.

The results of the test set of the two datasets containing data of only one of the two cameras resulted in an accuracy for determining the tissue types of 0.67 $(\mathrm{STD}=0.19)$ and $0.83(\mathrm{STD}=0.12)$ for the visual camera data and near-infrared camera data respectively. The accuracy calculated per patient and averaged was $0.71(S T D=0.19)$ for the visual camera data and $0.83(S T D=0.14)$ for the nearinfrared camera data.

The classifiers created were also used to classify the spectra from each pixel of entire hyperspectral images of one of the patients from the test set that was imaged by both cameras. In Figure 7, the result of this classification is shown for all three classifications. 


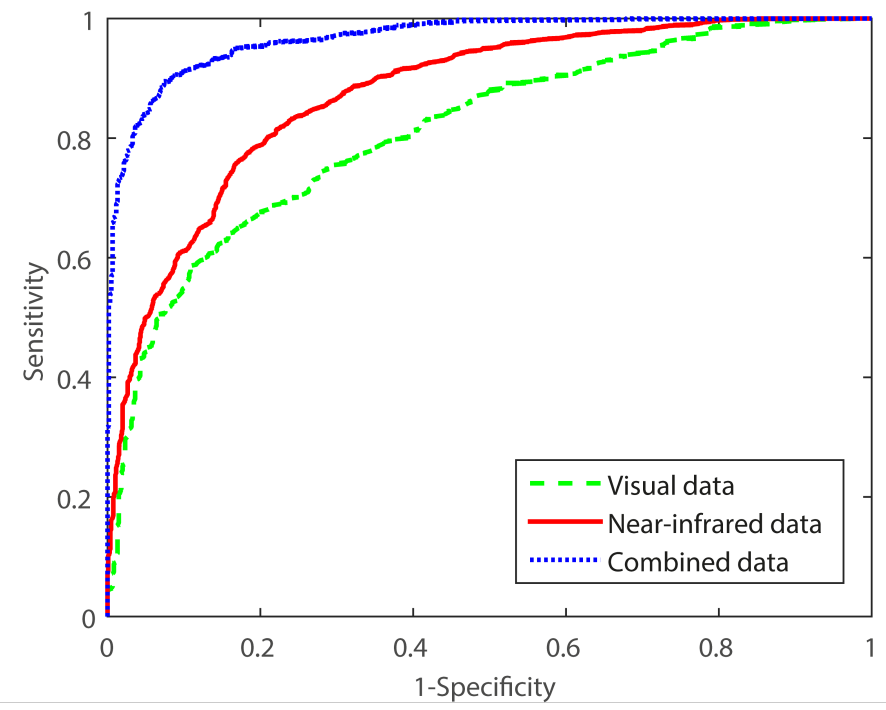

Figure 3.6: ROC curves of the train results of the SVM's distinguishing tumor from healthy colorectal tissue. The three datasets are shown as the visual camera (green), near-infrared camera (red) and the combination of the visual and near-infrared camera (blue).

Table 3.3: Performance measures for the SVM from the three train datasets.

\begin{tabular}{lccc}
\hline Performance measure & Visual camera & Near-infrared camera & Combined dataset \\
\hline MCC & 0.50 & 0.59 & 0.83 \\
AUC & 0.81 & 0.87 & 0.98 \\
Accuracy & 0.74 & 0.80 & 0.91 \\
Sensitivity & 0.77 & 0.78 & 0.93 \\
Specificity & 0.74 & 0.81 & 0.90 \\
\hline
\end{tabular}

\subsection{Discussion}

In this study the potential added value of HSI for real-time tissue classification during colorectal cancer surgery was examined. As a first step an ex vivo study was designed in which tissue samples from colorectal cancer surgery were imaged with two hyperspectral cameras. One camera obtained images in the visual wavelength range (400-1000 $\mathrm{nm}$ ), while the second camera obtained images in the near-infrared wavelength range $(900-1700 \mathrm{~nm})$. HSI allowed accurate discrimination of fat, healthy colon or rectal wall and tumor tissue, with an accuracy of $0.88(S T D=0.13)$ for the combination of the visual and near-infrared camera 


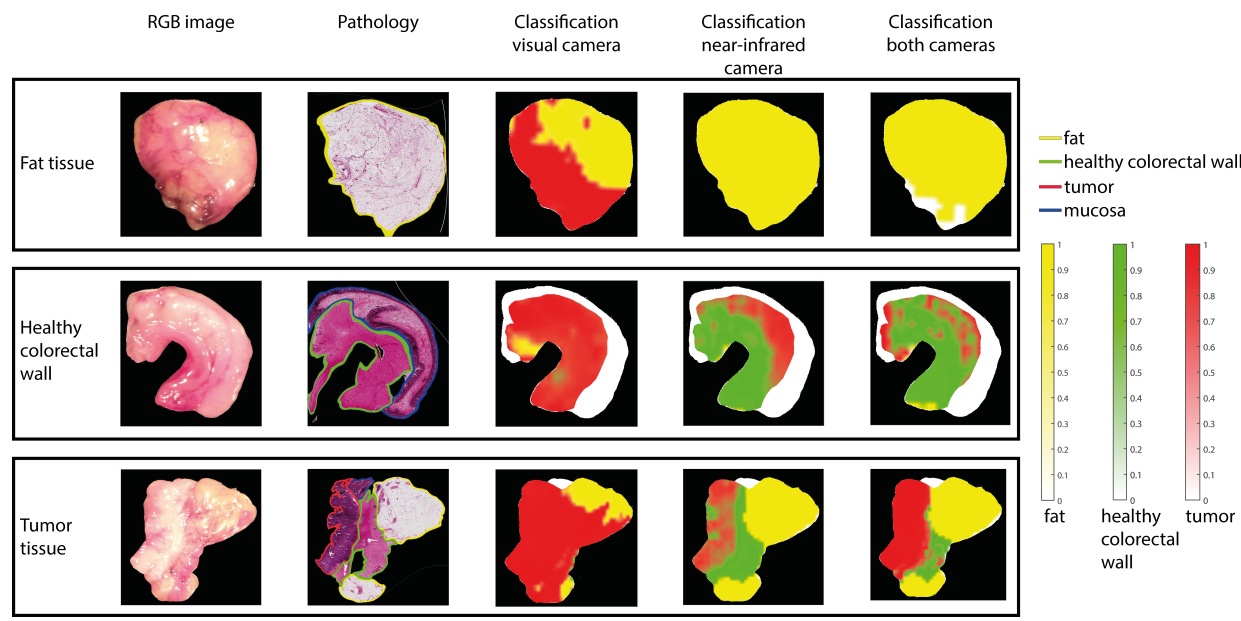

Figure 3.7: Classification of the tissue samples of one patient from the test dataset of the combined dataset. In the first column the RGB image of each tissue sample is shown. The second column shows the registered annotated pathology H\&E image (yellow $=$ fat, green = muscle or healthy colorectal wall, red $=$ tumor, blue $=$ mucosa). In the third to fifth column from the classification based on the visual image only, the classification based on the near-infrared image only and the classification based on the combined visual and near-infrared image are shown respectively, projected on the binary mask of the RGB image (yellow $=$ fat, green $=$ muscle of healthy colorectal wall, red $=$ tumor). From top to bottom fat, healthy colorectal wall and tumor tissue is shown. Tissue annotated as mucosa (blue) by the pathologist is not classified and shown as white in the third to fifth column.

images.

Current literature of hyperspectral imaging in colorectal cancer was mainly focused on the classification of H\&E pathology slides [15-19] or classification of tissue during endoscopy [20-22]. For the first application hyperspectral images were made of $\mathrm{H} \& \mathrm{E}$ pathology slides to obtain objective classification into healthy or malignant tissue of colon biopsies [15-19]. This application is far from the goal of the current study as the current study is focused on real-time imaging during surgery. For the second application, a hyperspectral camera was combined with an endoscope, to obtain hyperspectral images during endoscopy. The study by Kumashiro et al. [22] obtained in vivo hyperspectral data during colonoscopy and was able to distinguish tumor from healthy mucosa with a sensitivity of 0.73 and a specificity of 0.82 , using Pearson correlation analysis. The study by Han et al. [21] obtained better results with an accuracy of 0.94 and a sensitivity and specificity of 0.97 and 0.91 respectively. This study used hyperspectral images in the spectral range from 405 to $665 \mathrm{~nm}$, so only the visual wavelength range. These results are better than the results shown in the current study for the dataset including only the visual camera images. The main difference between the two studies 
and the current study is the location of the measurements. Han et al. [21] and Kumashiro et al. [22] performed measurements of the lumen of the colon during endoscopy, whereas the current study focused on the surgical application and performed measurements from the surface of the colon.

In a previous study, we showed the possibility to distinguish the three tissue types - fat, healthy colon or rectal wall and tumor - using fiberoptic diffuse reflectance spectroscopy (DRS) in a surgical setting [26]. The information obtained in fiberoptic DRS is very similar to the information obtained with a hyperspectral camera system. Therefore, it is not surprising that the results obtained in the current study are comparable to the results obtained in the previous study using DRS. However, the accuracy of the current study is slightly less compared to the accuracy obtained with the DRS measurements (accuracy $=0.95$, STD $=0.03$ ). An explanation for this difference might be due to the correlation with the gold standard, histology, which is less challenging for the fiberoptic point measurements performed in the previous study. In the testing of the combination of the two cameras, 30 muscle spectra of one particular patient were classified as fat. These misclassifications are most likely due to a fault in the registration between the hyperspectral images and the histology in this specific patient. The influence of this one patient can be seen in the accuracy determined per patient, which is 0.93 and similar to the accuracy obtained in the DRS study.

For the evaluation of the performance of the classifiers, six different performance measures were used. Of these parameters the MCC and AUC are most accurate in the current study, because these parameters are relatively insensitive to the effect of an imbalanced dataset [27]. However, for the combination of the quadratic classifier and the SVM the MCC and AUC cannot be used, because both measures only account for a two class problem. For the quadratic classifier no large differences are shown between the performance measures for the three datasets. However, the performance measures of the SVM did show a difference. The combination of the two cameras clearly outperforms the datasets using only one of the two cameras (Table 3.3). For the combination of the quadratic classifier and the SVM the accuracies can be compared. Here, the combination of the two cameras out performs the datasets with data from only one of the two cameras.

Comparing the two cameras, the near-infrared camera slightly outperforms the visual camera, with a MCC of 0.59 and 0.50 respectively. In Figure 3.3, only the average curves are shown per tissues type. Although the difference between the average spectra of colon and tumor tissue is bigger in the visual part of the 
spectrum, also the standard deviation (not shown) is higher in the visual part compared to the near-infrared part. Furthermore, the SVM used for the classification of healthy colon and tumor does not take into account each feature individually but uses the combination of features to find the optimal hyperplane which differentiates the two classes. In accordance, the combination of the features from the near-infrared part of the spectrum might give a better result than the combination of the features from the visual part of the spectrum. It is hard to visualize these distinctive differences in the near-infrared part of the spectrum. In line with the explanation above, as mentioned before, the MCC value for the discrimination between healthy and tumor was slightly higher in the near-infrared part of the spectrum, the combination of the near-infrared and visual part of the spectrum gives the best results because of the combination of the features that can be made. This increases the accuracy with 0.21 to 0.88 .

For the translation of the technique to an in vivo setting, where it will be used during surgery, the large dependence on the near-infrared wavelength ranges is favorable. The main difference between an ex vivo setting and an in vivo setting is the presence of blood. For oxygenated and deoxygenated blood, the main absorption bands are located in the visual wavelength range. Therefore, blood absorption will have no influence in the near-infrared wavelength range [28]. The influence of blood in the translation to an in vivo setting using a classification method based mostly on the near-infrared wavelength range will thus be small [29].

Previous work in our group showed good results in combining a quadratic classifier and a linear SVM in tissue identification in colorectal cancer using fiberoptic DRS [26]. In the current study also two different classifiers to distinguish between fat, healthy colorectal wall and tumor are used. First a quadratic classifier was used to classify fat and second a linear SVM was used to distinguish healthy colorectal wall from tumor. Because fat was first classified with the quadratic classifier, a binary task was left for the SVM. Therefore, a simple linear SVM could be used to distinguish healthy colorectal wall from tumor tissue. To perform a classification of three tissue types using only SVM's, a one-against-one or one-againstall classification should be performed. This will result in a combination of at least three SVM's, which will be a more complex classifier compared to the combination currently used. The more complex the classifier is, the more prone the classifier is to overfitting. Because the classification of fat was easy to perform, a more simple approach could be used in the form of a two-step classification, combing a quadratic classifier with a single SVM. 
The classifiers created in this study were only based on the spectral features. However, because 2D images were obtained with the hyperspectral cameras, there is also an option to use spatial and textural properties of the images to classify the pixels. In the current study this option was not used because the spatial and textural properties during surgery will be very different compared to the properties which would have been obtained in this study. However, for future studies textural properties may be taken into account and could be used to further improve the current classification results.

In this ex-vivo study, the pathologist cut cross-section slices of the tumor and colorectal wall providing a large surface area of tumor and healthy tissue. This method was chosen to obtain a sufficient amount of data to create a reliable classification. In a surgical setting these large surfaces of tumor will not be seen. Rectal tumors start developing in the mucosa and grow through the muscle layer into the surrounding mesorectal fat when becoming more advanced. In contrast to the large volume of tumor present in the lumen and wall of the rectum, smaller volumes of the tumor will be present in the mesorectal fat and possibly in the resection surface created by the surgeon. So the main question for future research should be whether the current classification will still be able to detect an area of tumor tissue which is much smaller compared to the cross-section slices and mainly surrounded by healthy tissue. So, as a next step towards in vivo use the entire resected specimens should be imaged with hyperspectral imaging to validate the current accuracy in a more realistic setting.

To be able to perform hyperspectral imaging during surgery some technical changes need to be made. The currently used set-up is a push-broom camera, where the samples are scanned by moving through the imaging line of the camera. In an in vivo setting, especially during laparoscopic surgery this will not be possible. Therefore, a snapshot multi-spectral camera should be used which can be attached to a laparoscopic system. In a multi-spectral camera a limited amount of wavelengths can be measured. These wavelengths should be chosen based on previous research. Therefore, further research should be performed on the selection of the most important wavelengths to distinguish tumor from healthy surrounding tissue. Using a snapshot camera will reduce the data acquisition time compared to the current set-up, from 1 minute to 1 second. Moreover, the preprocessing time of the data will decrease because of the limited number of wavelengths acquired. Therefore, when the current set-up is transformed into a set-up that can be used in vivo, real-time tissue classification will be possi- 
ble. Furthermore, in the in vivo setting, the measurements will be less controlled compared to the current study. For example the illumination of the tissue will be variable during surgery. Moreover, specular reflection and glare will be present. These issues should be taken into consideration before starting an in vivo study. Finally, when performing measurements in vivo a real-time classification should be available. The current classification method would allow such real-time use.

\subsection{Conclusion}

In this ex vivo study, fat, healthy colorectal wall and tumor tissue could be distinguished using $\mathrm{HSI}$ with an accuracy of $0.88(\mathrm{STD}=0.13)$. When the accuracy is determined per patient a mean accuracy of 0.93 (STD $=0.12$ ) was obtained. Two hyperspectral cameras were used, one in the visual wavelength range and one in the near-infrared wavelength range. Using only one of the two cameras decreased the accuracy for the visual and near-infrared camera. The results of this study show the potential of using HSI during colorectal surgery to increase the number of radical resections. Future research should be focused on imaging of entire specimen and the translation of the technique to an intra-operative setting. This should result in a technique that provides accurate real-time tissue classification during laparoscopic colorectal cancer surgery. 


\section{References}

[1] J. Ferlay et al., "Cancer incidence and mortality worldwide: Sources, methods and major patterns in GLOBOCAN 2012," International Journal of Cancer, vol. 136, no. 5, pp. E359-E386, 2015.

[2] L. Rabeneck, S. Horton, A. G. Zauber, and C. Earle, "Cancer: Disease Control Priorities," in Disease Control Priorities 3rd Edition, ch. 6, pp. 101-119, 2015.

[3] M. Pascual, S. Salvans, and M. Pera, "Laparoscopic colorectal surgery: Current status and implementation of the latest technological innovations," World Journal of Gastroenterology, vol. 22, pp. 704-717, jan 2016.

[4] M. V. Ottermo et al., "The Role of Tactile Feedback in Laparoscopic Surgery," Surgical Laparoscopy Endoscopy \& Percutaneous Techniques, vol. 16, no. 6, 2006.

[5] S. Schostek, M. O. Schurr, and G. F. Buess, "Review on aspects of artificial tactile feedback in laparoscopic surgery," Medical Engineering \& Physics, vol. 31, pp. 887-898, oct 2009.

[6] G. Lu and B. Fei, "Medical hyperspectral imaging: a review," Journal of Biomedical Optics, vol. 19, p. 10901, jan 2014.

[7] J. M. Benavides et al., "Multispectral digital colposcopy for in vivo detection of cervical cancer," Optics Express, vol. 11, no. 10, pp. 1223-1236, 2003.

[8] L. E. Boucheron et al., "Utility of multispectral imaging for nuclear classification of routine clinical histopathology imagery," BMC Cell Biology, vol. 8, pp. S8-S8, jul 2007.

[9] D. Hattery, M. Hassan, S. Demos, and A. Gandjbakhche, "Hyperspectral imaging of Kaposi's Sarcoma for disease assessment and treatment monitoring," in Applied Imagery Pattern Recognition Workshop, 2002. Proceedings., pp. 124-130, 2002.

[10] Z. Liu, H. Wang, and Q. Li, "Tongue Tumor Detection in Medical Hyperspectral Images," Sensors (Basel, Switzerland), vol. 12, pp. 162-174, dec 2012.

[11] B. Fei et al., "Label-free reflectance hyperspectral imaging for tumor margin assessment: a pilot study on surgical specimens of cancer patients," Journal of Biomedical Optics, vol. 22, p. 1, aug 2017.

[12] G. Lu et al., "Detection of Head and Neck Cancer in Surgical Specimens Using Quantitative Hyperspectral Imaging," Clinical Cancer Research, vol. 23, pp. 5426 LP - 5436, sep 2017.

[13] H. Akbari et al., "Cancer detection using infrared hyperspectral imaging," Cancer Science, vol. 102, pp. 852-857, apr 2011.

[14] S. Kiyotoki et al., "New method for detection of gastric cancer by hyperspectral imaging: a pilot study," vol. 18, pp. 26010-26017, 2013.

[15] K. Masood and N. M. N. M. Rajpoot, "Classification of colon biopsy samples by spatial analysis of a single spectral band from its hyperspectral cube," 2007. 
[16] K. Masood and N. M. N. M. Rajpoot, "Spatial analysis for colon biopsy classification from hyperspectral imagery," 2008.

[17] K. Rajpoot and N. Rajpoot, "SVM Optimization for Hyperspectral Colon Tissue Cell Classification," in Medical Image Computing and Computer-Assisted Intervention - MICCAI 2004 (C. Barillot, D. R. Haynor, and P. Hellier, eds.), (Berlin, Heidelberg), pp. 829-837, Springer Berlin Heidelberg, 2004.

[18] S. Rathore, M. Hussain, A. Ali, and A. Khan, "A Recent Survey on Colon Cancer Detection Techniques," IEEE/ACM Transactions on Computational Biology and Bioinformatics, vol. 10, no. 3 , pp. 545-563, 2013.

[19] M. Maggioni et al., "Hyperspectral microscopic analysis of normal, benign and carcinoma microarray tissue sections," vol. 6091, p. 60910I, International Society for Optics and Photonics, feb 2006.

[20] E. Claridge and D. Hidović-Rowe, "Model Based Inversion for Deriving Maps of Histological Parameters Characteristic of Cancer From Ex-Vivo Multispectral Images of the Colon," IEEE Transactions on Medical Imaging, vol. 33, no. 4, pp. 822-835, 2014.

[21] Z. Han et al., "In vivo use of hyperspectral imaging to develop a noncontact endoscopic diagnosis support system for malignant colorectal tumors," vol. 21, pp. 16001-16008, 2016.

[22] R. Kumashiro et al., "Integrated Endoscopic System Based on Optical Imaging and Hyperspectral Data Analysis for Colorectal Cancer Detection," Anticancer Research, vol. 36, pp. 3925-3932, aug 2016.

[23] Ocean Optics, "OOINLCorrect Loading Non-Linearity Correction Coefficients Instructions," tech. rep., Ocean Optics, Dunedin, 2012.

[24] J. Burger and P. Geladi, "Hyperspectral NIR image regression part I: calibration and correction," Journal of Chemometrics, vol. 19, pp. 355-363, may 2005.

[25] A.. Rinnan, F. van den Berg, and S. B. Engelsen, "Review of the most common pre-processing techniques for near-infrared spectra," TrAC Trends in Analytical Chemistry, vol. 28, pp. 12011222, nov 2009.

[26] E. J. M. Baltussen et al., "Diffuse reflectance spectroscopy as a tool for real-time tissue assessment during colorectal cancer surgery," Journal of biomedical optics, vol. 22, no. 10, pp. 106014106016, 2017.

[27] S. Boughorbel, F. Jarray, and M. El-Anbari, "Optimal classifier for imbalanced data using Matthews Correlation Coefficient metric," PloS one, vol. 12, no. 6, p. e0177678, 2017.

[28] T. M. Bydlon et al., "Chromophore based analyses of steady-state diffuse reflectance spectroscopy: current status and perspectives for clinical adoption," Journal of Biophotonics, vol. 8, pp. 9-24, jan 2015.

[29] J. W. Spliethoff et al., "Real-time in vivo tissue characterization with diffuse reflectance spectroscopy during transthoracic lung biopsy: a clinical feasibility study," Clinical Cancer Research, vol. 22, no. 2, pp. 357-365, 2015. 


\section{Chapter 4}

\section{Using diffuse reflectance spectroscopy to distinguish tumor tissue from fibrosis in rectal cancer patients as a guide to surgery}

Elisabeth J.M. Baltussen, Susan G. Brouwer de Koning, Joyce Sanders, Arend G.J. Aalbers, Niels F.M. Kok, Geerard L. Beets, Benno H.W. Hendriks, Henricus J.C.M. Sterenborg, Koert F.D. Kuhlmann, Theo J.M. Ruers, Lasers in Surgery and Medicine, 2019 


\section{Abstract}

In patients with rectal cancer who received neoadjuvant (chemo)radiotherapy, fibrosis is induced in and around the tumor area. Because tumor and fibrosis have similar visual and tactile feedback, they are hard to distinguish during surgery. To prevent positive resection margins during surgery and spare healthy tissue it would be of great benefit to have a real-time tissue classification technology that can be used in vivo. In this study, diffuse reflectance spectroscopy (DRS) was used to evaluate for real-time tissue classification of tumor and fibrosis. DRS spectra of fibrosis and tumor were obtained on excised rectal specimens. After normalization using the area under the curve, a support vector machine was trained using a ten-fold cross validation. Using spectra of pure tumor tissue and pure fibrosis tissue, we obtained a mean accuracy of 0.88 . This decreased to a mean accuracy of 0.61 when tumor measurements were used in which a layer of healthy tissue, mainly fibrosis, was present between the tumor and the measurement surface. It is possible to distinguish pure fibrosis from pure tumor. However, when the measurements on tumor also involve fibrotic tissue, classification accuracy decreases. 


\subsection{Introduction}

Colorectal cancer has an incidence of 19.7 per 100,000 in 2018, with about one third of the cases located in the rectum [1-3]. Surgery in combination with (chemo)radiotherapy is the standard of care for patients with locally advanced rectal cancer. The addition of (chemo)radiotherapy results in tumor shrinkage and fibrosis, which offers a better chance of radical resection and a decrease in local recurrence rate [4, 6-11]. However, fibrosis and tumor tissue have similar tactile feedback, which makes it hard for the surgeon to distinguish both. The ability to peroperatively discriminate fibrotic from tumorous tissue, would be of great benefit for the surgeon and could be of help in finding the delicate balance between complete removal of the tumor and preventing complications due to too extensive surgery. In rectal cancer complete removal of the tumor is defined by the circumferential resection margin (CRM), which is the shortest distance between the tumor and the resection surface. The CRM should be at least $1 \mathrm{~mm}$ to prevent local recurrence $[4,12]$.

Diffuse reflectance spectroscopy (DRS) has been used before as a technique to characterize tissue types, real-time [13]. In DRS, light from a broad band light source is sent to the tissue. Within the tissue, the light undergoes several interactions, like scattering and absorption. After a few interactions, part of the light is reflected to the surface of the tissue. The reflected light is collected and contains information about the specific absorption and scattering properties of the illuminated tissue type. The main absorbers in colorectal tissue are blood, water and fat. Using these reflectance spectra, different tissue types can often be distinguished [14].

In various cancer types like breast, liver, lung, head and neck and colorectal cancer, DRS has been used to distinguish tumor from healthy surrounding tissue [1522]. In colorectal cancer the focus has mainly been on tissue discrimination during colonoscopy [13, 23-25]. However, in surgery the rectum is approached from outside the lumen, whereas in colonoscopy the approach is from inside the lumen. A study by Schols et al. did perform DRS measurements on colorectal tissue in a surgical setting. They found that it was possible to distinguish tumor tissue from surrounding colorectal tissues with an accuracy of 0.99 (STD=0.19) [19]. In two previous studies, performed by our group, similar results were found in the discrimination of tumor tissue from healthy surrounding tissue in the colon [15, 20]. Langhout et al. [15] obtained an accuracy of $91 \%$ and Baltussen et al. an accu- 
racy of 0.95 (STD=0.03) [20]. However, none of these studies included fibrosis in the healthy tissue types, while neoadjuvant (chemo)radiotherapy, which is standard care for patients with locally advanced rectal cancer, induces fibrosis in and around the tumor area $[15,19,20]$.

To investigate whether DRS could be of use during surgery of advanced rectal cancer, we evaluated if the technique was able to differentiate tumor tissue form fibrosis in resection specimens of advanced rectal cancer patients who were pretreated with chemoradiation. The ultimate goal would be to incorporate DRS into smart surgical tools ready for real time surgical guidance.

\subsection{Materials and methods}

\subsubsection{DRS system}

The system consists of two spectrometers, one resolves light from 400 to $1100 \mathrm{~nm}$ (Andor Technology, DU420ABRDD), the other from 900 to $1700 \mathrm{~nm}$ (Andor Technology, DU492A-1.7). Furthermore, the system has a Tungsten halogen broadband light source with an embedded shutter. The light source covers the visual and infrared wavelength range. The system was controlled using custom-made LabView software (National Instruments). The measurements were performed using a fiber optics probe that consisted of three fibers with a core diameter of $200 \mu \mathrm{m}$ (Fig. 4.1). One fiber was used to transport light from the source to the tissue, the other two were connected to the spectrometers to transport the reflected light from the tissue surface to the spectrometers. All specimens were measured using the same probe. The calibration of the system including the probe was previously described by Nachabé et al. [26, 27]. In short a white reference, a measurement on Spectralon, was performed before the start of the measurements on each patient. Furthermore, a dark reference was measured by closing the shutter before each single measurement. Using the white and dark reference all measurements were calibrated using Equation 4.1. In Equation 4.1 the $R_{c a l}$ is the calibrated measurement, $R_{\text {meas }}$ is the uncalibrated measurement and $R_{\text {white }}$ and $R_{\text {dark }}$ are the white and dark reference measurements respectively.

$$
R_{\text {cal }}=\frac{R_{\text {meas }}-R_{\text {dark }}}{R_{\text {white }}-R_{\text {dark }}}
$$

The sampling depth of the DRS probe is dependent upon the optical properties 


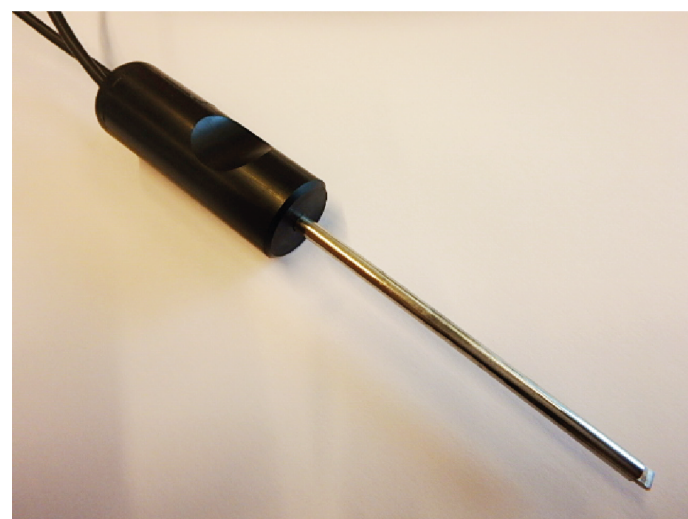

\section{Contacting surface of the probe}

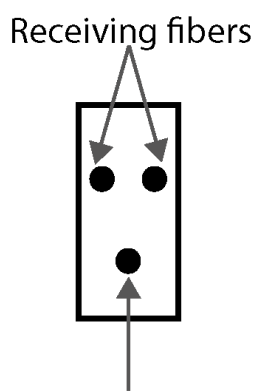

Emitting fiber

Figure 4.1: Image of the probe used in this study.

of the tissue, the wavelength of the light and the distance between the delivering and receiving fibers and as a rule of thumb is similar to the distance between the emitting and receiving fiber [28]. In this study a distance of $2 \mathrm{~mm}$ between the delivering and receiving fibers was used. This will result in a sampling depth of about $2-3 \mathrm{~mm}$.

\subsubsection{Study protocol}

The study was performed in the Netherlands Cancer Institute under approval of the protocol by the Hospital Ethics Review Board. Thirty-eight patients undergoing surgery for a locally advanced rectal tumor were included in the study. All ethical guidelines were followed.

Immediately after surgery, ultrasound images of the specimen were obtained to localize the area with the smallest distance from the surface of the specimen to tumor or fibrosis. Based on the ultrasound images, three DRS point measurements were performed on or close to the tumor or fibrotic area. Measurements were performed within one hour after resection and locations were marked using ink. Acquisition time of one measurement was a few seconds. After the measurements, the specimen was brought to the Pathology Department, were it was processed according to standard protocol. Pathology slides of all measurement locations were obtained. These pathology slides were annotated by a pathologist, who marked the various tissue types present in the slides. Based on these annotations the measured spectra were classified. A measurement was marked as pure if only one tissue type was present, within the probed volume according 
to the histopathological examination.

Because only a small number of pure tumor measurements were obtained on the included specimens, making classification less reliable, tumor measurements of another study performed on cross-sections of colon tumors were used in a first step to obtain a classification on tumor and fibrosis. The cross-sections were taken from the freshly excised specimen, before fixation and without freezing the specimen, and were about $5 \mathrm{~mm}$ thick. The measurements on these crosssections were obtained using the same measurement set-up and probe [20].

\subsubsection{Data analysis}

Data analysis was performed using Matlab (version 8.5, MatWorks Inc., Natick, Massachusetts). Calibration of the spectra was performed using a white and dark reference taken before the measurements of each patient. After data acquisition, the spectra from the two spectrometers were stitched together, by multiplying the entire spectrum of one spectrometer with a factor to align the intensity of the wavelength range from $900-1100 \mathrm{~nm}$, which is acquired with both spectrometers, to the intensity of the spectrum obtained with the other spectrometer. Thereafter, all spectra were normalized by setting the area under the curve (AUC) of all spectra to one. Spectral analysis was done on the normalized spectra to visualize the potential of discriminating tumor tissue from fibrosis. Measurements with a layer of fibrosis between tumor and the measurement surface, of maximum $2 \mathrm{~mm}$, were also included in the analysis. The normalized spectra were used to train a linear support vector machine (SVM) using a tenfold cross-validation to distinguish tumor from fibrosis. A linear SVM was chosen as classification method because the method performed well in earlier studies, also when compared to other classification methods $[20,5]$. The train and test datasets used for this first SVM consisted of fibrosis data obtained in the current study and pure tumor data obtained in the previous study (Fig. 4.2).

A second linear SVM was trained and tested, again using a tenfold cross-validation which was repeated ten times, on data that was obtained only in the current study (Fig. 4.2). For this classification, measurements with tumor within $2 \mathrm{~mm}$ in depth from the measured surface were classified as tumor. The limit of $2 \mathrm{~mm}$ was chosen because the measurement depth was about $2 \mathrm{~mm}$ with a fiber distance of 2 $\mathrm{mm}$. Evaluation of both classifications was done using the accuracy, sensitivity, specificity and the Matthews Correlation Coefficient (MCC). The MCC is added as 

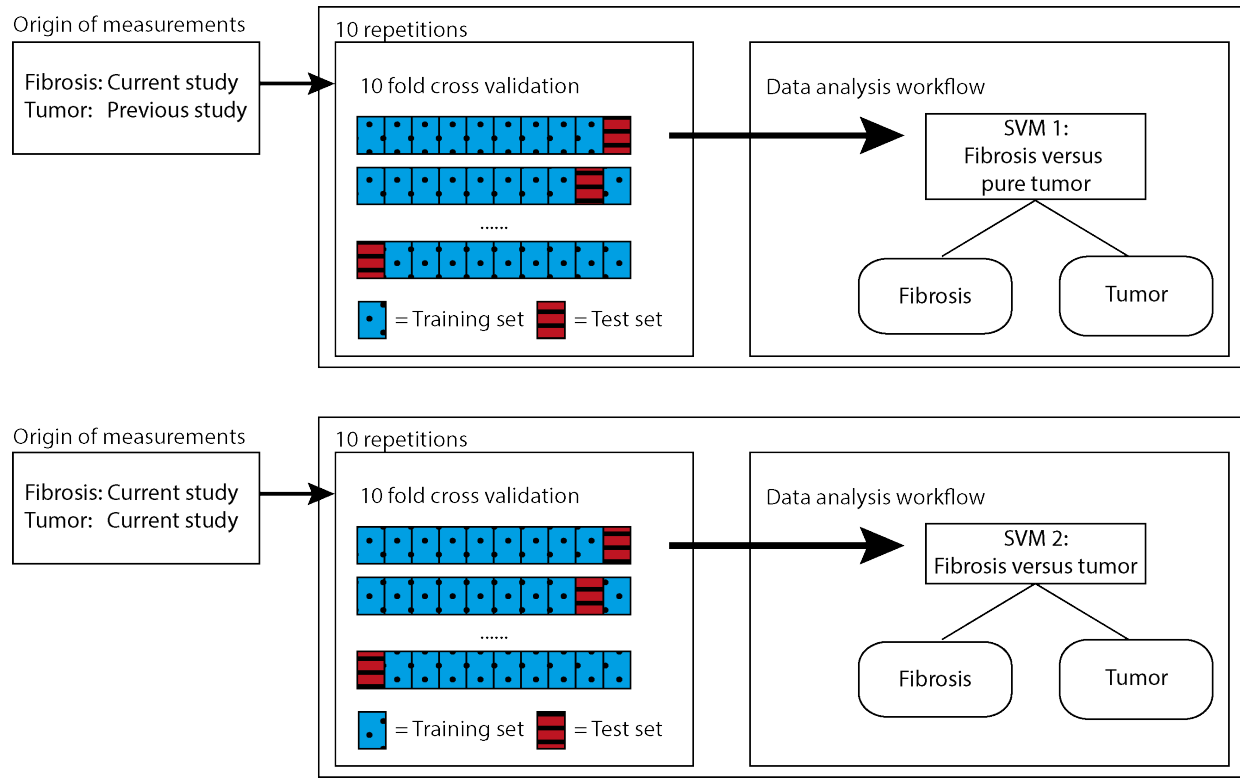

Figure 4.2: Data analysis structure

performance measure because it is less influenced by the large number of fibrosis measurements compared to the number of tumor measurements in the dataset. The MCC is calculated according to Equation 2 and returns a value between 1 and 1 , where 0 indicates a performance no better than random, -1 indicates complete disagreement and +1 complete agreement. In Equation 4.2 TN, TP, FN and FP are the number of true negatives, true positives, false negatives and false positives respectively.

$$
M C C=\frac{T P \times T N-F P \times F N}{\sqrt{(T P+F P)(T P+F N)(T N+F P)(T N+F N)}}
$$

\subsection{Results}

\subsubsection{Inclusion}

In total 38 patients with rectal cancer were included in this study. In Table 4.1 patient and tumor characteristics are summarized. Most patients received capecitabine, sometimes combined with oxaliplatin and bevacizumab as chemotherapy. Radiotherapy mostly consisted of 25 fractions of 2 gray ( 15 patients), followed by 
5 fractions of 5 gray (11 patients). The other radiotherapy courses included unknown number of courses ( 6 patients), 30 gray in 5 fractions ( 1 patient), 90 gray in 3 fractions ( 1 patient) and a combination of 50 gray and 90 gray (1 patient). The median time between the last course of radiotherapy and surgery was 4.8 months (interquartile range 3.3-7.7). Three patients without neoadjuvant radiotherapy were included to increase the chances of measuring tumor at the surface.

Table 4.1: Patient and tumor characteristics

\begin{tabular}{llc}
\hline Number of patients & & 38 \\
\hline \multirow{2}{*}{ Gender } & Male & 27 \\
& Female & 11 \\
\hline \multirow{2}{*}{ Age } & Median (years) & 63.5 \\
& Interquartile range & $57-68$ \\
\hline \multirow{3}{*}{ Tumor stage } & pT0 & 2 \\
& pT1 & 0 \\
& pT2 & 3 \\
& pT3 & 29 \\
& pT4 & 4 \\
\hline \multirow{2}{*}{ Neoadjuvant therapy } & Chemotherapy & 1 \\
& Radiotherapy & 4 \\
& Chemoradiotherapy & 31 \\
\multirow{2}{*}{ Time end radiotherapy - surgery } & None & 2 \\
\hline \multirow{2}{*}{ CRM } & Median (months) & 4.8 \\
& Interquartile range & $3.3-7.7$ \\
\hline
\end{tabular}

The pathology analysis found positive CRMs in 6 out of 38 patients, defined as tumor within $1 \mathrm{~mm}$ of the resected surface. In 2 of these cases, tumor tissue was present within the first $1 \mathrm{~mm}$ below the resection margin, but not at the surface. In four out of 6 patients with a positive CRM, tumor was found directly at the surface of the resected specimen. The area of tumor at the surface of the specimen was in all patients less than $1 \mathrm{~cm}$. In one additional patient, tumor was found at the surface of the specimen. However, this was not at the resection surface because it was located in the sigmoid part of the resection specimen. Therefore, no positive CRM was noted for this patient.

To obtain a ground truth for all measurements histopathology was analyzed for all measurements. In this analysis the thickness of the top layer was taken into account for the tumor measurements. If tumor was more than $3 \mathrm{~mm}$ below the surface, e.g. a top layer thickness of $>3 \mathrm{~mm}$, the measurement at this location 
was not included in the tumor measurements. When analyzing the histopathology of the locations measured by DRS (three locations per patient, which were all included in the classification), tumor was found at the surface of 5 measured locations (4 patients). In one of these locations the tumor had a thickness of only $1.3 \mathrm{~mm}$, in all other cases the tumor was at least $5 \mathrm{~mm}$ thick. In 12 locations (8 patients) tumor was measured within $2 \mathrm{~mm}$ from the surface (Fig. 4.3a). In 16 locations (11 patients), the distance between tumor and the surface was between 2 and $3 \mathrm{~mm}$. All other locations ( 30 patients) had a distance of at least $3 \mathrm{~mm}$ between the measurement surface and tumor (Fig. 4.3b). In Table 4.2 an overview is given for all measurements, together with the thickness of the given tissue type between the measurement surface and tumor. For the locations noted in the first three to four rows of this table it would have been possible to measure tumor tissue given the fiber distance of $2 \mathrm{~mm}$, and an approximate measurement depth of $2-3 \mathrm{~mm}$.

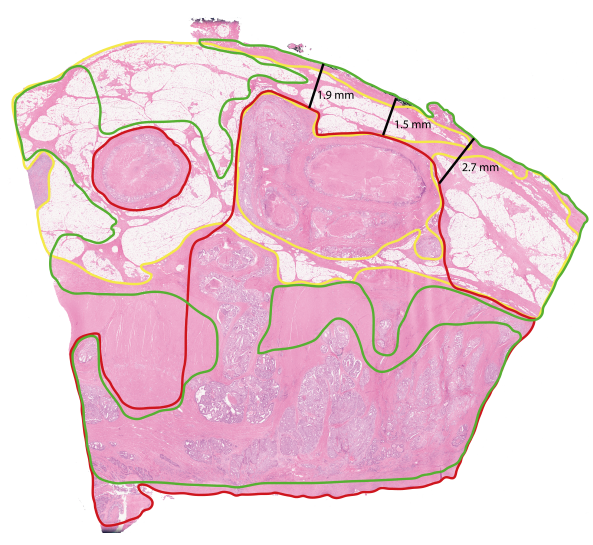

(a) Locations that do include tumor in measured volume.

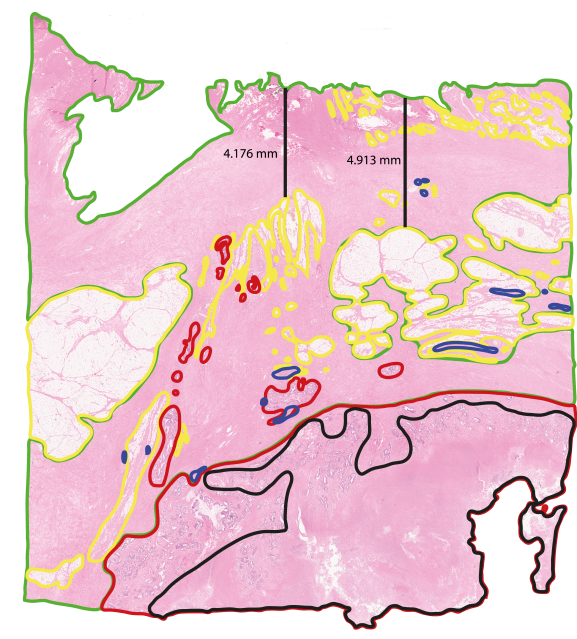

(b) Locations that do not include tumor in measured volume

Figure 4.3: Pathology slides annotated by the pathologist, red $=$ tumor, yellow $=$ fat, green $=$ fibrosis, blue $=$ inflammation, black $=$ necrosis.

\subsubsection{Spectral analysis}

In total 117 measurements were performed, because in one patient 6 measurements instead of 3 were done. In Figure $4.4 a$, the mean spectra are shown of pure fibrosis measurements, pure tumor measurements and measurements 
Table 4.2: Number of locations per distance from measurement surface to tumor and per tissue type

\begin{tabular}{lcccc}
\hline & \multicolumn{4}{c}{ Number of measurement locations } \\
Thickness of top layer & Fibrosis & Healthy rectal wall & Fat & Total \\
\hline $0 \mathrm{~mm}$ & - & - & - & 5 \\
$>0-1 \mathrm{~mm}$ & 4 & 0 & 0 & 4 \\
$>1-2 \mathrm{~mm}$ & 6 & 1 & 1 & 8 \\
$>2-3 \mathrm{~mm}$ & 9 & 5 & 2 & 16 \\
$>3-4 \mathrm{~mm}$ & 4 & 1 & 3 & 8 \\
$>4-5 \mathrm{~mm}$ & 7 & 2 & 6 & 15 \\
$>5-6 \mathrm{~mm}$ & 1 & 2 & 4 & 7 \\
$>6-7 \mathrm{~mm}$ & 0 & 1 & 4 & 5 \\
$>7-8 \mathrm{~mm}$ & 2 & 2 & 0 & 4 \\
$>8-9 \mathrm{~mm}$ & 3 & 1 & 1 & 5 \\
$>9-10 \mathrm{~mm}$ & 3 & 0 & 0 & 3 \\
$\leq 10 \mathrm{~mm}$ & 12 & 9 & 16 & 37 \\
\hline
\end{tabular}

with fibrosis between the measurement surface and the tumor, all measurements were performed in the current study. Different thicknesses of the fibrotic layer between tumor and the measurement surface are shown separate in Figure $4.4 \mathrm{~b}$ 4.4h. There, the mean spectra are shown separately with one standard deviation. From Figure $4.4 \mathrm{a}$, the pure tumor measurements can be separated quite well from all others, whereas the distinction of pure fibrosis measurements from measurements of tumor with a layer of fibrosis between tumor and the measurement surface is more difficult.

\subsubsection{Classification results}

A linear SVM was trained and tested on a combination of spectra from pure fibrosis measurements from the current study and pure tumor measurements from the previous study. This was done, since the small number of pure tumor samples in the current study made tissue classification less reliable. Since 97 locations with pure fibrosis were measured, in 11 patients, and included in the analysis, 96 locations of pure tumor measurements from 11 patients were randomly selected from the previous study for classification. Using this data in a tenfold cross-validation training and testing of the SVM, which was repeated ten times, resulted in a mean accuracy of $0.88(S T D=0.02)$, a mean sensitivity and specificity of 0.91 (STD = 0.01 ) and $0.86(S T D=0.03)$ respectively and a mean MCC of $0.76(S T D=0.03)$. A second linear SVM was trained and tested using only data from the current study. This resulted in a dataset in which almost no pure tumor measurements were present but most were mixed with other tissue types. Measurement locations 


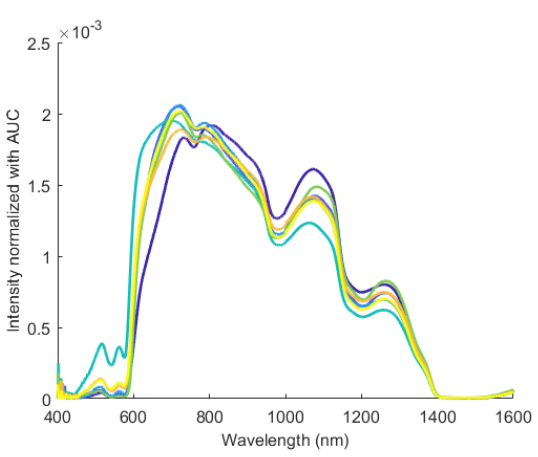

(a)

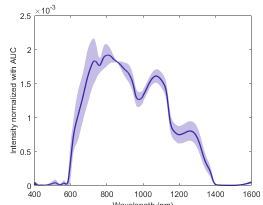

(b)

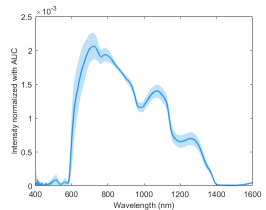

(d)

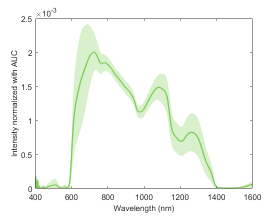

(f)

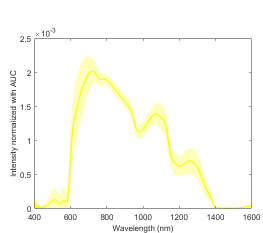

(h)

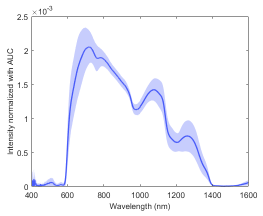

(c)

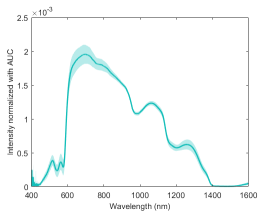

(e)

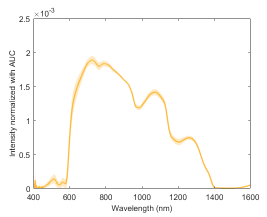

(g)

Pure tumor

0-1 mm fibrosis

1-2 $\mathrm{mm}$ fibrosis

2-3 $\mathrm{mm}$ fibrosis

3-4 mm fibrosis

4-5 mm fibrosis - Pure fibrosis

Figure 4.4: (a) Mean spectra of pure tumor and pure fibrosis measurements and of measurements with a layer of fibrosis between the measurement location and tumor. (b)-(h) Mean spectra ( \pm 1 STD) of pure tumor, $>0-1 \mathrm{~mm}$ fibrosis above tumor, $>1-2 \mathrm{~mm}$ fibrosis above tumor, $>2-3 \mathrm{~mm}$ fibrosis above tumor, $>3-4 \mathrm{~mm}$ fibrosis above tumor, $>4-5 \mathrm{~mm}$ fibrosis above tumor and pure fibrosis respectively. 
were classified as tumor, if tumor was present within $2 \mathrm{~mm}$ from the measurement surface. With this classifier a mean accuracy of 0.61 (STD $=0.05$ ), a mean sensitivity and specificity of $0.51(S T D=0.10)$ and $0.66(S T D=0.11)$ respectively and a mean MCC of 0.17 (STD $=0.08$ ) were obtained. In Figure 4.5 the mean ROC-curves of the ten repetitions of both SVM's are shown, visualizing the clear drop in performance between the first and second SVM.

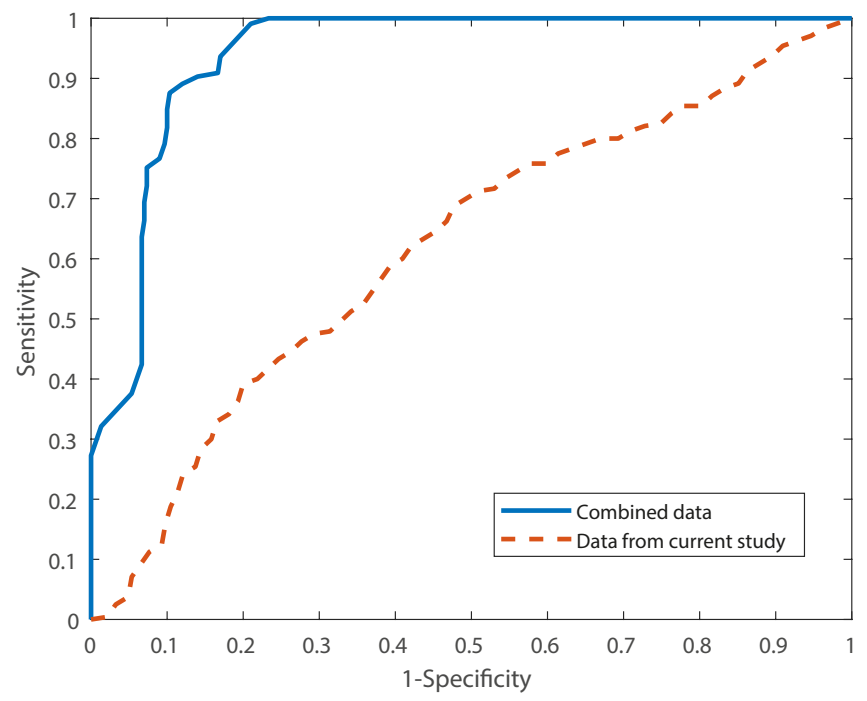

Figure 4.5: Mean ROC-curves of the ten repetitions of the 10-fold cross validation of the two linear SVMs

\subsection{Discussion}

In the current study we tried to create a classifier to distinguish tumor from fibrosis in resection specimen of patients with advanced rectal cancer who received (chemo)radiotherapy. If pure tumor and pure fibrosis measurements are used for the classification a mean accuracy of 0.88 and a mean MCC of 0.76 is obtained. However, both values decrease when pure tumor measurements are replaced by a measurements on a combination of tumor tissue with healthy tissue.

After surgery, in 6 out of 38 patients a positive CRM was found by the pathologist, which is about $16 \%$. We were only able to obtain 5 measurements, in 4 patients, directly onto tumor tissue. Because this number was too low to obtain 
a reliable classification of tumor and fibrosis, it was decided to add pure tumor measurements from a previous study to the dataset. These measurements were obtained using the same measurement set-up and the same probe. However, measurements in the previous study were performed on cross-sections of colon tumors [20]. Classification of the pure tumor and pure fibrosis measurements could be done with an accuracy of 0.88 and an MCC of 0.76 . These results are similar to the results obtained to distinguish tumor from healthy colorectal wall in earlier studies [15, 19, 20]. However, in these studies fibrosis was not included as a healthy tissue type. Distinguishing tumor from fat or tumor from healthy colorectal wall is most likely easier compared to distinguishing tumor from fibrosis. This is mainly due to the fact that fibrosis appears in and around the tumor and spectra from these regions might thus be similar.

If however, only measurements from the current study are taken into account, the accuracy and MCC drop to 0.61 and 0.17 respectively. There are several possible explanations for the lower accuracy and MCC value. First of all, the SVM trained on only pure tumor and fibrosis measurements might not be classifying based upon tissue specific differences between tumor and fibrosis. Instead, it might be that the classification is done based upon differences between the two different datasets, even though the measurement set-up and probe used in both studies were the same. This difference could have originated due to the difference in tissue samples. In the current study entire rectal specimens were measured from the surface whereas in the previous study tissue samples were measured. A second explanation for the lower accuracy and MCC value could be that in the train data of the second SVM only 4 pure tumor measurements were present. This makes it harder to train a correct classification. The increase in standard deviation for the second classification supports this explanation. The extent of the influence of both explanations is hard to determine.

In Figure 4.4, the mean spectra of pure fibrosis, pure tumor and measurements with a layer of fibrosis on top of tumor are shown, all spectra were obtained in the current study. The mean tumor spectrum can best be distinguished from the rest. However, it is hard to distinguish the measurements with the different layer thicknesses of fibrosis on top of tumor from each other and from the pure fibrosis measurements. This shows the difficulty of the classification problem. Although it is possible to distinguish pure tumor measurements from pure fibrosis measurements, as shown by the results of the first SVM as well as the spectral analysis in Figure $4.4 \mathrm{a}$, it is hard to distinguish fibrosis from a mixture of tumor and fibrosis, 
as shown by the results of the second SVM. In the pathology reports from the patients included in this study, a positive CRM of less than $1 \mathrm{~cm}$ in length, sometimes even discontinuous, is most often described by the pathologist. While we show that pure tumor tissue can be differentiated form pure fibrosis it remains to be determined whether it is possible to detect relatively small tumor areas, especially when they are covered or surrounded by fibrosis. Based upon Figure 4.4 this might be challenging.

To be able to give a more funded answer to this question, more measurements should be obtained with different amounts of tumor and fibrosis. Given the low numbers of pure tumor measurements in the current series, and probably also in future series, this will require a considerable effort. With the increasing use of neoadjuvant (chemo)radiotherapy, the need to distinguish tumor from fibrosis and other healthy tissues during surgery remains important. Intensification of neoadjuvant treatment allows that more healthy tissue can be spared during surgery, decreasing the number of complications. To be able to spare extra healthy tissue, a technique able to discriminate fibrosis from tumor tissue is still highly needed. Diffuse reflectance spectroscopy could be a potential candidate, but further evaluation of the technology in larger series is necessary.

\subsection{Conclusion}

In this ex vivo study, we found that it is possible to distinguish pure tumor from pure fibrosis measurements with a mean accuracy of $0.88(\mathrm{MCC}=0.76)$. If the classification is based on measurements on a mixture of healthy tissue with tumor, the mean classification accuracy drops to $0.61(\mathrm{MCC}=0.17)$. The main challenge for DRS will be to detect tumor, in a mixture of tumor and fibrosis 


\section{References}

[1] J. Ferlay, M. Ervik, and F. Lam, "Global Cancer Observatory: Cancer Today." https://gco.iarc.fr/today/data/factsheets/cancers/10_8_9-Colorectum-fact-sheet.pdf, 2018.

[2] F. Bray et al., "Global cancer statistics 2018: GLOBOCAN estimates of incidence and mortality worldwide for 36 cancers in 185 countries," CA: A Cancer Journal for Clinicians, vol. 68, pp. 394424, nov 2018.

[3] R. Glynne-Jones et al., "Rectal cancer: ESMO Clinical Practice Guidelines for diagnosis, treatment and follow-upt," Annals of Oncology, vol. 28, pp. iv22-iv40, jul 2017.

[4] I. D. Nagtegaal and P. Quirke, "What Is the Role for the Circumferential Margin in the Modern Treatment of Rectal Cancer?," Journal of Clinical Oncology, vol. 26, no. 2, pp. 303-312, 2008.

[5] E. J. M. Baltussen, H. J. C. M. Sterenborg, T. J. M. Ruers, B. Dashtbozorg "Optimizing algorithm development for tissue classification in colorectal cancer based on diffuse reflectance spectra," Biomedical Optics Express, accepted, 2019.

[6] J. Garcia-Aguilar et al., "Optimal Timing of Surgery After Chemoradiation for Advanced Rectal Cancer: Preliminary Results of a Multicenter, Nonrandomized Phase II Prospective Trial," Annals of Surgery, vol. 254, no. 1, 2011.

[7] S. T. Martin, H. M. Heneghan, and D. C. Winter, "Systematic review and meta-analysis of outcomes following pathological complete response to neoadjuvant chemoradiotherapy for rectal cancer," BJS, vol. 99, pp. 918-928, feb 2012.

[8] R. Sauer et al., "Preoperative versus Postoperative Chemoradiotherapy for Rectal Cancer," New England Journal of Medicine, vol. 351, pp. 1731-1740, oct 2004.

[9] S. Pucciarelli et al., "Long-Term Oncologic Results and Complications After Preoperative Chemoradiotherapy for Rectal Cancer: A Single-Institution Experience After a Median FollowUp of 95 Months," Annals of Surgical Oncology, vol. 16, no. 4, p. 893, 2009.

[10] L. F. de Campos-Lobato et al., "Pathologic Complete Response After Neoadjuvant Treatment for Rectal Cancer Decreases Distant Recurrence and Could Eradicate Local Recurrence," Annals of Surgical Oncology, vol. 18, no. 6, pp. 1590-1598, 2011.

[11] K. D. Smith et al., "Clinical Significance of Acellular Mucin in Rectal Adenocarcinoma Patients With a Pathologic Complete Response to Preoperative Chemoradiation," Annals of Surgery, vol. 251, no. 2, 2010.

[12] C. Simillis et al., "A systematic review to assess resection margin status after abdominoperineal excision and pelvic exenteration for rectal cancer," Annals of surgery, vol. 265, no. 2, pp. 291299, 2017.

[13] G. Zonios et al., "Diffuse reflectance spectroscopy of human adenomatous colon polyps in vivo," Applied optics, vol. 38, no. 31, pp. 6628-6637, 1999. 
[14] D. J. Evers, B. H. W. Hendriks, G. W. Lucassen, and T. J. M. Ruers, "Optical spectroscopy: current advances and future applications in cancer diagnostics and therapy," Future Oncology, vol. 8, no. 3, pp. 307-320, 2012.

[15] G. C. Langhout et al., "Differentiation of healthy and malignant tissue in colon cancer patients using optical spectroscopy: A tool for image-guided surgery," Lasers in Surgery and Medicine, vol. 47, no. 7, pp. 559-565, 2015.

[16] D. J. Evers et al., "Optical sensing for tumor detection in the liver," European Journal of Surgical Oncology, vol. 39, no. 1, pp. 68-75, 2013.

[17] L. L. Boer et al., "Fat/water ratios measured with diffuse reflectance spectroscopy to detect breast tumor boundaries," Breast Cancer Research and Treatment, vol. 152, no. 3, pp. 509-518, 2015.

[18] J. W. Spliethoff et al., "Improved identification of peripheral lung tumors by using diffuse reflectance and fluorescence spectroscopy," Lung Cancer, vol. 80, no. 2, pp. 165-171, 2013.

[19] R. M. Schols, P. Dunias, F. P. Wieringa, and L. P. S. Stassen, "Multispectral characterization of tissues encountered during laparoscopic colorectal surgery," Medical Engineering and Physics, vol. 35, no. 7, pp. 1044-1050, 2013.

[20] E. J. M. Baltussen et al., "Diffuse reflectance spectroscopy as a tool for real-time tissue assessment during colorectal cancer surgery," Journal of biomedical optics, vol. 22, no. 10, pp. 106014106016, 2017.

[21] C.-C. Yu et al., "Quantitative spectroscopic imaging for noninvasive early cancer detection," Opt. Express, vol. 16, pp. 16227-16239, sep 2008.

[22] S. G. Brouwer de Koning et al., "Toward complete oral cavity cancer resection using a handheld diffuse reflectance spectroscopy probe," Journal of Biomedical Optics, vol. 23, pp. 1-9, oct 2018.

[23] H.-W. Wang et al., "Diffuse reflectance spectroscopy detects increased hemoglobin concentration and decreased oxygenation during colon carcinogenesis from normal to malignant tumors.," Optics express, vol. 17, no. 4, pp. 2805-17, 2009.

[24] X. Shao, W. Zheng, and Z. Huang, "In vivo diagnosis of colonic precancer and cancer using near-infrared autofluorescence spectroscopy and biochemical modeling.," Journal of biomedical optics, vol. 16, no. 6, p. 067005, 2011.

[25] H. K. Roy et al., "Spectroscopic Microvascular Blood Detection From the Endoscopically Normal Colonic Mucosa: Biomarker for Neoplasia Risk," Gastroenterology, vol. 135, no. 4, pp. 10691078, 2008.

[26] R. Nachabé et al., "Estimation of biological chromophores using diffuse optical spectroscopy: benefit of extending the UV-VIS wavelength range to include 1000 to $1600 \mathrm{~nm}$," Biomedical Optics Express, vol. 1, no. 5, pp. 1432-1442, 2010.

[27] R. Nachabe et al., "Estimation of lipid and water concentrations in scattering media with diffuse optical spectroscopy from 900 to 1600 nm," vol. 15, pp. 37010-37015, 2010.

[28] A. J. Gomes and V. Backman, "Algorithm for automated selection of application-specific fiberoptic reflectance probes," Journal of Biomedical Optics, vol. 18, p. 27012, feb 2013. 


\section{Chapter 5}

\section{Tissue diagnosis during colorectal cancer surgery using optical sensing: an in vivo study}

Elisabeth J.M. Baltussen, Susan G. Brouwer de Koning, Joyce Sanders, Arend G.J. Aalbers, Niels F.M. Kok, Geerard L. Beets, Benno H.W. Hendriks, Henricus J.C.M. Sterenborg, Koert F.D. Kuhlmann, Theo J.M. Ruers, Journal of Translational Medicine, 2019, Volume 17(1) 


\section{Abstract}

In colorectal cancer surgery there is a delicate balance between complete removal of the tumor and sparing as much healthy tissue as possible. Especially in rectal cancer, intraoperative tissue recognition could be of great benefit in preventing positive resection margins and sparing as much healthy tissue as possible. To better guide the surgeon, we evaluated the accuracy of diffuse reflectance spectroscopy (DRS) for tissue characterization during colorectal cancer surgery and determined the added value of DRS when compared to clinical judgement. DRS spectra were obtained from fat, healthy colorectal wall and tumor tissue during colorectal cancer surgery and results were compared to histopathology examination of the measurement locations. All spectra were first normalized at 800 $\mathrm{nm}$, thereafter two support vector machines (SVM) were trained using a tenfold cross-validation. With the first SVM fat was separated from healthy colorectal wall and tumor tissue, the second SVM distinguished healthy colorectal wall from tumor tissue. Patients were included based on preoperative imaging, indicating advanced local stage colorectal cancer. Based on the measurement results of 32 patients, the classification resulted in a mean accuracy for fat, healthy colorectal wall and tumor of $0.92,0.89$ and 0.95 respectively. If the classification threshold was adjusted such that no false negatives were allowed, the percentage of false positive measurement locations by DRS was $25 \%$ compared to $69 \%$ by clinical judgement. This study shows the potential of DRS for the use of tissue classification during colorectal cancer surgery. Especially the low false positive rate obtained for a false negative rate of zero shows the added value for the surgeons. This trail was performed under approval from the internal review board committee (Dutch Trail Register NTR5315). 


\subsection{Introduction}

Colorectal cancer is the third most common cancer worldwide [1]. Most tumors are located in the proximal colon (41\%) followed by the rectum (28\%) [2]. Surgery is the standard treatment, while patients with advanced rectal cancer are generally treated with a combination of neoadjuvant chemo- and radiotherapy [3]. In rectal cancer surgery there is a delicate balance between the complete removal of the tumor and sparing of vital surrounding tissue such as blood vessels, nerves and ureters. Damage to these structures leads to complications such as bladder and sexual dysfunction $[4,5]$. In addition, the surgeon is confronted with limited space in the pelvic cavity as well as with fibrotic tissue induced by (chemo)radiotherapy. This often further impedes the determination of the exact tumor borders. These circumstances might lead to a positive resection margin (CRM), which is generally defined as tumor tissue within $2 \mathrm{~mm}$ from the resection surface. A positive $\mathrm{CRM}$ is a negative independent predictor of survival and local recurrence $[6,7]$. Intraoperative tissue recognition could decrease the number of positive CRMs, while preventing complications that are caused by too extensive surgery. Currently there is no technique available which allows such intraoperative tissue type characterization in rectal cancer surgery.

Diffuse reflectance spectroscopy (DRS) might offer the possibility for intraoperative tissue recognition. In DRS, light from a broadband light source is sent into the tissue. In the tissue, the light undergoes several interactions such as scattering and absorption, before part of the light will be reflected back to the surface of the tissue. This light is collected and will form a spectrum which can be analyzed. The shape of the collected spectrum depends on the constituents of the tissue the light went through, which potentially allows discrimination of different tissue types [8]. DRS has already been successfully used in several different cancer types to distinguish between tumor and healthy tissue, e.g. in lung, breast, liver and head and neck cancer with accuracies of at least $77 \%$ [8-13].

So far, the use of DRS in colorectal cancer focused mainly on the application in colonoscopy [14-18]. These studies were performed in vivo and obtained sensitivities and specificities between $80-92 \%$ and $75-78 \%$, respectively, for the detection of cancer tissue versus healthy tissue $[16,17]$. The main difference between colonoscopy and a surgical setting is the fact that in colonoscopy tissue is assessed from inside the lumen, whereas in surgery the tissue is assessed from outside the lumen. Ex vivo studies focusing on DRS in colorectal surgery showed 


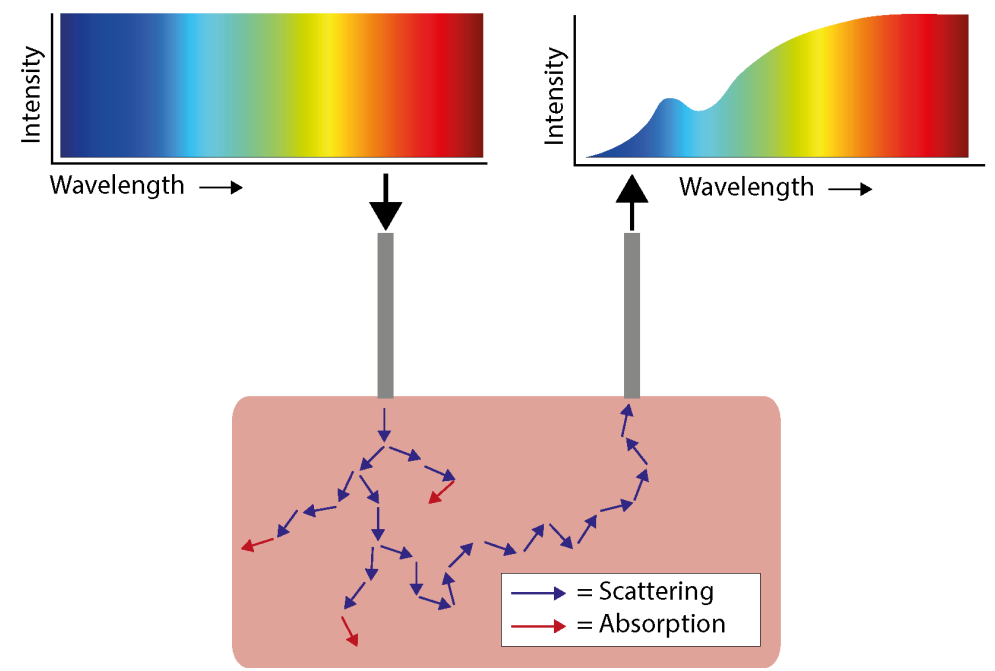

Figure 5.1: Basic principle of DRS. Light, with a broad wavelength range, is send through a fiber to the tissue. Within the tissue this light undergoes several interactions like scattering (blue arrows) and absorption (red arrows). Part of the light will be scattered to the surface where it is detected using a second fiber. The detected signal will be different than the signal that was send into the tissue due to the specific absorption of the tissue constituents. Based on the signal alterations different tissue types can be discriminated.

that tumor can be distinguished from healthy surrounding tissue with an accuracy of at least $91 \%$ [19-21].

This study investigates the role of DRS in colorectal cancer surgery in vivo. DRS measurement locations were determined by the surgeon and were located at the tumor and healthy surrounding tissues. The analysis of the measurements was done offline after surgery and was verified by pathological assessment. The aim of the study was to determine the accuracy of the DRS measurements in a surgical setting and to evaluate the added value when compared to the clinical judgement of the surgeon. Ultimately this could lead to a smart surgical tool for real-time peroperative tissue classification allowing more precise surgery.

\subsection{Materials and methods}

\subsubsection{DRS system}

The DRS system consists of two spectrometers, a Tungsten halogen broadband light source and an embedded shutter. The light source covers the visual and 
infrared wavelength range from 360 to $2500 \mathrm{~nm}$. The two spectrometers cover most of this wavelength range as well, with one covering the visual wavelengths, 400 to $1100 \mathrm{~nm}$, (Andor Technology, DU420ABRDD). The other spectrometer covers the near-infrared wavelength range of 900-1700 nm (Andor Technology, DU492A-1.7) (Figure 2). Custom made LabView software (National Instruments, Austin, Texas) makes it possible to control the system and to acquire and save the data. A detailed description about calibration of the system can be found elsewhere [22, 23].
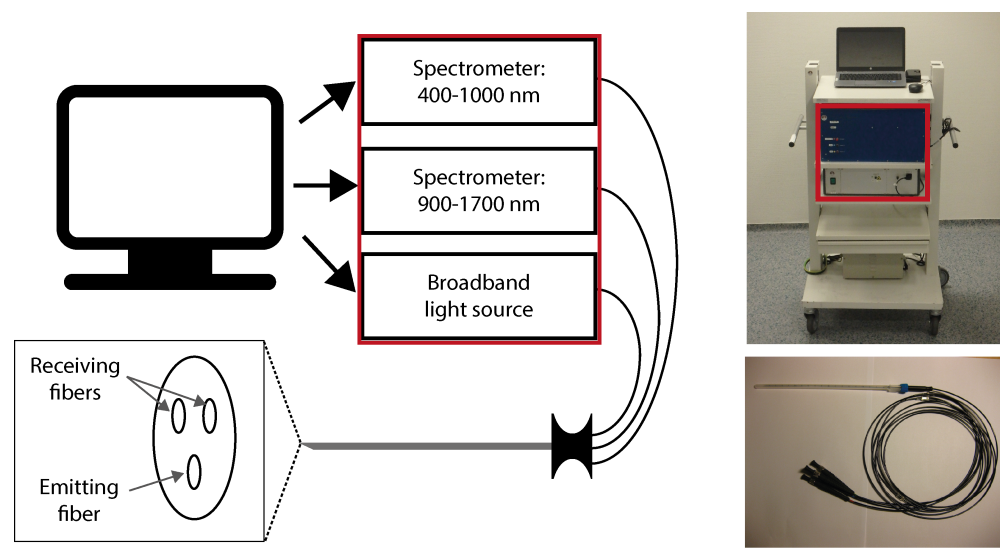

Figure 5.2: Measurement system On the left a schematic image is shown of the system used to perform the measurements. The system consists of two spectrometers and a broadband light source, which are all controlled by a computer. Measurements are performed using a needle which includes three fibers. One that transports the light from the broadband light source to the tissue (emitting fiber) and two to transport the light from the tissue to the two spectrometers (receiving fibers). The distance between the receiving and emitting fibers is $1.29 \mathrm{~mm}$. On the right images are shown of the system as used during surgery (top image) and the needle used to perform the measurements with (bottom image).

The measurements were performed using clinical-grade disposable $16 \mathrm{G}$ needles (INVIVO, Gainesville, Florida). In the needles, three optic fibers (core diameter = $200 \mu \mathrm{m}$ ) were embedded: one fiber to illuminate the tissue and two fibers to transport the light from the tissue to the two spectrometers. The center to center distances between the emitting and receiving fibers was $1.29 \mathrm{~mm}$. The distance between the emitting and receiving fibers determines the measurement depth, which is approximately the same as the distance between the fibers [24]. 


\subsubsection{Study protocol}

This in vivo study was performed under approval from the internal review board of The Netherlands Cancer Institute (Dutch Trail Register NTR5315). Patients from the Netherlands Cancer Institute, were included, based on preoperative imaging. Patients were selected for inclusion when preoperative imaging indicated colorectal cancer stage CT3 or cT4, and patients would undergo open surgery for tumor removal. All included patients signed informed consent. All ethical guidelines were followed.

During surgery, the surgeon was asked to acquire DRS measurements of healthy fat, healthy colorectal wall and tumor tissue. To obtain the spectra, a probe was placed by the surgeon in contact with the tissue. For tumor measurements, three locations were measured on the surface of the bowel wall which were classified by the surgeon as most suspect for tumor. The surgeons were asked to indicate how certain he or she was that these measurements contained tumor, which was noted. Fat and healthy colorectal wall were measured at a distance from the tumor, to ensure these locations were actually healthy. Per location, the measurement was repeated three times. All locations were marked with a suture. If the surgeon was unable to localize tumor close to or at the surface of the bowel wall no measurements were performed and the patient was excluded from any further analysis. After surgery, the sutures marking the measurement locations were removed and replaced by ink which was visible during microscopic inspection. Subsequently, the specimen was brought to the pathology department and was further processed according to standard protocol. All measured and marked locations were included in hematoxylin-eosin (H\&E) coupes.

\subsubsection{Pathology classification}

Histopathological validation of the DRS measurements was performed by an experienced colorectal pathologist. To this end, the H\&E coupes were examined, under a microscope, and the different tissue types observed in the H\&E coupe were labeled as fat, healthy colorectal wall or tumor. Subsequently, the labeled tissue types were correlated to the DRS measurements which on their turn were classified as fat, healthy colorectal wall or tumor measurements. A measurement was classified as tumor when tumor was present within $1.5 \mathrm{~mm}$ from the surface. For some tumor measurements correlation with histopathology analysis was inconclusive. Correlation with histopathology was classified as inconclusive if tu- 
mor was present on the H\&E coupe over a length of less than $0.5 \mathrm{~mm}$, within 1.5 $\mathrm{mm}$ from the measurement surface. For such small tumor areas correlation with histopathology was too inaccurate to conclude whether tumor was measured or not. To reduce the influence of these measurements on the classification, these were removed from the dataset.

\subsubsection{Data analysis}

Data analysis was performed using Matlab (version 8.5, MathWorks Inc., Natick, Massachusetts). First, all spectra were calibrated using a white reference and dark reference taken before the measurements of each patient [23]. Before classification, all spectra were normalized at $800 \mathrm{~nm}$. Using the entire spectra, two linear support vector machines (SVM) were trained using a tenfold cross-validation to distinguish the three tissue types; fat, healthy colorectal wall and tumor. An SVM is a machine learning technique and a binary classifier, able to distinguish two different classes at once. The first SVM was a one versus all classification to distinguish fat from healthy colorectal wall and tumor. The second SVM was used to separate healthy colorectal wall from tumor. For the training of the first SVM, healthy colorectal wall and tumor were merged into one class. The train dataset of the second SVM only included healthy colorectal wall and tumor spectra. For testing of both SVMs the result of the first SVM determined whether the spectrum was given as an input to the second SVM. Spectra that were not classified as fat were also classified by the second SVM to distinguish between healthy colorectal wall and tumor tissue (Fig. 5.3). The tenfold cross-validated training and testing of both SVMs was repeated ten times to ensure representative results.

To evaluate the classification results the Matthews Correlation Coefficient (MCC) (Equation 5.1) was used together with the accuracy, sensitivity and specificity. The MCC was used because it is less influenced by imbalanced data compared to the accuracy value. The MCC gives a value between -1 and 1 , where -1 stands for complete reverse classification by the classifier, +1 for a perfect classification by the classifier and 0 for no better than random classification by the classifier. In Equation 5.1 TN, TP, FN and FP are the number of true negatives, true positives, false negatives and false positives respectively.

$$
M C C=\frac{T P \times T N-F P \times F N}{\sqrt{(T P+F P)(T P+F N)(T N+F P)(T N+F N)}}
$$




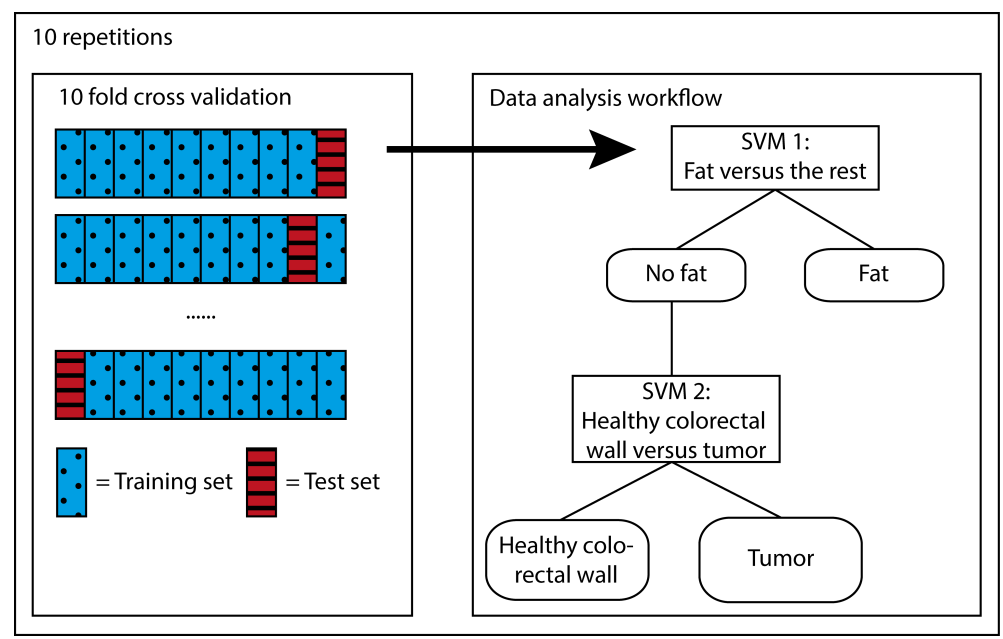

Figure 5.3: Data analysis workflow

\subsubsection{Depth analysis}

With a distance of $1.29 \mathrm{~mm}$ between the emitting and receiving fibers, tumor could be detected up to $1-1.5 \mathrm{~mm}$ in depth [25]. Therefore, measurements were classified as tumor when tumor was present within $1.5 \mathrm{~mm}$ from the measure surface. The influence of the depth of the tumor on the classification result was analyzed as well. This was done by increasing the maximum distance, for a measurement to be classified as tumor, between the measurement surface and the first encountered tumor tissue (Fig. 5.4). The distance was increased from 0 $\mathrm{mm}$ to more than $4 \mathrm{~mm}$. For each distance the classification was re-trained and re-tested and accuracies and MCC values were obtained.

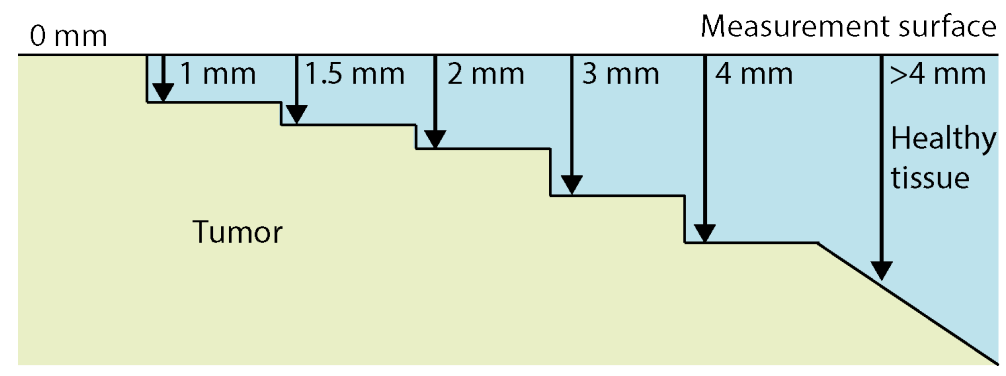

Figure 5.4: The maximum distance between the measurement surface and tumor for a measurement to be classified as tumor. 


\subsubsection{Comparison to clinical judgement}

To determine the added value of the DRS technique to the clinical judgement of the surgeon, results of the classification of the DRS measurements were compared to the tissue classification given by the surgeon. Most added value is obtained on locations of which the surgeon is uncertain whether tumor is present or not. Therefore, locations of which the surgeon indicated not to be sure about the presence of tumor were evaluated separately. To avoid positive resection margins the number of false negative classified locations, the number of locations classified as healthy tissue that were actually tumor, should be zero. To avoid false negative classifications, a new threshold for the classifier to classify a location as tumor had to be determined. For objective evaluation of the uncertain locations, this threshold was determined based on the locations of which the surgeon was certain. Thereafter, this threshold was applied to the classification of the uncertain locations and the results were compared to the judgement of the surgeons.

\subsection{Results}

\subsubsection{Inclusion}

In total, 52 patients were included in the study. Patient and tumor characteristics of the included and measured patients are described in Table 5.1. Eventually, 20 patients were not measured. Four patients were not measured because during surgery, the surgeon was not able to visualize tumor at the bowel surface, of which 2 patients were staged pT4, 1 pT3 and 1 pT0. The other 15 patients were not measured because of logistical reasons. Logistical issues included; surgery that was performed in another hospital, theatre time did not allow additional time for measurements, patients had too extensive disease because of which no resection was performed and therefore no pathology evaluation of the measurement was possible, and changes in the operation room schedule. Of the included patients with rectal cancer, four received neoadjuvant radiotherapy, three in combination with chemotherapy. One patient received neoadjuvant chemotherapy only.

In total, 835 spectra (from 270 locations) were acquired, 402 on fat, 282 on healthy colorectal wall, and 151 on tumor. Histopathology was not available for the tumor locations of one of the patients, therefore 9 spectra were excluded for further analysis. After removal of all tumor measurements with inconclusive histopathology 
Table 5.1: Patient and tumor characteristics

\begin{tabular}{|c|c|c|c|}
\hline & & Included & Measured \\
\hline \multicolumn{2}{|l|}{ Total number of patients } & 52 & 32 \\
\hline \multirow[b]{2}{*}{ Gender } & Male & 29 & 19 \\
\hline & Female & 23 & 13 \\
\hline \multirow[b]{2}{*}{ Age } & Median & 59 & 61 \\
\hline & Interquartile range & $50-68$ & $50-68$ \\
\hline \multirow{5}{*}{ Tumor location } & Appendix & 1 & 0 \\
\hline & Cecum & 7 & 2 \\
\hline & Colon & 24 & 17 \\
\hline & Sigmoid & 13 & 8 \\
\hline & Rectum & 7 & 5 \\
\hline \multirow{6}{*}{ Stage after histopathology ${ }^{\star}$ evaluation } & pT0 & 2 & 1 \\
\hline & pT1 & 0 & 0 \\
\hline & pT2 & 2 & 2 \\
\hline & pT3 & 22 & 14 \\
\hline & pT4 & 24 & 13 \\
\hline & Recurrence & 2 & 2 \\
\hline \multirow{5}{*}{ Exclusion } & No tumor at surface & 4 & - \\
\hline & Surgery at another hospital & 1 & - \\
\hline & Theater time & 2 & - \\
\hline & Too extensive disease & 6 & - \\
\hline & Changes in schedule & 7 & - \\
\hline
\end{tabular}

*T stages include staging after pathological evaluation. 
(Fig. 5.5b), 87 tumor measurements were left. For fat and healthy colorectal wall, most measured locations consisted entirely of fat or healthy colorectal wall, respectively. In Figure 5.6 the mean spectra of fat, healthy colorectal wall and tumor are shown, the spectra are normalized at $800 \mathrm{~nm}$.

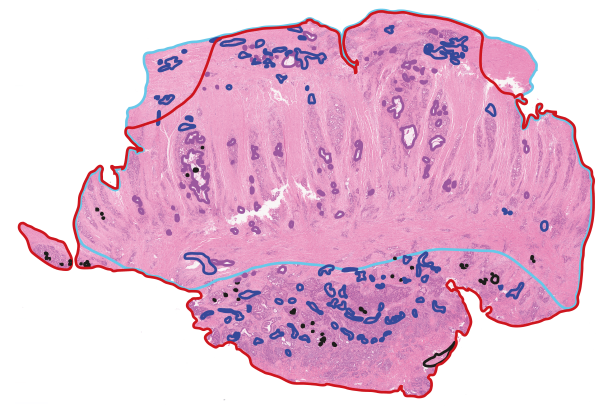

(a) Included

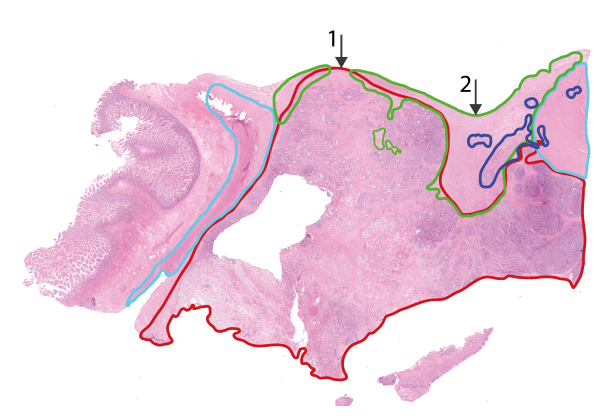

(b) Excluded

Figure 5.5: H\&E slides of a measured locations with conclusive and inconclusive correlation to histopathology. H\&E slides were annotated by a pathologist. Red = tumor, light blue = muscle, green = fibrosis, dark blue = inflammation. A) Conclusive histopathology, with a large area of only tumor at the surface. B) Inconclusive histopathology, if the measurement would have been on location 1 , it would be a tumor measurement, however on location 2, less than $0.5 \mathrm{~mm}$ to the right it would be a fibrosis measurement. Locations with histopathology similar to B were excluded whereas locations with histopathology similar to A were used for classification.

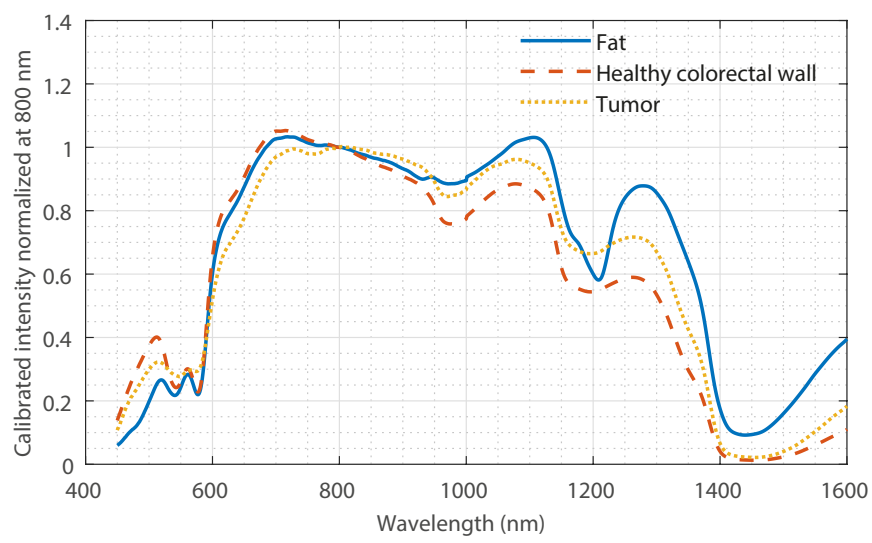

Figure 5.6: Mean spectra of fat, healthy colorectal wall and tumor, normalized at $800 \mathrm{~nm}$. 


\subsubsection{Classification results}

The evaluation of the classification was performed per tissue type, all values were calculated as a one versus all classification. Classification of fat was done with a mean MCC of 0.83 , classification healthy colorectal wall with a mean MCC of 0.77 and tumor with a mean MCC of 0.73 . In Table 5.2 the mean accuracy, MCC, sensitivity and specificity values are shown for all tissue types. In Figure 5.7 the ROC curves of each tissue type are shown. For each tissue type, one iteration of the ten repetitions is shown. The average accuracy over all tissue types, weighted based on the number of measurements per tissue type, was 0.91 .

Table 5.2: Mean values (STD) of accuracy, MCC, sensitivity and specificity, per tissue type

\begin{tabular}{lcccc}
\hline Tissue type & Accuracy & MCC & Sensitivity & Specificity \\
\hline Fat & $0.92(0.00)$ & $0.83(0.01)$ & $0.89(0.01)$ & $0.94(0.00)$ \\
Healthy colorectal wall & $0.89(0.01)$ & $0.77(0.01)$ & $0.92(0.01)$ & $0.87(0.01)$ \\
Tumor & $0.94(0.00)$ & $0.73(0.02)$ & $0.90(0.02)$ & $0.94(0.00)$ \\
\hline
\end{tabular}

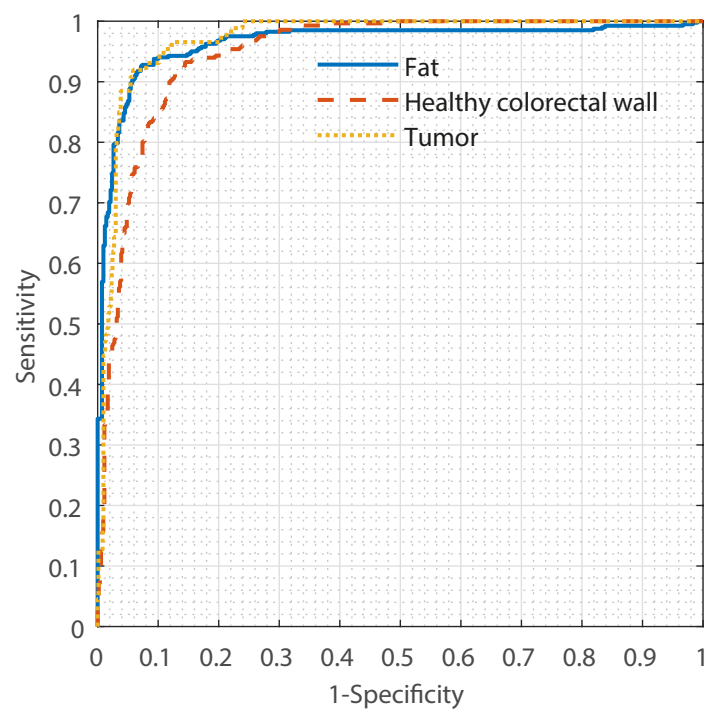

Figure 5.7: ROC curves of one iteration for all three tissue types. 


\subsubsection{Depth analysis}

To examine the influence of the depth of the tumor, the distance between the measurement surface and the first encountered tumor tissue, was varied. The first step was to include only measurements with tumor at the surface, so at $0 \mathrm{~mm}$ in depth. From this a $1 \mathrm{~mm}$ increase in depth was taken. The depth of $1.5 \mathrm{~mm}$ was included as well, because this distance was used in the original analysis. In Figure 5.8 the resulting accuracies and MCC values for the different depth are shown for tumor. Both the accuracy and MCC show an optimum around 1-1.5 $\mathrm{mm}$. Accuracy and MCC decrease if the tumor starts at a depth of $2 \mathrm{~mm}$ or more.

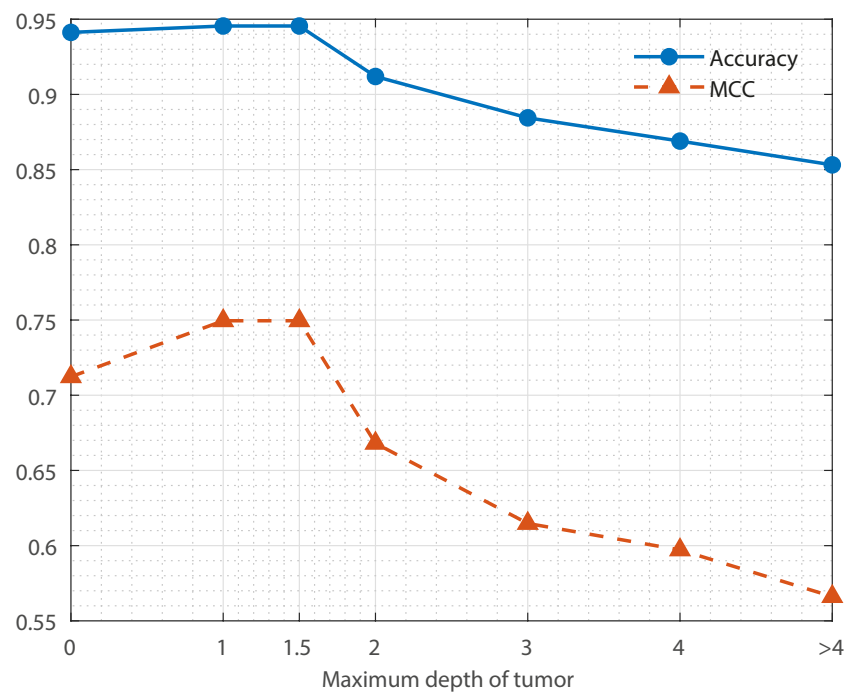

Figure 5.8: The accuracy and MCC values for tumor tissue with an increase in maximum depth for tumor measurements to be classified as tumor.

\subsubsection{Comparison to clinical judgement}

The surgeon indicated not to be sure whether tumor was measured in 54 out of 270 locations. For these locations the technique could be of added value by providing the surgeon with more information about the tissue type. For the analysis of these locations, the threshold of the classifier was adjusted such that no false negative classifications were obtained on the locations of which the surgeon was certain. With this adjusted threshold, the uncertain locations were classi- 
fied. The classification of the uncertain locations resulted in $25 \%$ of the healthy locations falsely classified as tumor and no measurements on tumor tissue classified as healthy tissue. When evaluating the judgement of the surgeons, $69 \%$ of the healthy tissue locations were incorrectly classified as tumor by the surgeon. In Table 5.3, an overview is given on the classification results of the classifier and surgeon compared to the histopathology. Locations are separated between healthy and tumor, where healthy included fat and healthy colorectal wall.

Table 5.3: Confusion matrix of pathology classification and judgement by the surgeon and the classification by the classifier

\begin{tabular}{llcccc}
\hline & & \multicolumn{2}{c}{ Classification by surgeon } & \multicolumn{2}{c}{ Classification by classifier } \\
& & Healthy & Tumor & Healthy & Tumor \\
\hline \multirow{2}{*}{ Pathology } & Healthy & $36(23 \%)$ & $116(73 \%)$ & $127(80 \%)$ & $25(16 \%)$ \\
& Tumor & $0(0 \%)$ & $6(4 \%)$ & $0(0 \%)$ & $6(4 \%)$ \\
\hline
\end{tabular}

\subsection{Discussion}

To the best of our knowledge this is the first in vivo study using DRS to distinguish tumor tissue from healthy surrounding tissues in colorectal cancer surgery. It is shown that tumor tissue can be distinguished from healthy colorectal wall and fat with a sensitivity and specificity of 0.90 and 0.94 , respectively, giving an accuracy of 0.94 .

Previous studies using DRS to discriminate colorectal tumor tissue from healthy surrounding tissue were mainly focused on the application during endoscopy [1418] or were performed ex vivo [19-21]. The endoscopy studies showed a major difference in blood content between tumor and healthy mucosal tissue. In these studies, only visible wavelengths were included in the analysis. As blood is the main absorber in this wavelength range, differences in blood content can reliably be determined. In the current study differentiation between tumor and healthy tissue needs to be made during surgery, were the presence of blood on the measurement surface cannot always be controlled. This makes parameters obtained in the wavelength region of blood absorption less reliable for classification. For this reason, also the near-infrared wavelength range was included, to be able to obtain additional parameters outside of the blood absorption wavelength range [14-18]. Moreover, during endoscopy healthy surrounding tissue only consists of mucosal tissue from the lumen of the colon. During surgery mucosal tissue 
will not be encountered, but fat and bowel muscle tissue will be. Therefore, during the surgical application of DRS, tumor has to be differentiated from fat and muscle tissue, instead of from mucosal tissue like in colonoscopy. Discrimination between fat and tumor tissue seems an easy task [20]. As shown by the MCC values in Table 5.2 the separation of healthy colorectal wall and tumor tissue is more difficult.

When comparing the current study to the results obtained previously in the ex vivo studies, the accuracies seem similar, ranging from $91 \%$ to $99 \%$ [19-21]. If the imbalance in the current in vivo dataset is taken into account and a weighted average is taken for all three tissue types, an accuracy of 0.91 for the current in vivo study is obtained. In the ex vivo study an average accuracy over all tissue types of 0.95 was found, which is slightly higher [20]. The main reason for the difference in accuracies is the less controlled measurement environment for the current in vivo study. This will lead to less accurate correlation with histopathology for the evaluation of the classification, which will lead to a decrease in accuracy. In Table 5.2 the results are shown for the classification in which only conclusive histopathology was included. If all measured locations are included, including the ones of which histopathology classification was inconclusive (Fig. 5.5b), the MCC values of healthy colorectal wall and tumor show a decrease to 0.67 and 0.56 respectively. Which indeed shows that uncertainty in the histopathology correlation will influence the outcome of the classification. This problem is hard to circumvent. One way to get a more reliable accuracy on the differentiation between healthy colorectal wall and tumor is to increase the number of included patients. With an increase in the number of patients, at least the number of patients with clear histopathology will increase and potentially also the ratio with the number of patients with unclear histopathology..

Furthermore, during the ex vivo studies it is simple to obtain measurement locations with pure tissue types. In the current study this was not always possible, because tumor did not always penetrate the bowel wall. Therefore, some of the tumor measurements were performed with a small layer of healthy colorectal wall between the measurement surface and tumor. If the maximum depth of tumor from the measurement surface was increased from 0 up to more than $4 \mathrm{~mm}$, a drop in accuracy and MCC value for tumor is shown for depth of tumor more than $1.5 \mathrm{~mm}$ (Fig. 5.8). This is most likely due to the small amount or absence of tumor present in the measured volume. Therefore, classification of these measurements is harder or even impossible. The measurement volume is mainly determined by 
the distance between the emitting and receiving fibers. In the current study the fibers were $1.29 \mathrm{~mm}$ apart resulting in a measurement depth of approximately 1 $1.5 \mathrm{~mm}$. If this distance is increased the measurement volume will increase and with this the depth until which tumor can be detected. Therefore, with an increase in distance between the receiving and emitting fibers, the accuracy of tumor detection at larger depths will be better. The decrease in accuracy and MCC value for measurements with tumor at the surface $(0 \mathrm{~mm})$, is most likely due to the low number of measurements in this group.

Since clinically a tumor free margin (CRM) is defined as $>2 \mathrm{~mm}$, correct classification of measurements with a maximum depth of $2 \mathrm{~mm}$ will be more useful for the surgeon than a classification which includes also tumor tissue deeper than $2 \mathrm{~mm}$. The current technique will provide an average of the tissue types in the entire measurement volume. Therefore, if the volume is up to $2 \mathrm{~mm}$ in depth, the surgeon can act on the information provided by the technique, because if tumor is indicated by the technique, tumor will be present within $2 \mathrm{~mm}$ from the resection margin, resulting in a positive CRM. Whereas, if it would provide information from further than $2 \mathrm{~mm}$ in depth it would be hard for the surgeon to determine whether to act on it or not. Since the current technique cannot locate the depth of the tumor, tumor could still be more than $2 \mathrm{~mm}$ from the resected surface, resulting in a negative CRM, but it could also be within $2 \mathrm{~mm}$ from the resected surface where it will cause a positive CRM.

In this study four patients were excluded because the surgeon indicated that no tumor could be measured. No measurements were performed in these patients to ensure a sufficient tumor to healthy measurement ratio for further classification. Of these four excluded patients, two tumors were staged by pathology as pT4, one as PT3 and one as pT0. The patients with pT4 staged tumors received neoadjuvant chemotherapy and showed a significant inflammatory reaction around the tumor area. It would have been possible to measure tumor at the surface or close to the surface of the bowel wall of these two patients. However, the surgeon was unable to distinguish tumor from inflammation. Therefore, the surgeon performed a more extensive resection to prevent positive margins. Due to the more extensive surgery, the surgeon was unable to perform measurement close to the tumor. These typical cases, where the surgeon was unable to discriminate tumor tissue from healthy tissue and therefore extended the resection, illustrate once again the need for a technique that can real-time classify tissue during surgery.

When the threshold of the classification is set such that the classification will not 
give any false negative predictions, the added value of the technique is shown for the uncertain locations (Table 5.3). For these locations the surgeon indicated not to be sure whether there was tumor present or not. We defined our threshold such that no false negative predictions were allowed, so no tumor locations should be classified as healthy, avoiding positive resection margins. As shown in Table 5.3 the DRS technique causes a large decrease in the number false positive classified locations compared to clinical judgement. Classification of the DRS measurements resulted in $25 \%$ of the locations wrongly classified as tumor, instead of $69 \%$ of the locations wrongly indicated as tumor by the surgeon. The specificity of $75 \%$ (Table 5.3) is lower than the 0.94 as shown in Table 5.2, this is due to defining the sensitivity at 1.00 , which inevitably results in a decrease of the specificity. However, even by setting the sensitivity at 1.00 we observed a significant decrease in false positive classified locations with DRS compared to the surgeon. From these results is can be concluded that DRS can potentially result in less extensive surgery and thereby might lead to less complications during and after surgery.

The translation of DRS into clinical practice has taken some time. With this study a major step is taken to introduce DRS in the clinic. This study shows that it is possible to use DRS during colorectal cancer surgery to discriminate healthy tissue from tumor tissue. Further development of the technology could lead to incorporation of the technology in a smart surgical tool. When the technique is used in the form of a hyperspectral camera it is also possible to incorporate it into a laparoscopic camera or during robotic surgery.

\subsection{Conclusion}

In this in vivo study, tumor can be classified with an accuracy of 0.94 and a sensitivity and specificity of 0.90 and 0.94 , respectively. The false positive rates from the judgement of the surgeon and the false positive rate of the classification of the DRS spectra, demonstrate the potential of using DRS in vivo in colorectal cancer. Future research should focus on making the analysis of the obtained spectra realtime, to enable in vivo evaluation of the technology. 


\section{References}

[1] F. Bray et al., "Global cancer statistics 2018: GLOBOCAN estimates of incidence and mortality worldwide for 36 cancers in 185 countries," CA: A Cancer Journal for Clinicians, vol. 68, pp. 394424, nov 2018.

[2] R. L. Siegel et al., "Colorectal cancer statistics, 2017," CA: a cancer journal for clinicians, vol. 67, no. 3, pp. 177-193, 2017.

[3] K. D. Miller et al., "Cancer treatment and survivorship statistics, 2016," CA: A Cancer Journal for Clinicians, vol. 66, pp. 271-289, jun 2016.

[4] M. M. Lange and C. J. H. van de Velde, "Long-term anorectal and urogenital dysfunction after rectal cancer treatment," Seminars in Colon and Rectal Surgery, vol. 21, no. 2, pp. 87-94, 2010.

[5] S. E. Delacroix and J. C. Winters, "Voiding Dysfunction after Pelvic Colorectal Surgery," Clinics in Colon and Rectal Surgery, vol. 23, no. 2, pp. 119-127, 2010.

[6] J. S. Park et al., "A Circumferential Resection Margin of $1 \mathrm{~mm}$ Is a Negative Prognostic Factor in Rectal Cancer Patients With and Without Neoadjuvant Chemoradiotherapy," Diseases of the Colon \& Rectum, vol. 57, no. 8, 2014.

[7] I. D. Nagtegaal and P. Quirke, "What Is the Role for the Circumferential Margin in the Modern Treatment of Rectal Cancer?," Journal of Clinical Oncology, vol. 26, no. 2, pp. 303-312, 2008.

[8] D. J. Evers et al., "Optical sensing for tumor detection in the liver," European Journal of Surgical Oncology, vol. 39, no. 1, pp. 68-75, 2013.

[9] E. Tanis et al., "In vivo tumor identification of colorectal liver metastases with diffuse reflectance and fluorescence spectroscopy," Lasers in Surgery and Medicine, vol. 48, pp. 820-827, nov 2016.

[10] J. W. Spliethoff et al., "Improved identification of peripheral lung tumors by using diffuse reflectance and fluorescence spectroscopy," Lung Cancer, vol. 80, no. 2, pp. 165-171, 2013.

[11] L. L. Boer et al., "Fat/water ratios measured with diffuse reflectance spectroscopy to detect breast tumor boundaries," Breast Cancer Research and Treatment, vol. 152, no. 3, pp. 509-518, 2015.

[12] J. S. Soares et al., "Diagnostic power of diffuse reflectance spectroscopy for targeted detection of breast lesions with microcalcifications," Proceedings of the National Academy of Sciences, vol. 110, pp. 471 LP - 476, jan 2013.

[13] S. G. Brouwer de Koning et al., "Toward complete oral cavity cancer resection using a handheld diffuse reflectance spectroscopy probe," Journal of Biomedical Optics, vol. 23, pp. 1-9, oct 2018.

[14] G. Zonios et al., "Diffuse reflectance spectroscopy of human adenomatous colon polyps in vivo," Applied optics, vol. 38, no. 31, pp. 6628-6637, 1999.

[15] H.-W. Wang et al., "Diffuse reflectance spectroscopy detects increased hemoglobin concentration and decreased oxygenation during colon carcinogenesis from normal to malignant tumors.," Optics express, vol. 17, no. 4, pp. 2805-17, 2009. 
[16] H. K. Roy et al., "Spectroscopic Microvascular Blood Detection From the Endoscopically Normal Colonic Mucosa: Biomarker for Neoplasia Risk," Gastroenterology, vol. 135, no. 4, pp. 10691078, 2008.

[17] A. Dhar et al., "Elastic scattering spectroscopy for the diagnosis of colonic lesions: initial results of a novel optical biopsy technique," Gastrointestinal Endoscopy, vol. 63, pp. 257-261, feb 2006.

[18] J. R Mourant et al., Elastic scattering spectroscopy as a diagnostic tool for differentiating pathologies in the gastrointestinal tract: Preliminary testing, vol. 1. apr 1996.

[19] G. C. Langhout et al., "Differentiation of healthy and malignant tissue in colon cancer patients using optical spectroscopy: A tool for image-guided surgery," Lasers in Surgery and Medicine, vol. 47, no. 7, pp. 559-565, 2015.

[20] E. J. M. Baltussen et al., "Diffuse reflectance spectroscopy as a tool for real-time tissue assessment during colorectal cancer surgery," Journal of biomedical optics, vol. 22, no. 10, pp. 106014106016, 2017.

[21] R. M. Schols, P. Dunias, F. P. Wieringa, and L. P. S. Stassen, "Multispectral characterization of tissues encountered during laparoscopic colorectal surgery," Medical Engineering and Physics, vol. 35, no. 7, pp. 1044-1050, 2013.

[22] R. Nachabé et al., "Estimation of biological chromophores using diffuse optical spectroscopy: benefit of extending the UV-VIS wavelength range to include 1000 to $1600 \mathrm{~nm}$," Biomedical Optics Express, vol. 1, no. 5, pp. 1432-1442, 2010.

[23] R. Nachabe et al., "Estimation of lipid and water concentrations in scattering media with diffuse optical spectroscopy from 900 to $1600 \mathrm{~nm}$," vol. 15, pp. 37010-37015, 2010.

[24] A. J. Gomes and V. Backman, "Algorithm for automated selection of application-specific fiberoptic reflectance probes," Journal of Biomedical Optics, vol. 18, p. 27012, feb 2013.

[25] R. Hennessy et al., "Effect of probe geometry and optical properties on the sampling depth for diffuse reflectance spectroscopy," Journal of biomedical optics, vol. 19, p. 107002, oct 2014. 


\section{Chapter 6}

\section{Comparing in vivo and ex vivo fiberoptic diffuse reflectance spectroscopy in colorectal cancer}

Elisabeth J.M. Baltussen, Susan G. Brouwer de Koning, Katarzyna Jozwiak, Henricus J.C.M. Sterenborg, Theo J.M. Ruers, Translational Biophotonics, 2019 


\section{Abstract}

In vivo data acquisition using fiberoptic diffuse reflectance spectroscopy (DRS) is more complicated and less controlled compared to ex vivo data acquisition. It would be of great benefit if classifiers for in vivo tissue discrimination based on DRS could be trained on data obtained ex vivo. In this study, in vivo and ex vivo DRS measurements are obtained during colorectal cancer surgery. A mixed model statistical analysis is used to examine the differences between the two datasets. Furthermore, classifiers are trained and tested using in vivo and ex vivo data. It is found that with a classifier trained on ex vivo data and tested on in vivo data similar results are obtained compared to a classifier trained and tested on in vivo data. In conclusion, under the conditions used in this study classifiers intended for in vivo tissue discrimination can be trained on ex vivo data. 


\subsection{Introduction}

Colorectal cancer is the third most common cancer worldwide for men and women combined and the second cause of cancer related death [1]. Standard of care for advanced stage colorectal cancer is surgery, which is sometimes combined with neoadjuvant chemo- and/or radiotherapy. In colorectal cancer surgery there are two main challenges. First, complete removal of the tumor, as a positive resection margin is a negative independent predictor of survival and local recurrence [2, 3]. Second, avoiding too extensive surgery to prevent complications from damage to vital structures. Technology for intraoperative tissue classification could be of great benefit to decrease the number of positive resection margins, while preventing complications due to too extensive surgery.

Fiberoptic diffuse reflectance spectroscopy (DRS) can be used for intraoperative tissue classification. In DRS, light over a broad wavelength range is send through an optical fiber into the tissue. Within the tissue, the light will undergo scattering and absorption, which depends on tissue characteristics and varies with the wavelength of the light $[4,5]$. Part of the light will be scattered back to the surface of the tissue where it can be collected with a second fiber. Based on the collected spectrum different tissue types can be distinguished [5].

DRS has been used before for tissue classification in cancers, like breast, head and neck, liver, lung, and also colorectal cancer [6-12]. In colorectal cancer, most research was done during endoscopy to detect tumor tissue inside the lumen, where only mucosal tissue and tumor tissue can be encountered [13-18]. However, during surgery tissue is assessed from outside the lumen, where no mucosal tissue but manly fat and healthy colorectal wall are present. This makes classification during surgery different compared to the endoscopic setting. Some studies have been focused on the use of DRS during colorectal cancer surgery, with accuracies ranging from $91-95 \%$. However, all these studies were performed ex vivo $[9,10,12]$. In order to use DRS during surgery, in vivo use of DRS has to be evaluated as well. Data acquisition in vivo during colorectal cancer surgery is more complicated and less controlled compared to the ex vivo setting, in terms of pressure applied on the probe, correlation with pathology and ambient light control. Therefore, it would be of great benefit if data obtained ex vivo could be used reliably to train a classifier intended for in vivo use.

So far, not many studies have focused on the question whether results obtained ex vivo can be used for in vivo application. One study on mouse ear models was 
done in which DRS data was obtained in living mice (in vivo), 5-10 min after excision (ex vivo) of tissue and after 24 and 72 hours of storage [18]. Furthermore, a study was done on human nerves during surgery and post mortem [19]. Both studies found differences between in vivo measurements and ex vivo measurements. However, both studies were focused on ex vivo measurements after long term storage. Therefore, in the current study, in vivo and ex vivo measurements are performed on colorectal cancer specimen during surgery and within one hour after resection. Measurement locations were marked in vivo to direct the ex vivo measurements and to allow accurate pathology registration. A mixed-effect linear regression is done to compare the results obtained in vivo and ex vivo. Furthermore, a classifier is trained using the ex vivo data and tested on the in vivo data to examine if a similar accuracy is obtained compared to the classification trained and tested on in vivo data.

\subsection{Materials and methods}

\subsubsection{Measurement set-up}

The DRS system used for this study consisted of two spectrometers and Tungsten halogen light source with embedded shutter. One spectrometers resolves light in the visual wavelength range, 400-1100 nm (Andor Technology, DU420ABRDD), the other one resolves light in the near-infrared wavelength range, 900-1700 nm (Andor Technology, DU492A-1.7). The light source emits light from 360-2500 $\mathrm{nm}$. The system is controlled by custom made LabView software (National Instruments, Austin, Texas). A detailed description of the calibration of the system can be found elsewhere [20, 21].

Measurements were performed using clinical-grade disposable $16 \mathrm{G}$ needles (INVIVO, Gainesville, Florida). Three optical fibers with a core diameter of $200 \mu \mathrm{m}$ were embedded in the needle, one to transport the light from the source to the tissue and two to transport the light from the tissue to the two spectrometers. The center to center distance between the delivering fiber and the two collecting fibers was $1.29 \mathrm{~mm}$ (Fig. 6.1).

\subsubsection{Data acquisition}

This study was performed under approval from the internal review board (Dutch Trail Register NTR5315). Patients with colorectal cancer, who had to undergo 


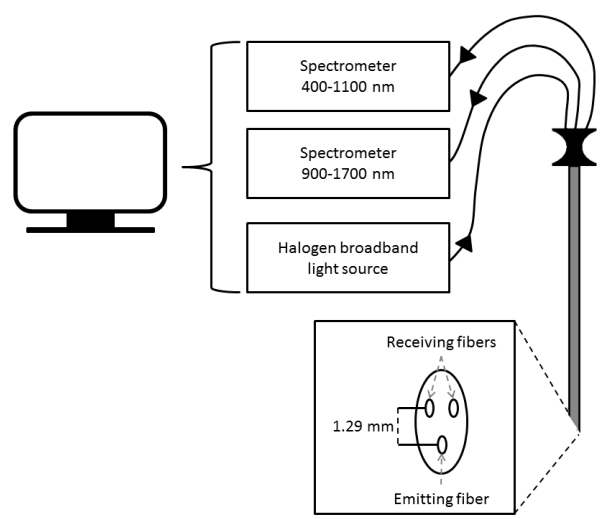

Figure 6.1: Schematic of the measurement set-up. The set-up includes two spectrometers, and a halogen broadband light source. The measurement needle contains three fibers, one to transport light to the tissue and two to transport the light from the tissue to the two spectrometers.

open surgery to remove the tumor, in the Netherlands Cancer Institute, were included. All patients were included based on preoperative imaging, which indicated advanced stage colorectal cancer, stage T3 or T4. All included patients signed informed consent. All ethical guidelines were followed.

The surgeon was asked to perform measurements during surgery by placing the needle on healthy fat, healthy colorectal wall and tumor. All measurement locations were marked with a suture. After resection, the measurements were repeated ex vivo on the marked locations. These measurements were performed using the same needle that was used in vivo. After the ex vivo measurements the sutures were removed and ink was used to mark the measurement locations. Thereafter, the specimen was brought to the Pathology Department where the specimen was processed according to standard protocol. After fixation, pathology slides were obtained of all measured locations. These pathology slides were annotated by a pathologist to obtain a ground truth for all measured locations.

\subsubsection{Data processing}

The spectra obtained from the two spectrometers were stitched together before performing a single parameter fit using an analytical model, based on optical diffusion theory, to obtain tissue constituents and optical properties of the measured tissue volume [20]. This model uses known absorption spectra and scattering characteristics of constituents present in the measured volume to fit a spectrum to the measured reflectance and generates estimations of the tissue constituents 
and scattering characteristics. The model has been described in detail elsewhere $[4,20]$. For this study a model with 12 parameters was used, four focused on blood, two on water and fat, three parameters were focused on scattering properties and a single parameter for collagen, beta-carotene and a scale factor Table 6.1. The scale factor couples the incident collimated light to the diffuse field in the tissue and is related to the scattering phase function [4]. The model was fitted on the measured spectra using a Levenberg-Marquardt least squares minimization method. Based on the confidence intervals obtained for all fitted parameters and based on the deviation between the measured spectrum and the obtained fit, using the relative residual, bad fits were excluded from the analysis. The relative residual was computed by dividing the absolute difference between the measured spectrum and obtained fit by the mean of the measured spectrum. If the relative residual was above 0.9 the fit was excluded. The analytical model enabled the detection of possible differences between in vivo and ex vivo to biological parameters.

Table 6.1: All parameters used in analytical model, grouped.

\begin{tabular}{|c|c|c|}
\hline Group & Parameter & Abbreviation used \\
\hline Blood & $\begin{array}{l}\text { Volume fraction of blood present in the tissue } \\
\text { Saturation of the blood } \\
\text { Amount of methemoglobin present } \\
\text { Diameter of the blood vessels }\end{array}$ & $\begin{array}{l}\text { Blood }(\%) \\
\text { StO2(\%) } \\
\text { Methemoglobin } \\
\text { Diameter of blood vessels }\end{array}$ \\
\hline Water and fat & $\begin{array}{l}\text { Volume fraction of water plus fat } \\
\text { Volume fraction of fat from water plus fat fraction }\end{array}$ & $\begin{array}{l}\text { Water + Fat (\%) } \\
\text { Fat/(Water + Fat) (\%) }\end{array}$ \\
\hline Scattering & $\begin{array}{l}\text { Scattering coefficient at } 800 \mathrm{~nm} \\
\text { Scattering wavelength slope } \\
\text { Fraction of Mie over Rayleigh scattering }\end{array}$ & $\begin{array}{l}\text { Scattering at } 800 \mathrm{~nm} \\
\text { Scattering slope } \\
\text { Fraction of Mie scattering } \\
\text { over Rayleigh scattering }\end{array}$ \\
\hline Single parameters & $\begin{array}{l}\text { Collagen } \\
\text { Beta-carotene } \\
\text { Scale factor }\end{array}$ & $\begin{array}{l}\text { Collagen } \\
\text { Beta-carotene } \\
\text { Scale factor }\end{array}$ \\
\hline
\end{tabular}

\subsubsection{Statistics}

A mixed-effect linear regression was performed to compare fitted parameters between in vivo data and ex vivo measurements, and between tissue type measurements. A cross-classified data structure (Fig. 6.2) was taken into account to model correlation between all measurements performed within one patient in the different tissue type and measurement type. 


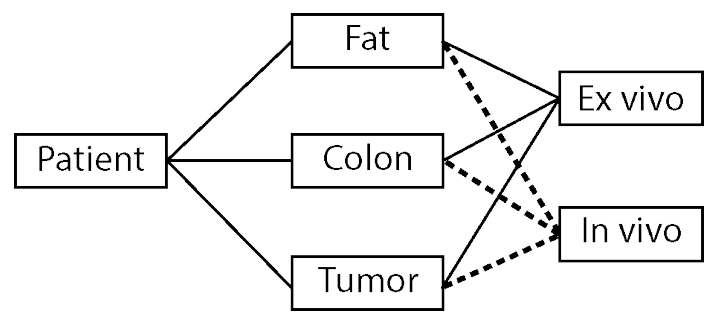

Figure 6.2: Cross-classified data structure. A comparison between ex vivo and in vivo is made for each tissue type on a per patient basis. The lines indicate correlations between the measurements.

Separate regression models were fitted to compare in vivo and ex vivo measurements within each tissue type. Furthermore, comparisons between tumor and fat, and tumor and healthy colorectal wall were performed, for the in vivo and ex vivo measurements, separately. All $p$-values of 0.05 or lower were considered significant.

\subsubsection{Classification}

Classification of the measurements was performed using a linear support vector machine (SVM). Due to the multiclass database, three binary SVMs were used to obtain a complete classification of the dataset. The three binary classifiers included one for fat versus healthy colorectal wall, one for fat versus tumor and one for healthy colorectal wall versus tumor. The SVM was trained and tested using a leave one patient out (LOPO) cross-validation. Four different train and test sets were used to train and test the SVM (Table 6.2).

Table 6.2: Overview of the four different combinations of train and test sets

\begin{tabular}{ll}
\hline Train dataset & Test dataset \\
\hline Ex vivo & Ex vivo \\
In vivo & In vivo \\
Ex vivo and in vivo & Ex vivo and in vivo \\
Ex vivo & In vivo \\
\hline
\end{tabular}

In the final classifier, the ex vivo data of the patient of which the in vivo data was used for testing, was left out of the train dataset. All classifications were performed twice, once with all fitted parameters and once with only the selected parameters. Selection of the parameters was done using forward feature selection on only ex vivo data or on only in vivo data or on a combination of in and ex vivo data. The classifications were not optimized, but were only used to compare the difference 
in performance due to different train and test datasets. The performance evaluation was done using the area under the curve (AUC) of the ROC curve and the Matthews correlation coefficient (MCC). The performance of the different classifiers was compared using the McNemars test [22], where a p-value of 0.05 was determined as significant.

\subsection{Results}

\subsubsection{Inclusion}

For this study 28 patients were included, 17 male and 11 female, of 26 patients tissue was measured in vivo and ex vivo. Measurements of one patient could only be performed in vivo, because the needle could not be used anymore after the in vivo measurement. In one patient only ex vivo measurements could be performed due to failure of the system during the in vivo measurements. The median age was 61.5 years with an interquartile range of 52.25-68 years. Most tumors were located in the colon $(n=15)$, followed by the sigmoid $(n=8)$ and the rectum $(n=5)$. In total, 1605 spectra were measured, of which 288 were excluded because a tissue type was measured which was not included in the analysis i.e. fibrosis, inflammation and necrosis $(n=101)$, or because no proper fit was obtained $(n=187)$. In Table 6.3 an overview is given of the 1317 included spectra.

Table 6.3: Overview of all included spectra

\begin{tabular}{lccc}
\hline Tissue type & In vivo & Ex vivo & Total \\
\hline Fat & 321 & 420 & 741 \\
Healthy colorectal wall & 172 & 262 & 434 \\
Tumor & 83 & 59 & 142 \\
\hline Total & 576 & 741 & 1317 \\
\hline
\end{tabular}

\subsubsection{Statistical analysis}

Table 6.4 shows $p$-values for the difference in parameter value between in vivo and ex vivo measurements for all parameters and each tissue type separately. The Fat/(Water + Fat) (\%), the scattering slope, the scale factor and the diameter of the blood vessels showed no significant difference between in and ex vivo in any of the tissue types. For healthy colorectal wall, the scattering at $800 \mathrm{~nm}$, 
the scale factor, the diameter of the blood vessels and the fraction of Mie versus Rayleigh scattering had a $p$-value above 0.05 . Finally, for tumor, the Fat/(Water + Fat) (\%), scattering at $800 \mathrm{~nm}$, scattering slope, scale factor, the diameter of the blood vessels and the amount of beta-carotene did not show a significant difference between in vivo and ex vivo.

Comparisons between tumor and fat, and between tumor and healthy colorectal wall for each parameter, for in vivo and ex vivo separately are shown in 6.5.

For both tumor versus fat and tumor versus healthy colorectal wall, a significant difference was seen in StO2 (\%) for both in vivo and ex vivo. Moreover, for tumor versus fat, a significant difference was found in the Fat/(Water + Fat) (\%) and in the scattering slope, both in and ex vivo. The scattering at $800 \mathrm{~nm}$ was only significantly different in the ex vivo measurements for tumor versus fat. For tumor versus healthy colorectal wall Water + Fat (\%) was significantly different both in and ex vivo and the scattering slope and fraction of Mie over Rayleigh scattering were only significantly different in the in vivo measurements.

Table 6.4: Significance level of the difference between in vivo and ex vivo for all parameters and all tissue types separately, the arrows show the direction of change from in vivo to ex vivo

\begin{tabular}{lllllll}
\hline Parameter & \multicolumn{2}{c}{ P-value fat } & \multicolumn{2}{l}{$\begin{array}{l}\text { P-value } \\
\text { colorectal wall }\end{array}$} & P-value tumor \\
& \multicolumn{3}{l}{ healthy } \\
\hline Blood (\%) & 0.01 & $\uparrow$ & 0.00 & $\uparrow$ & 0.00 & $\uparrow$ \\
StO2 (\%) & 0.00 & $\uparrow$ & 0.00 & $\uparrow$ & 0.00 & $\uparrow$ \\
Water + Fat (\%) & 0.00 & $\downarrow$ & 0.01 & $\downarrow$ & 0.03 & $\downarrow$ \\
Fat/(Water + Fat) (\%) & 0.03 & $\uparrow$ & 0.05 & $\uparrow$ & 0.72 & - \\
Scattering at 800 nm & 0.00 & $\uparrow$ & 0.27 & - & 0.58 & - \\
Scattering slope & 0.56 & - & 0.00 & $\uparrow$ & 0.75 & - \\
Scale factor & 0.30 & - & 0.75 & - & 0.41 & - \\
Diameter of blood vessels & 0.96 & - & 0.44 & - & 0.85 & - \\
Fraction of Mie scattering over & 0.05 & $\downarrow$ & 0.58 & - & 0.01 & $\uparrow$ \\
Rayleigh scattering & & & & & & \\
Collagen & 0.01 & $\uparrow$ & 0.00 & $\uparrow$ & 0.00 & $\uparrow$ \\
Beta-carotene & 0.00 & $\uparrow$ & 0.00 & $\uparrow$ & 0.20 & - \\
Methemoglobin & 0.00 & $\downarrow$ & 0.00 & $\downarrow$ & 0.01 & $\downarrow$ \\
\hline
\end{tabular}

\subsubsection{Selected parameters}

To obtain the most important parameters used in the classification, forward feature selection was performed three times, once on only ex vivo data, once on only in vivo data and finally on the combination of both. Based on the outcome of all three feature selections, four parameters were selected and they all were selected within the first five parameters in all three feature selections. The four parameters 
Table 6.5: Significance level of the difference in parameters between tumor and fat and tumor and healthy colorectal wall, for in vivo and ex vivo separately, the arrows show the direction of change from tumor to fat and from tumor to healthy colorectal wall

\begin{tabular}{lllllllll}
\hline & \multicolumn{3}{l}{ Tumor versus fat } & \multicolumn{5}{l}{$\begin{array}{l}\text { Tumor versus healthy } \\
\text { colorectal wall }\end{array}$} \\
\hline Parameter & \multicolumn{3}{l}{ P-value in vivo } & \multicolumn{3}{c}{ P-value ex vivo } & P-value in vivo & P-value ex vivo \\
\hline Blood (\%) & 0.09 & - & 0.01 & $\downarrow$ & 0.15 & - & 0.34 & - \\
StO2 (\%) & 0.00 & $\uparrow$ & 0.00 & $\uparrow$ & 0.00 & $\uparrow$ & 0.00 & $\uparrow$ \\
Water + Fat (\%) & 0.05 & $\uparrow$ & 0.47 & - & 0.01 & $\uparrow$ & 0.00 & $\uparrow$ \\
Fat/(Water + Fat) (\%) & 0.00 & $\uparrow$ & 0.00 & $\uparrow$ & 0.89 & - & 0.47 & - \\
Scattering at 800 nm & 0.20 & - & 0.00 & $\uparrow$ & 0.06 & $\downarrow$ & 0.70 & - \\
Scattering slope & 0.00 & $\downarrow$ & 0.00 & $\downarrow$ & 0.01 & $\downarrow$ & 0.09 & $\uparrow$ \\
Scale value & 0.81 & - & 0.49 & - & 0.95 & - & 0.20 & - \\
Diameter of blood vessel & 0.09 & - & 0.15 & - & 0.09 & - & 0.32 & - \\
Fraction of Mie scattering & 0.02 & $\uparrow$ & 0.33 & - & 0.03 & $\uparrow$ & 0.70 & $\downarrow$ \\
over Rayleigh scattering & & & & & & & & \\
Collagen & 0.36 & - & 0.28 & - & 0.71 & - & 0.12 & - \\
Beta-carotene & 0.26 & - & 0.49 & - & 0.10 & - & 0.27 & - \\
Methemoglobin & 0.00 & $\uparrow$ & 0.08 & $\uparrow$ & 0.03 & $\uparrow$ & 0.18 & - \\
\hline
\end{tabular}

included Blood (\%), StO2 (\%), Water + Fat (\%) and Fat/(Water + Fat) (\%). The first three parameters all showed significant differences between in vivo and ex vivo measurements for all tissue types (Table 6.4). The fourth parameter did show significant difference between in and ex vivo for fat and healthy colorectal wall but not for tumor. All selected parameters were significantly different between in and ex vivo for almost all tissue types, but the difference between in and ex vivo for all tissue types was in the same direction.

Of the four selected parameters, Blood (\%) only showed a significant difference between tumor and fat ex vivo (Table 6.5). For StO2 (\%) there was a significant difference between tumor and fat and tumor and healthy colorectal wall both in and ex vivo. Furthermore, the difference was in the same direction for in and ex vivo. Water + Fat (\%) showed a significant difference between tumor and fat, only in vivo, and between tumor and healthy colorectal wall, both in and ex vivo. Here again, changes between in vivo and ex vivo were in the same direction. For Fat/(Water + Fat) (\%), there was only a significant difference between tumor and fat, both in and ex vivo.

\subsubsection{Classification}

Eight different classifiers were created, using different train and test datasets. In four out of eight classifiers all parameters were used, for the other four classifiers 
only the selected parameters were used. In Figure 6.3 the ROC curves obtained from all four classifiers using all parameters are shown per tissue type. As can be seen, the classification trained on ex vivo data and tested on in vivo data performed similar to the other classifiers, which is supported by the AUC and MCC values (Table 6.6). Furthermore, McNemar's test showed that there was no significant difference between the results from the classifier trained and tested on in vivo data and the results from the classifier trained on ex vivo data and tested on in vivo data $(P=0.17)$.
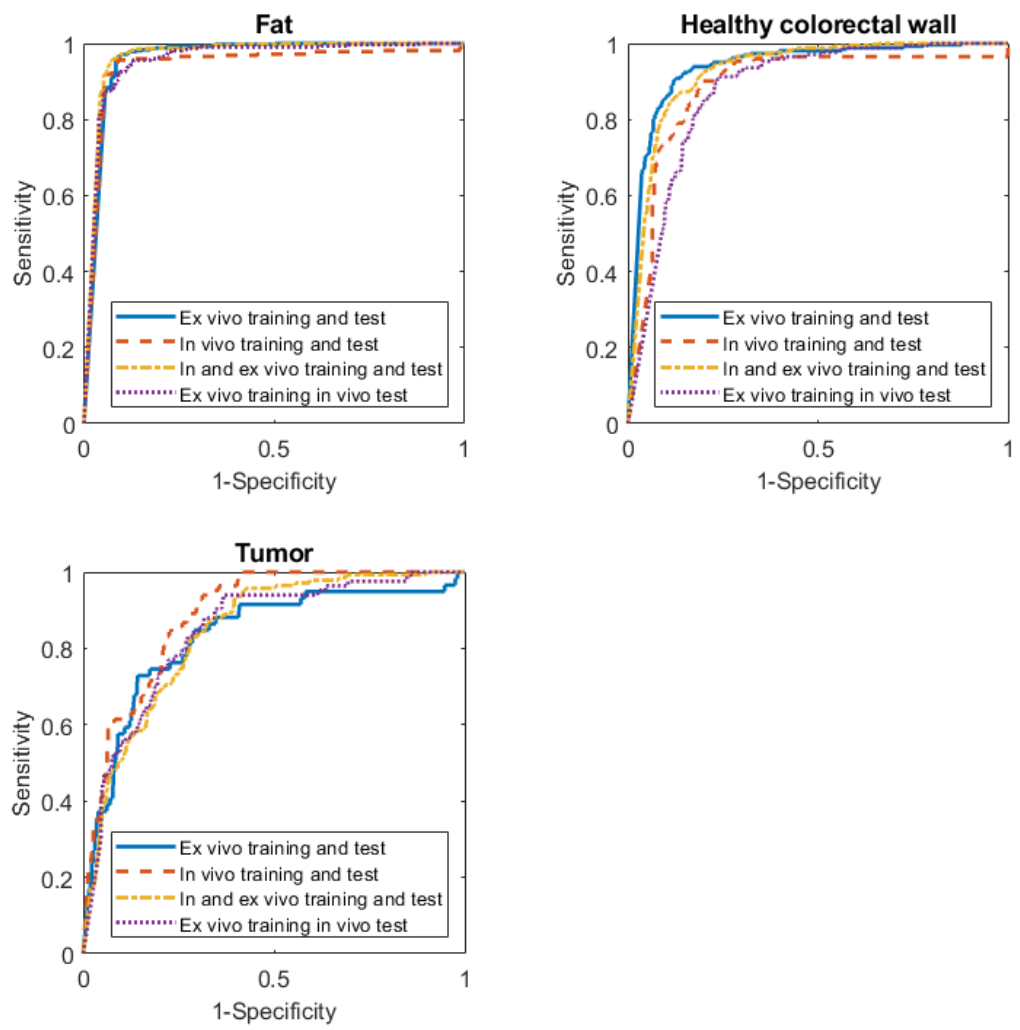

Figure 6.3: ROC curves per tissue type of classification using different train and test datasets.

In Figure 6.4, the ROC curves of the classifiers using the ex vivo data as train dataset and in vivo data as test dataset are shown for all parameters and for the selected parameters. The AUC of the ROC curve for fat remained the same when only the selected parameters were used (Table 6.6). For healthy colorectal wall 
Table 6.6: AUC and MCC values for all classifier results

\begin{tabular}{llllllll}
\hline & & \multicolumn{2}{c}{ Fat } & \multicolumn{2}{c}{ Healthy colorectal wall } & \multicolumn{2}{c}{ Tumor } \\
& & All & Selected & All & Selected & All & Selected \\
\hline \multirow{2}{*}{ Ex vivo training and test } & AUC & 0.96 & 0.95 & 0.94 & 0.92 & 0.83 & 0.84 \\
\multirow{2}{*}{ In vivo training and test } & MCC & 0.85 & 0.82 & 0.76 & 0.75 & 0.33 & 0.28 \\
& AUC & 0.94 & 0.96 & 0.89 & 0.92 & 0.89 & 0.87 \\
\multirow{2}{*}{ Ex and in vivo training and test } & MCC & 0.87 & 0.87 & 0.63 & 0.68 & 0.41 & 0.44 \\
\multirow{2}{*}{ Ex vivo training in vivo test } & AUC & 0.97 & 0.96 & 0.93 & 0.92 & 0.84 & 0.87 \\
& MCC & 0.87 & 0.86 & 0.73 & 0.74 & 0.33 & 0.40 \\
& AUC & 0.96 & 0.96 & 0.88 & 0.90 & 0.84 & 0.89 \\
& MCC & 0.82 & 0.86 & 0.62 & 0.68 & 0.48 & 0.46 \\
\hline
\end{tabular}

and tumor an increase in AUC was shown when only selected parameters were used, from 0.88 to 0.90 and from 0.84 to 0.89 respectively. The MCC values of fat and healthy colorectal wall increased when only selected parameters are used, whereas the MCC value of tumor decrease.

\subsection{Discussion}

In this study the potential of using ex vivo data to train a tissue classification for in vivo use is examined. AUC values of $0.96,0.88$ and 0.84 and MCC values of $0.82,0.62$ and 0.48 , for fat, healthy colorectal wall and tumor, respectively, for training on ex vivo data and testing on in vivo data were obtained. These values were comparable to the AUC and MCC values when training and testing was performed on in vivo data alone.

As a first step in this research a statistical analysis was done on the fitted parameters to examine if there was a significant difference between in vivo and ex vivo data. Most clear difference is seen for the Water + Fat (\%) and Fat/(Water + Fat) (\%) parameters. A significant decrease of Water + Fat (\%) was seen for all tissue types from in vivo to ex vivo. This most likely has to do with dehydration (vaporization and leakage) of the tissue when taken out of the patient. This is supported by the increase in Fat/(Water + Fat) (\%) parameter for all tissue types, showing a decrease in water content. Furthermore, for all tissues there was a significant difference in Blood (\%) and StO2 (\%) which were both increased when measured ex vivo compared to in vivo. Increase in Blood (\%) can be explained by an increase in blood volume in the capillaries after excision [19]. The increase in StO2 (\%) can be explained by the exposure to air and the decrease in oxygen consumption by the cells in the specimen. These results are not in agreement with results found 

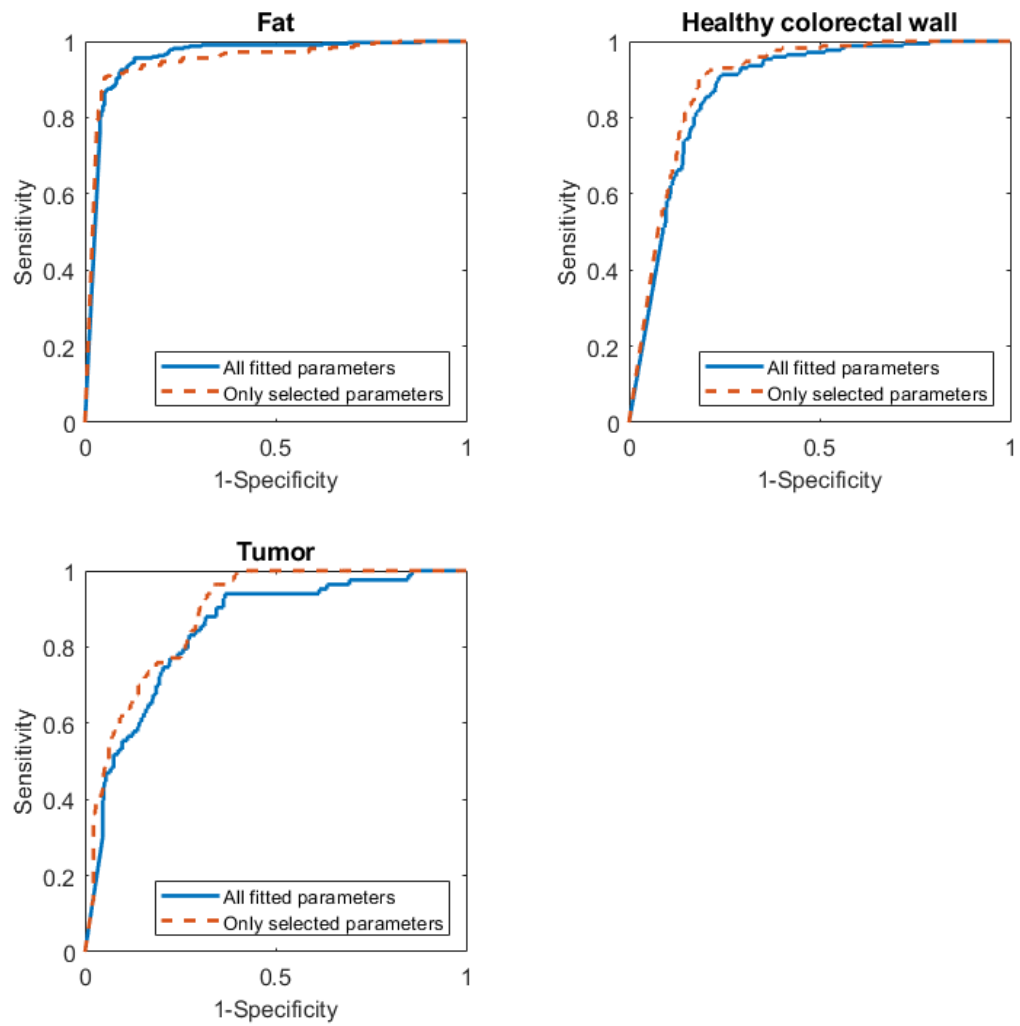

Figure 6.4: ROC curves per tissue type, classification trained with ex vivo data and tested on in vivo data. Two classifications are performed, one with all parameters and one with only the selected parameters.

by Salomatina et al. [18]. In their study comparing in and ex vivo measurements on mice ear, deoxygenation of the blood was found for the ex vivo measurements. The difference can be explained by the time between excision and measurement. In the study by Salomatina et al. the measurements were performed within 5-10 min after excision, while tissue may still be consuming oxygen, especially in the first few minutes. In our study the time between excision and measurement was up to one hour. This might increase the difference in oxygenation between in vivo and ex vivo in the current study. We measured the tissues within 1 hour after resection, based on the results from previous research results may differ when this time interval may be significantly longer. 
As stated by Jacques et.al., scattering parameters should be stable for a few hours after resection if over hydration and dehydration are avoided [23]. Most scattering parameters did indeed show no significant difference between in and ex vivo, except for scattering at $800 \mathrm{~nm}$ in fat, scattering slope in healthy colorectal wall and the fraction of Mie scattering versus Rayleigh scattering for tumor. As stated before, the decrease in Water + Fat (\%) and increase in Fat/(Water + Fat) (\%) most likely showed dehydration of the tissue. The changes in some scattering parameters between in vivo and ex vivo might be explained by this.

In the second step, an SVM was trained and tested on four different combinations of train and test data. When ex vivo data was used for training and in vivo data was used for testing similar results were obtained compared to the other classifiers. This showed that using ex vivo data to create a classifier and using it afterwards to classify in vivo data will give similar results compared to a complete in vivo study in which both training and testing of the classifier is performed on in vivo data. Using McNemar's test, no significant difference between the results of these two classifiers was found $(p=0.17)$.

The classification results were similar if only selected parameters were used for classification. The parameters that were selected for the final classification were selected based on the results of three forward feature selections. The selected parameters did show changes between the in vivo and ex vivo setting. Parameters that are significantly different between in vivo and ex vivo might not seem useful in the classification at first. However, if these parameters either increase or decrease systematically for all tissue types, differences between tissues may still be present and they might still be of added value to the classification. For the parameters that were not selected, any differences between tumor and fat and between tumor and healthy colorectal wall that were present ex vivo, were absent or at least different for the in vivo setting.

For this study an analytical model based on the diffusion theory was used to extract different absorption and scattering properties from the reflectance spectra of the tissue. The resulting parameters of the fit of the analytical model were used for classification instead of intensity values of the spectra as was done previously [12].The fitted parameters were used because changes in these parameters between in vivo and ex vivo could be related to biological parameters and processes. Furthermore, because the model takes into account the fiber arrangement, results are not dependent on the measurement set-up and the conclusions are therefore applicable in a more general sense. However, care should be taken 
that the assumptions made in the fit model are appropriate. One of the assumptions made in the diffusion theory is that the tissue is homogeneous. One can doubt whether this is an appropriate assumption for the colorectal wall which consists of several layers. However, this problem is present in the in vivo setting as well as in the ex vivo setting. Moreover, bad performance of the model due to for instance layered tissue will result in bad fits, which were excluded from the analysis. In this study $11 \%$ (187 of the 1605 measurements) of the measurements were excluded based on bad performance of the fit model. So, by using the analytical model, and excluding measurements with a bad performing fit, the results obtained in this study are expected to be generalizable to other measurement set-ups.

\subsection{Conclusion}

Based on the results obtained in this study, it can be concluded that at least for colorectal cancer it is possible to train a classifier intended to classify in vivo spectra reliably using ex vivo measurements. Only parameters, that are constant between in vivo and ex vivo or change similarly over tissue types, should be used for that classification. Because ex vivo data acquisition is simpler compared to in vivo, larger databases can be used for training of a classifier that can be used in vivo. This might accelerate the development of optical techniques for surgical applications. 


\section{References}

[1] F. Bray et al., "Global cancer statistics 2018: GLOBOCAN estimates of incidence and mortality worldwide for 36 cancers in 185 countries," CA: A Cancer Journal for Clinicians, vol. 68, pp. 394424, nov 2018.

[2] J. S. Park et al., "A Circumferential Resection Margin of $1 \mathrm{~mm}$ Is a Negative Prognostic Factor in Rectal Cancer Patients With and Without Neoadjuvant Chemoradiotherapy," Diseases of the Colon \& Rectum, vol. 57, no. 8, 2014.

[3] I. D. Nagtegaal and P. Quirke, "What Is the Role for the Circumferential Margin in the Modern Treatment of Rectal Cancer?," Journal of Clinical Oncology, vol. 26, no. 2, pp. 303-312, 2008.

[4] T. J. Farrell, M. S. Patterson, and B. Wilson, "A diffusion theory model of spatially resolved, steady-state diffuse reflectance for the noninvasive determination of tissue optical properties in vivo," Medical Physics, vol. 19, no. 4, pp. 879-888, 1992.

[5] T. M. Bydlon et al., "Chromophore based analyses of steady-state diffuse reflectance spectroscopy: current status and perspectives for clinical adoption," Journal of Biophotonics, vol. 8, pp. 9-24, jan 2015.

[6] D. J. Evers et al., "Diffuse reflectance spectroscopy: toward real-time quantification of steatosis in liver," Transplant International, vol. 28, no. 4, pp. 465-474, 2015.

[7] L. L. Boer et al., "Fat/water ratios measured with diffuse reflectance spectroscopy to detect breast tumor boundaries," Breast Cancer Research and Treatment, vol. 152, no. 3, pp. 509-518, 2015.

[8] J. W. Spliethoff et al., "Improved identification of peripheral lung tumors by using diffuse reflectance and fluorescence spectroscopy," Lung Cancer, vol. 80, no. 2, pp. 165-171, 2013.

[9] G. C. Langhout et al., "Differentiation of healthy and malignant tissue in colon cancer patients using optical spectroscopy: A tool for image-guided surgery," Lasers in Surgery and Medicine, vol. 47, no. 7, pp. 559-565, 2015.

[10] R. M. Schols, P. Dunias, F. P. Wieringa, and L. P. S. Stassen, "Multispectral characterization of tissues encountered during laparoscopic colorectal surgery," Medical Engineering and Physics, vol. 35, no. 7, pp. 1044-1050, 2013.

[11] S. G. Brouwer de Koning et al., "Toward complete oral cavity cancer resection using a handheld diffuse reflectance spectroscopy probe," Journal of Biomedical Optics, vol. 23, pp. 1-9, oct 2018.

[12] E. J. M. Baltussen et al., "Diffuse reflectance spectroscopy as a tool for real-time tissue assessment during colorectal cancer surgery," Journal of biomedical optics, vol. 22, no. 10, pp. 106014106016, 2017.

[13] G. Zonios et al., "Diffuse reflectance spectroscopy of human adenomatous colon polyps in vivo," Applied optics, vol. 38, no. 31, pp. 6628-6637, 1999. 
[14] H.-W. Wang et al., "Diffuse reflectance spectroscopy detects increased hemoglobin concentration and decreased oxygenation during colon carcinogenesis from normal to malignant tumors.," Optics express, vol. 17, no. 4, pp. 2805-17, 2009.

[15] H. K. Roy et al., "Spectroscopic Microvascular Blood Detection From the Endoscopically Normal Colonic Mucosa: Biomarker for Neoplasia Risk," Gastroenterology, vol. 135, no. 4, pp. 10691078, 2008.

[16] A. Dhar et al., "Elastic scattering spectroscopy for the diagnosis of colonic lesions: initial results of a novel optical biopsy technique," Gastrointestinal Endoscopy, vol. 63, pp. 257-261, feb 2006.

[17] J. R Mourant et al., Elastic scattering spectroscopy as a diagnostic tool for differentiating pathologies in the gastrointestinal tract: Preliminary testing, vol. 1. apr 1996.

[18] E. Salomatina and A. N. Yaroslavsky, "Evaluation of thein vivoandex vivooptical properties in a mouse ear model," Physics in Medicine and Biology, vol. 53, no. 11, pp. 2797-2807, 2008.

[19] G. C. Langhout et al., "Nerve detection using optical spectroscopy, an evaluation in four different models: In human and swine, in-vivo, and post mortem," Lasers in surgery and medicine, vol. 50, no. 3, pp. 253-261, 2018.

[20] R. Nachabe et al., "Estimation of lipid and water concentrations in scattering media with diffuse optical spectroscopy from 900 to 1600 nm," vol. 15, pp. 37010-37015, 2010.

[21] R. Nachabé et al., "Estimation of biological chromophores using diffuse optical spectroscopy: benefit of extending the UV-VIS wavelength range to include 1000 to $1600 \mathrm{~nm}$," Biomedical Optics Express, vol. 1, no. 5, pp. 1432-1442, 2010.

[22] T. G. Dietterich, "Approximate Statistical Tests for Comparing Supervised Classification Learning Algorithms," Neural Computation, vol. 10, pp. 1895-1923, oct 1998.

[23] S. L. Jacques, "Optical properties of biological tissues: a review," Physics in Medicine \& Biology, vol. 58, no. 11, p. R37, 2013. 


\section{Chapter 7}

\section{Optimizing tissue classification algorithms in colorectal cancer using diffuse reflectance}

Elisabeth J.M. Baltussen, Henricus J.C.M. Sterenborg, Theo J.M. Ruers, Behdad Dashtbozorg, Biomedical Optics Express, 2019, Volume 10 (12) 


\subsection{Abstract}

Diffuse reflectance spectroscopy can be used in colorectal cancer surgery for tissue classification. The main challenge in the classification task is to separate healthy colorectal wall from tumor tissue. In this study, four normalization techniques, four feature extraction methods and five classifiers are applied to nine datasets, to obtain the optimal method to separate spectra measured on healthy colorectal wall from spectra measured on tumor tissue. All results are compared to the use of the entire non-normalized spectra. It is found that the most optimal classification approach is to apply a feature extraction method on non-normalized spectra combined with support vector machine or neural network classifier. 


\subsection{Introduction}

In both men and women, colorectal cancer is the third most common cancer worldwide [1]. The standard care for patients with colorectal cancer is surgery [2]. During colorectal cancer surgery, the surgeon must rely on visual and tactile feedback to recognize the tumor boundaries. As a result, there are two main issues: complete removal of the tumor and preventing complication due to too extensive surgery. To obtain an optimal balance between these two issues, intra-operative tissue recognition would be of great benefit to provide the surgeon with information about the tissue he or she encounters.

Diffuse reflectance spectroscopy (DRS) is an optical technique that has been used for tissue recognition in cancer diagnostics [3-10]. Light over a broad wavelength range is sent into the tissue, where the light interacts by scattering and absorption. Part of this light will be scattered back to the surface of the tissue, where it can be detected. In fiberoptic DRS, light is transported through fibers, both from source to tissue and from the tissue surface to the spectrometers [11]. In the fiberoptic geometry only point measurements can be performed. Using hyperspectral imaging $(\mathrm{HSI})$, the tissue samples are directly illuminated by a broadband light source and the reflected light over the entire illuminated surface is collected by a camera. Therefore, a complete specimen can be imaged at once. With HSI a 3D data-cube is collected, in which two dimensions contain the spatial information about the tissue sample and the third dimension represents the wavelength information for each spatial pixel [10].

Both fiberoptic DRS and diffuse reflection HSI have been used in tissue classification in colorectal cancer before. For HSI the main applications in colorectal cancer so far were on imaging of the pathology slides for automatic pathology classification [12-16] and on colonoscopy in which tumor was distinguished from healthy colorectal mucosa, imaged from within the lumen [17-19]. The work presented in the current paper aims to detect cancer during colorectal surgery. During surgery tumor is approached from outside the lumen. To best of our knowledge, there has only been one study focused on the use of HSI in colorectal cancer surgery in which the tumor is assessed from outside of the lumen and should be distinguished from fat and healthy colorectal wall [20]. In this work performed by our group tissue samples from colorectal cancer patients were obtained after resection and imaged by two hyperspectral cameras operating in the visual (400-1000 $\mathrm{nm})$ and near-infrared $(900-1700 \mathrm{~nm})$ wavelength range. The mean accuracy to 
distinguish fat, healthy colorectal wall and tumor was 0.88 [20].

For fiberoptic DRS the main focus in colorectal cancer has also been on the application in colonoscopy for assessing the mucosa from within the colorectal lumen [21-25]. On the topic of application during colorectal cancer surgery a few papers have been published so far. In an ex vivo study by Schols et al. tumor could be discriminated from healthy surrounding tissue [7]. In an ex vivo study performed by our own group the accuracy to distinguish tumor tissue from fat and healthy colorectal wall was 0.95 [26].

An important step in developing a diagnostic algorithm based on spectral information is feature extraction, reducing the data used for classification, focusing on that part of the data that contains the crucial information. In previous studies, many different feature extraction and classification techniques have been used to analyze the spectra obtained with fiberoptic DRS or diffuse reflection HSI. In fiberoptic DRS for instance a fit algorithm based on photon diffusion theory has been used to extract absorption and scattering parameters from the measured spectra and to translate these into a limited set of parameters describing tissue composition and scattering behavior [27]. Based on these parameters tissue types could be distinguished $[4,5,11,28]$. Schols et al. used several amplitudes and gradients for classification [29]. In our group, spectral bands were used for both HSI and DRS data to reduce the number of features $[20,26]$. Classification of tissue types has been done in several ways. First of all, direct comparison of single features or simple combinations of features, has been performed to distinguish different tissues [4, 30-34]. In addition, several machine learning techniques have been used for the tissue classification such as classification and regression tree algorithms [5, 6, 35-37], linear discriminant analysis (LDA) [24, 38-40] and support vector machines (SVM) [3, 26, 20, 37, 38, 41-43].

Previous studies used different analysis techniques to classify tissue during colorectal cancer surgery $[6,26,20]$. The current study will systematically examine several options to optimize the classification of healthy colorectal wall versus tumor tissue in colorectal cancer. Several different normalization and feature extraction techniques will be used in combination with a variety of classifiers to determine the optimal classification strategy. 


\subsection{Materials and methods}

\subsubsection{Data}

In this study, datasets included were obtained using two different colorectal tissue sample sets. The measurements performed on tissue sample set 1 were only done ex vivo and were all performed using the same fiberoptic probe, which includes four fiber distances. The measurements performed on tissue sample set 2 were done both in vivo and ex vivo and were performed with disposable probes, so for each new patient a different probe with identical design was used. When measurements were performed using fiberoptic DRS, data of two fiber distances was acquired at the same time. There was only one diffuse reflection HSI dataset included which was obtained on tissue sample set 1 (Figure 7.1). This dataset was a combination of data obtained with a visual hyperspectral camera and data obtained with a near-infrared hyperspectral camera. In total nine datasets were included for the current analysis (Figure 7.1). These datasets are separated based on the sample sets, ex vivo or in vivo, fiberoptic DRS or diffuse reflection HSI and for fiberoptic DRS the fiber distance used. To avoid confusion, in the rest of the paper the datasets will be referred to by the assigned number as shown in Figure 7.1.

In Table 7.1 an overview is given on the number of patients and the number of spectra present in all datasets, for dataset $\# 5$ the number of spectra is equal to the number of pixels. Classification in the current study was only performed on colon and tumor tissue because previous studies have shown that discriminating fat from other tissue types is an easy and already solved task [20, 26]. For example, using fiberoptic DRS measurements on ex vivo samples (dataset \#4) an accuracy of $1.00 \pm 0.00$ was obtained for the classification of fat [26]. When using diffuse reflection $\mathrm{HSI}$ (dataset \#5) the same accuracy of 1.00 was achieved for fat classification [20]. Based on these results it was decided not to include fat measurements into the current analysis and to focus on the more challenging task of tumor/colon classification.

\subsubsection{Preprocessing}

Before analysing the spectra, all spectra were calibrated using a white reference, obtained on a Spectralon sample, and a dark reference. In the spectra obtained, intensity differences might have been present that were not due to tissue specific 


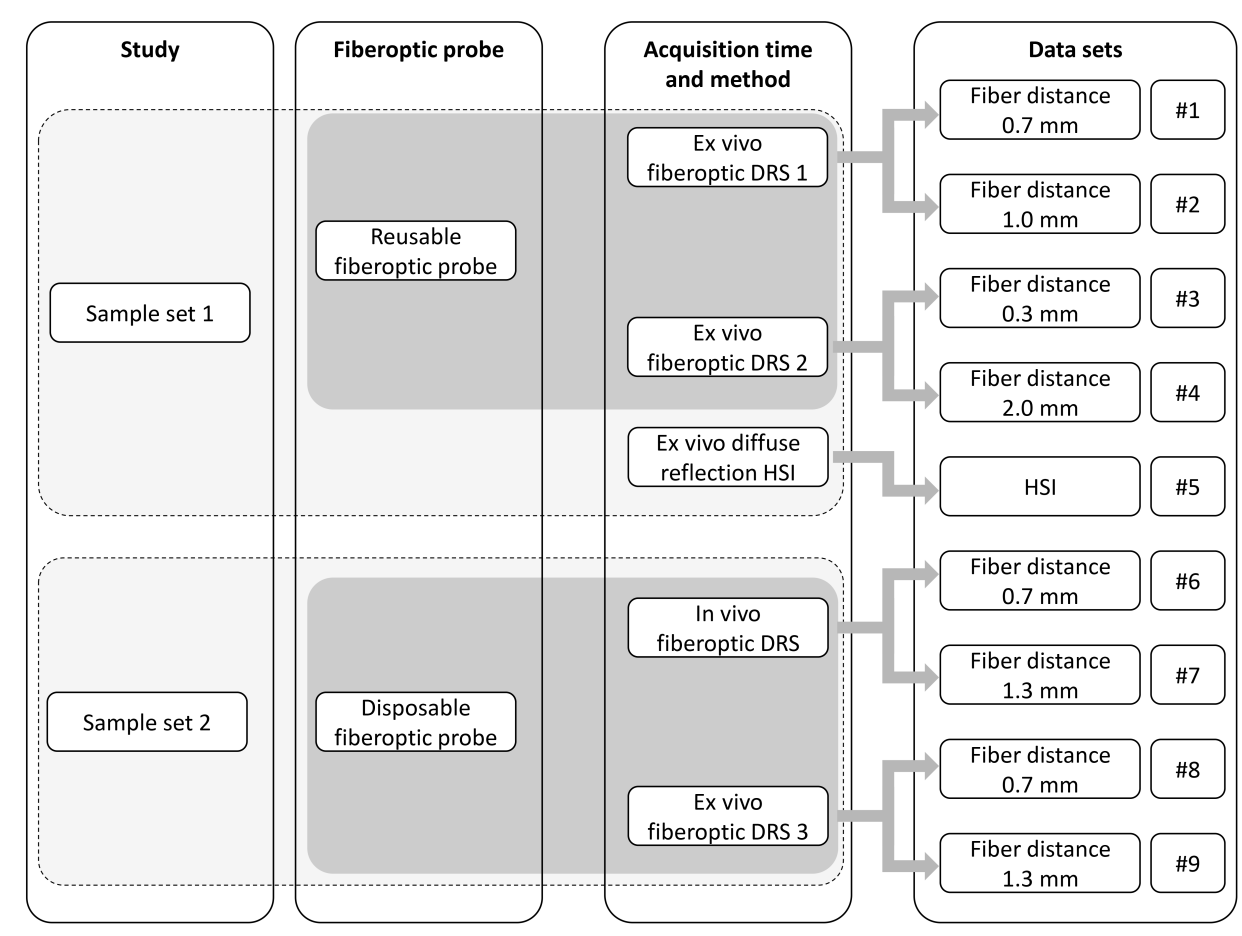

Figure 7.1: Overview of all datasets used in this study, the first column shows the different sample sets, the second column what type of fiberoptic probe was used (reusable or disposable), the third column the difference in acquisition time (ex vivo/in vivo) and method (DRS/HSI) and the fourth shows different fiber distances and all datasets with assigned numbers. The light and dark gray areas indicate datasets obtained on the same sample set and datasets obtained with the same DRS probe type respectively.

difference but due to for instance difference in the distance between the illumination source and the sample, compared to the distance between the illumination source and the Spectralon sample used for calibration. Calibration is performed to standardize all measurements. However, to standardize, the distance between the illumination source and the measured sample should be the same as the distance between the illumination source and the Spectralon sample. When the tissue thickness deviates over the sample, the distance between the illumination source and tissue will not be similar for the entire sample, causing intensity differences. Moreover, the coupling between the fibers and spectrometer might cause a variation in detection efficiency of the signal. To compensate for these intensity differences, spectra can be normalized before classification or feature extraction. In this study, three different normalization techniques were compared to each other and to no normalization. First, spectra were normalized by their intensity at 
Table 7.1: Overview of number of patients and number of spectra per dataset and per tissue type.

\begin{tabular}{|c|c|c|c|c|}
\hline \multirow{2}{*}{ Dataset } & \multicolumn{2}{|c|}{ Number of patients } & \multicolumn{2}{|c|}{ Number of spectra } \\
\hline & Over all & Per tissue type & Over all & Per tissue type (\%) \\
\hline$\# 1$ and \#2 & 38 & $\begin{array}{l}\text { Colon: } 32 \\
\text { Tumor: } 29\end{array}$ & 948 & $\begin{array}{l}\text { Colon: } 456 \text { (48.1) } \\
\text { Tumor: } 492(51.9)\end{array}$ \\
\hline \#3 and \#4 & 38 & $\begin{array}{l}\text { Colon: } 32 \\
\text { Tumor: } 29\end{array}$ & 943 & $\begin{array}{l}\text { Colon: } 450 \text { (47.7) } \\
\text { Tumor: } 493 \text { (52.3) }\end{array}$ \\
\hline \#5 & 29 & $\begin{array}{l}\text { Colon: } 22 \\
\text { Tumor: } 27\end{array}$ & 1328 & $\begin{array}{l}\text { Colon: } 569 \text { (42.8) } \\
\text { Tumor: } 759 \text { (57.2) }\end{array}$ \\
\hline$\# 6$ and \#7 & 28 & $\begin{array}{l}\text { Colon: } 28 \\
\text { Tumor: } 14\end{array}$ & 364 & $\begin{array}{l}\text { Colon: } 261 \text { (71.7) } \\
\text { Tumor: } 103 \text { (28.3) }\end{array}$ \\
\hline$\# 8$ and \#9 & 28 & $\begin{array}{l}\text { Colon: } 28 \\
\text { Tumor: } 12\end{array}$ & 398 & $\begin{array}{l}\text { Colon: } 321(80.7) \\
\text { Tumor: } 77(19.3)\end{array}$ \\
\hline
\end{tabular}

$800 \mathrm{~nm}$ [32]. This wavelength was chosen because no absorption was assumed to be present at this wavelength. After normalization the intensity of all reflectance spectra should be equal to 1 at $800 \mathrm{~nm}$. As a second option, the spectra were normalized using the area under the curve (AUC), setting all AUCs to 1. Finally, a standard normal variate (SNV) normalization was used [44, 45]. Using SNV normalization, the mean of each spectrum was set to zero and the standard deviation was set to one. The SNV is often used for diffuse reflection HSI to exclude the influence of glare on the spectra [46, 47]. However, SNV normalization also removes information on the scattering. As shown in Figure 7.2, for the fiberoptic DRS and diffuse reflection HSI datasets all 4 different normalization techniques were evaluated.

\subsubsection{Feature extraction}

When the number of features is very large relative to the number of samples in a dataset, some classifiers struggle to train effective models, which is called curse of dimensionality [48]. This is especially relevant for algorithms that rely on distance calculations like kNN [49]. Feature extraction and features selection techniques can be used to avoid the curse of dimensionality. Feature extraction creates a new, smaller set of features that still captures most of the useful information.

Besides using the entire spectra (1151 features) for classification, four feature extraction methods were evaluated. First, the seven most prominent peaks of a spectrum were selected, from these peaks the intensity values, the locations 


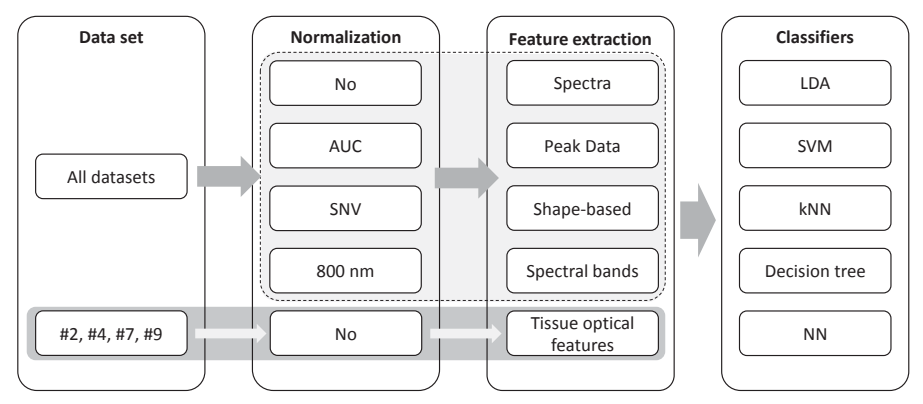

Figure 7.2: Overview of normalization, feature extraction and classification methods per dataset.

and the right side gradient of the peaks were used as features (Figure 7.3a). In the second feature extraction technique, 23 features were selected manually to describe the coarse shape of the spectra while emphasizing the differences between different tissue classes. Features included were: the locations of maxima (4), locations of minima (2), gradients (9), prominence values (4), widths (2) and intensity value (1) (Figure 7.3b). The prominence values were defined as the intrinsic height of a peak relative to neighboring dips. As a third feature set, spectral bands were selected using k-means clustering (Figure 7.3c) [20, 26]. The spectra were divided into at least 10 bands based upon the intensity values. Random start points were set after which the spectra were divided into 10 groups using k-means clustering based on the intensity values. If wavelengths within one group were not continuous, the group was divided into subgroups with only continuous wavelengths. In the final feature extraction method, tissue constituents were determined per spectrum using a fit algorithm, based on photon diffusion theory (Figure $7.3 d$ ) $[11,50,51]$. With this algorithm, reflectance spectra of selected chromophores were fitted to the measured reflectance spectra to obtain the composition of the measured tissue in a number of parameters [27]. For these tissue types, 12 parameters were used for the fit, which were used as features for the classification. The fit algorithm is only valid for the fiberoptic geometry, and only the measurements in which the fiber distance between the receiving and collecting fiber was at least $1 \mathrm{~mm}$ [50]. Therefore, features based on fit results were only extracted for datasets \#2, \#4, \#7 and \#9, which are all fiberoptic DRS datasets with fiber distances of at least $1 \mathrm{~mm}$. No normalization was used in case of tissue optical feature extraction, because the analytical model is based on the 


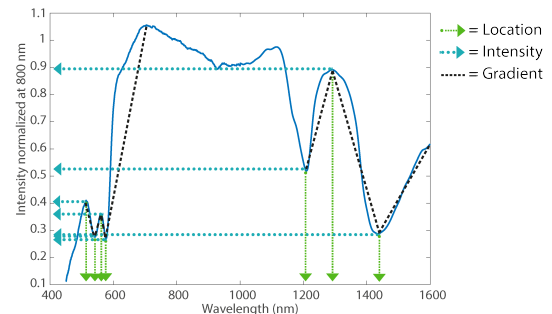

(a)

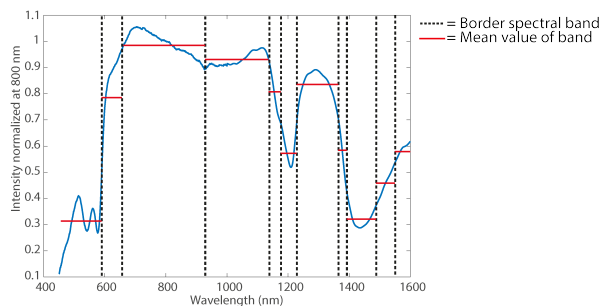

(c)

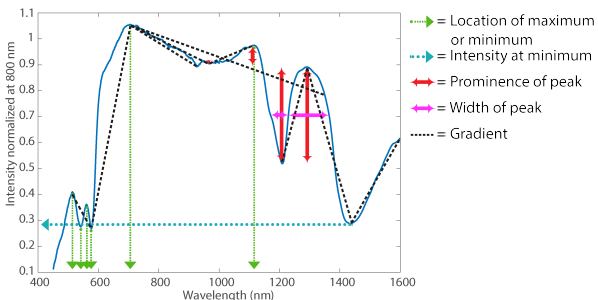

(b)

\begin{tabular}{|l|l|}
\hline Parameter: & Value: \\
\hline Blood (\%) & 1.62 \\
\hline $\mathrm{StO}_{2}(\%)$ & 72.73 \\
\hline Water + Fat (\%) & 82.76 \\
\hline Fat/Water+Fat (\%) & 65.31 \\
\hline Scattering at $800 \mathrm{~nm}\left(\mathrm{~cm}^{-1}\right)$ & 13.99 \\
\hline Scattering slope (-) & 0.76 \\
\hline Scale factor (-) & 0.11 \\
\hline Blood vessel diameters $(\mu \mathrm{m})$ & 0.20 \\
\hline Mie vs. Rayleigh scattering $(-)$ & 1.00 \\
\hline Collagen (\%) & 5.00 \\
\hline Beta-carotene $(\mathrm{m} \mu \mathrm{M})$ & 4.97 \\
\hline Methemoglobin $(\%)$ & 1.00 \\
\hline
\end{tabular}

(d)

Figure 7.3: Four different feature extraction methods shown for a spectrum obtained on fat tissue. (a) Wavelengths, intensities and gradients of seven most prominent peaks. (b) Wavelengths, intensities, prominences, widths and gradients describing the shape of the entire spectrum. (c) Spectral bands, the red bars indicate the mean intensity value per band. (d) Fitted parameters using the analytical reflectance model.

reflectance signal, which should not be normalized. In Figure 7.2 an overview is given on which datasets are subjected to all possible combinations of feature extraction and normalization techniques.

\subsubsection{Classifiers}

In machine learning, the "No Free Lunch" theorem states that there is no one model that works best for every problem [52]. The assumptions of a great model for one problem may not hold for another problem, so it is common in machine learning to try multiple classifiers and choose the one that works best for that particular application. In this study five different supervised classification algorithms were used to distinguish colon from tumor, k-nearest neighbor $(k N N)$, a decision tree classier, LDA, SVM, and a neural network (NN). All classifiers were trained and tested using Matlab 2018a (MathWorks Inc., Natick, Massachusetts, US). For the $k N N$ classifier, five neighbors were used to determine the class. The decision tree was a binary decision tree with "Gini's diversity index" as a split criterion and 
the maximal number of branch node splits and minimum parent size were set 3 and 10 , respectively. For the SVM a linear SVM was selected. For the NN classifier, a feedforward network was used with a single layer of 20 hidden neurons and a scaled conjugate gradient backpropagation training function. For all classifiers, the features were standardized to a zero mean and standard deviation of one per parameter before used in the classification. Standardization was determined based on the data from the train dataset and thereafter applied to the test dataset.

\subsubsection{Data analysis}

The sample sets were partitioned once into a train and test set, $80 \%$ of the patients was used to train and $20 \%$ to test the classifiers. The proportion of healthy versus tumor measurements is the same in the train and test dataset. The classifiers were evaluated using the accuracy, the area under the ROC curve (AUC) and the Matthews correlation coefficient (MCC). The accuracy and AUC are commonly used performance measures. However, these performance metrics do not take into account unbalanced classes in each dataset. Therefore, the MCC, which is less sensitive to unbalanced datasets, was added as a performance measure [53]. The MCC ranges from -1 to 1 , with 0 indicating a classification no better than random, -1 total disagreement in the classification and +1 a perfectly correct classification.

In supervised learning, overfitting happens when classifiers are trained on the noise along with the underlying pattern in the data. It happens when the classifier is trained on noisy or unrepresentative train data. Complex models like decision trees are more prone to overfitting. Overfitting can be recognized by examining the classifier variance, which is the variability of the model prediction for given data samples. Classifiers with high variance perform well on the train dataset but are not able to generalize to data it has not seen before e.g. the test dataset. Therefore, a large drop from training accuracy to test accuracy will be present in overfitting.

The classifications, done with all classifiers, on all different combinations shown in Figure 7.2, were first tested for overfitting of the classifiers. This was done by subtracting the test accuracy from the training accuracy for each combination of normalization, feature extraction and classifier for each dataset. Since there is no general rule to define overfitting, we set the threshold for overfitting by a decrease in accuracy from train to test of 0.15 . If the decrease in accuracy was above 0.15 , 
the classifier was defined to be overfitting during training.

Thereafter, the three normalization techniques were compared to the use of no normalization prior to classification. The accuracy and MCC values of the nonnormalized data were considered as a baseline and were subtracted from the accuracy and MCC values obtained on the normalized data. In this analysis feature extraction methods were not included, so only entire spectra were used. The results were averaged per normalization technique and per dataset to assess if the normalization might have a different influence on different datasets.

A similar analysis was done for the feature extraction methods. These were all compared to the use of the entire spectrum during classification (baseline performance). Again, the difference in MCC and accuracy values compared to baseline were determined, for the selected classifiers and all datasets. However, this time only the selected normalization technique from the previous analysis was used to limit the amount of analyses. A mean difference in MCC and accuracy was obtained per feature extraction method and also the influence of feature extraction techniques for each dataset was obtained.

The combination of normalization and feature extraction was also examined using the difference in accuracy and MCC. The combination of all feature extraction methods and all normalization methods were compared to no normalization and the use of the entire spectrum.

Finally, for each dataset the combination of normalization, feature extraction and classifier with the maximum MCC and accuracy values were extracted from the data. These results were compared to the MCC and accuracy values of each dataset, obtained using the overall most optimal combination of normalization, feature extraction and classifier, as determined by all previous analysis.

\subsection{Results}

\subsubsection{Overfitting}

To evaluate the classifiers overfitting, the test accuracy of each classifier was subtracted from the train accuracy. A maximum decrease in accuracy of 0.15 from train to test was chosen as the threshold for overfitting. In the histograms of Figure 7.4, the number of times (y-axis) a certain difference between train and test accuracy (x-axis) was obtained, is shown. The histograms in Figure 7.4 are colored per classifier. In Figure $7.4 \mathrm{a}$, the results using no normalization and no 
feature extraction method are shown. Here it clearly shows that the chances of overfitting were largely classifier dependent. For instance, for the NN classifier (green) most results were obtained below the overfitting threshold, the same accounts for SVM. Whereas for the decision tree (orange), all results of train minus test accuracy were above the threshold, indicating overfitting of the classifier, the same accounts for $k N N$ and LDA. In Figure 7.4b normalized data shown, this shows that there was no influence of the normalization on the overfitting of the classifiers, because the distributions for all classifiers were similar to Figure 7.4a. Figure 7.4c includes all feature extraction methods, but only on non-normalized data. As shown, feature extraction did show improvement in the overfitting for the $k N N$ and LDA classifiers, as both distributions moved to the left of the graph (Figure 7.4c) compared to Figure 7.4a. With the use of feature extraction, more train minus test accuracies stayed below the threshold of overfitting. Overall, SVM and NN classifiers showed the least overfitting on all datasets. Therefore, further analysis was focused only on SVM and NN.

\subsubsection{Normalization}

The added value of normalization on the classification outcome was examined. To this end, the increase in accuracy and increase in MCC for each normalization compared to no normalization were analyzed. This analysis was performed for the entire spectrum and only using the SVM and NN classifiers as selected in the previous section. The average increase in MCC and accuracy values per normalization technique are shown in Table 7.2. In Table 7.2 it shows that there was no normalization technique that improved the outcome significantly for all datasets using the SVM or NN classifier. Similar results were obtained for the $k N N$, decision tree and LDA classifiers, which are not included in the Table 7.2.

Because of the large standard deviations, the increase in MCC and accuracy was evaluated per dataset as well. An increase was seen for all datasets which were obtained using the disposable fiberoptic DRS probes (datasets \#6 to \#9) using SNV and normalization at $800 \mathrm{~nm}$ technique, whereas mainly a decrease was seen for the datasets obtained with the reusable fiberoptic DRS probe (datasets \#1 to \#4) (Table 7.3). Normalization using AUC shows a decrease in MCC and accuracy for datasets \#8 and \#9 as well. For the dataset of the diffuse reflection HSI (dataset \#5) normalization did not seem to affect the results. Because, SNV normalization and normalization at $800 \mathrm{~nm}$ seemed only useful for some 


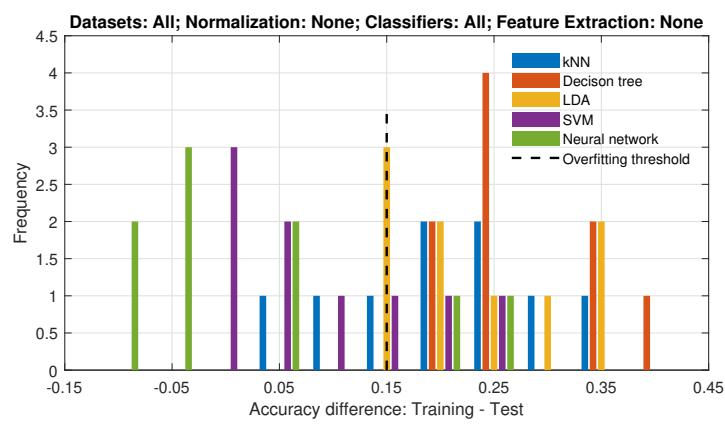

(a)

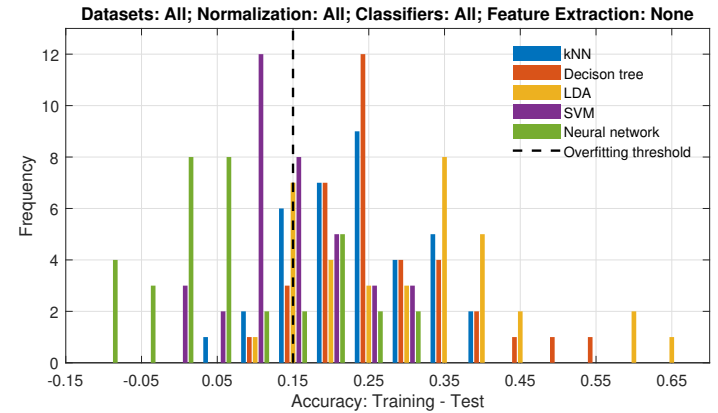

(b)

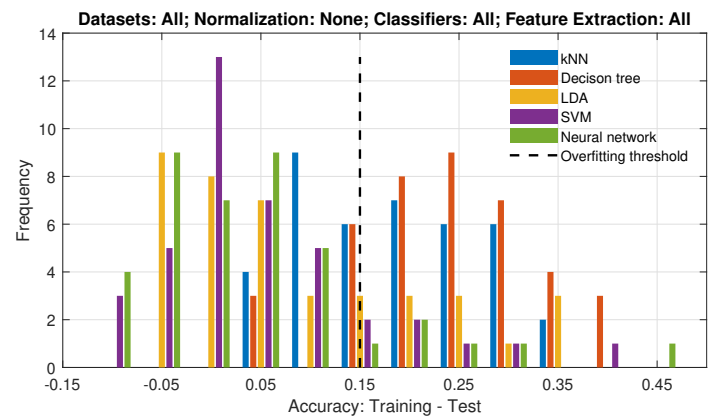

(c)

Figure 7.4: Histogram of the difference in accuracy between training and test and the number of times this difference is obtained. All classifiers are visualized separately. (a) Data is shown for all datasets, using no normalization and no feature extraction. (b) Data is shown for all datasets, using all normalizations and no feature extraction. (c) Data is shown for all datasets, using no normalization and all feature extractions.

datasets and AUC normalization did not seem useful at all, it was decided to continue without using normalization for the comparison of different feature extraction technique in the next section. 
Table 7.2: Mean increase in MCC and accuracy for all normalization techniques compared to no normalization for SVM and NN classifiers.

\begin{tabular}{lcc}
\hline Normalization & Increase in MCC (mean \pm std) & Increase in ACC (mean \pm std) \\
\hline SNV & $0.05 \pm 0.20$ & $-0.01 \pm 0.07$ \\
AUC & $0.02 \pm 0.17$ & $-0.01 \pm 0.07$ \\
Normalization at $800 \mathrm{~nm}$ & $0.02 \pm 0.15$ & $-0.01 \pm 0.08$ \\
\hline
\end{tabular}

$\mathrm{MCC}=$ Matthews Correlation Coefficient, $\mathrm{ACC}=$ Accuracy.

Table 7.3: Mean change per dataset in MCC value and accuracy, when normalization technique is compared to no normalization. Values of the standard deviation are not shown in the table, but none of the change values differed significantly from zero.

\begin{tabular}{|c|c|c|c|c|c|c|}
\hline & \multicolumn{2}{|c|}{ SNV normalization } & \multicolumn{2}{|c|}{ AUC normalization } & \multicolumn{2}{|c|}{ normalization at $800 \mathrm{~nm}$} \\
\hline & MCC & ACC & MCC & ACC & MCC & ACC \\
\hline \#1 & -0.04 & -0.02 & 0.01 & 0.00 & -0.03 & -0.01 \\
\hline \#2 & 0.04 & 0.02 & -0.02 & -0.01 & -0.03 & -0.01 \\
\hline \#3 & -0.17 & -0.08 & -0.09 & -0.04 & -0.06 & -0.03 \\
\hline \#4 & -0.03 & -0.01 & -0.06 & -0.03 & 0.01 & 0.00 \\
\hline \#5 & -0.04 & -0.02 & 0.00 & 0.00 & 0.00 & 0.00 \\
\hline \#6 & 0.14 & -0.04 & 0.11 & -0.03 & 0.07 & 0.06 \\
\hline \#7 & 0.20 & 0.03 & 0.30 & 0.09 & 0.17 & 0.07 \\
\hline \#8 & 0.08 & -0.05 & -0.04 & -0.01 & 0.03 & -0.07 \\
\hline$\# 9$ & 0.22 & 0.04 & -0.07 & -0.04 & 0.01 & -0.04 \\
\hline
\end{tabular}

MCC $=$ Matthews Correlation Coefficient, ACC $=$ Accuracy.

\subsubsection{Feature extraction}

Based on the results from the normalization, the first analysis on feature extraction was performed on non-normalized data. In Table 7.4 the results are shown for the mean increase in MCC and accuracy for all feature extraction methods compared to no feature extraction, e.g. the entire spectrum. Here, all datasets were used, no normalization was used and only the results from the SVM and NN classifiers were taken into account. From Table 7.4, it can be concluded that overall using tissue optical features improved classification results compared to the use of the entire spectrum. It should be noted that tissue optical features were only available for datasets for the fiber optic DRS data with fiber distance more than $1 \mathrm{~mm}$ (e.g. datasets \#2, \#4, \#7 and \#9). For the shape-based features and spectral bands almost no difference compared to using the entire spectrum for classification was seen. Peak data showed a drop in both MCC and accuracy compared to using the entire spectrum.

Because of the large standard deviation, the increase in MCC and accuracy, the analysis was also performed per dataset (Table 7.5). A large increase in MCC 
values was shown for the use of tissue optical features in the datasets of the disposable fiberoptic DRS probes both in vivo and ex vivo (datasets \#7 and \#9). If the tissue optical features were not taken into account, the shape-based features show the best overall results closely followed by the spectral bands. The use of peak data did not show improvement in any of the datasets.

Table 7.4: Mean increase in MCC and accuracy for all feature extraction methods compared to the use of the entire spectrum. Only not normalized data is used and only SVM and NN classifiers.

\begin{tabular}{lcc}
\hline Feature extraction & Increase in MCC (mean \pm std) & Increase in ACC (mean \pm std) \\
\hline Peak data & $-0.15 \pm 0.22$ & $-0.09 \pm 0.10$ \\
Shape-based features & $0.03 \pm 0.17$ & $0.01 \pm 0.07$ \\
Spectral bands & $-0.05 \pm 0.18$ & $-0.01 \pm 0.07$ \\
Tissue optical features & $0.25 \pm 0.28$ & $0.09 \pm 0.11$ \\
\hline
\end{tabular}

MCC $=$ Matthews Correlation Coefficient, ACC $=$ Accuracy.

As shown in section 7.4.1, since feature extraction decreased the overfitting problem for $k N N$ and LDA classifiers, the results on feature extraction were also calculated for all classifiers. For this analysis, slightly better results were obtained overall. The MCC and accuracy for the tissue optical features showed a large increase ( 0.29 and 0.09 respectively) and the shape-based features showed a smaller increase in MCC and accuracy ( 0.13 and 0.05 respectively). The results for the peak data and spectral bands improved with almost no decrease for the peak data $(\mathrm{MCC}=-0.06$ and accuracy $=-0.05$ ) and a small increase in MCC and accuracy for the spectral bands ( 0.03 and 0.02 respectively). The analysis per dataset showed similar results. In short, if possible, taking into account the fiber distance and acquisition method (fiberoptic DRS or diffuse reflection $\mathrm{HSI}$ ), tissue optical features were the best option for feature extraction. If tissue optical features could not be obtained, shape-based features were the best option.

\subsubsection{Normalization combined with feature extraction}

So far, normalization and feature extraction were only examined separately. In Table 7.6 the increase in MCC and accuracy values is shown for each combination of normalization and feature extraction compared to no feature extraction and no normalization. In the analysis of Table 7.6 all classifiers were included because using feature extraction methods decreased the risk of overfitting.

From Table 7.6, it can be concluded that independent of the normalization technique, shape-based feature extraction showed the best results. Furthermore, 
Table 7.5: Mean increase in MCC and accuracy per dataset, for all feature extraction methods compared to the use of the entire spectrum. Only non-t normalized data is used and only SVM and NN classifiers.

\begin{tabular}{|c|c|c|c|c|c|c|c|c|}
\hline & \multicolumn{2}{|c|}{ Peak data } & \multicolumn{2}{|c|}{ Shape-based } & \multicolumn{2}{|c|}{ Spectral bands } & \multicolumn{2}{|c|}{$\begin{array}{l}\text { Tissue optical } \\
\text { features }\end{array}$} \\
\hline & MCC & ACC & MCC & ACC & MCC & ACC & MCC & ACC \\
\hline \#1 & -0.27 & -0.14 & 0.04 & 0.02 & 0.06 & 0.03 & - & - \\
\hline \#2 & -0.10 & -0.05 & 0.04 & 0.01 & 0.06 & 0.03 & 0.04 & 0.02 \\
\hline$\# 3$ & -0.07 & -0.05 & 0.01 & 0.00 & 0.03 & 0.01 & - & - \\
\hline \#4 & -0.03 & -0.01 & 0.03 & 0.02 & 0.03 & 0.02 & 0 & 0 \\
\hline \#5 & -0.27 & -0.13 & 0.01 & 0.01 & -0.24 & -0.11 & - & - \\
\hline \#6 & -0.29 & -0.23 & -0.03 & 0.01 & -0.15 & -0.03 & - & - \\
\hline \#7 & 0.28 & 0.02 & 0.38 & 0.07 & 0.16 & 0.05 & 0.64 & 0.24 \\
\hline \#8 & -0.33 & -0.06 & -0.18 & -0.07 & -0.16 & -0.03 & - & - \\
\hline$\# 9$ & -0.18 & -0.17 & 0.05 & -0.02 & -0.09 & 0.02 & 0.30 & 0.08 \\
\hline
\end{tabular}

SNV normalization showed the worst results for peak data and shape-based feature extraction, but the best results for the spectral bands feature extraction. In Table 7.6, tissue optical features are not included because no normalization was applied for this feature extraction method.

In Figure 7.5, Bland-Altman plots are shown, for all datasets of which tissue optical features could be obtained and all classifiers. Separate plots were made for the combination of shape-based feature extraction with normalization at 800 $\mathrm{nm}$ (left), spectral band feature extraction and normalization at $800 \mathrm{~nm}$ (center), and the tissue optical features (right). In the Bland-Altman plot, the difference in MCC value between the combination of feature extraction and normalization, and no feature extraction and no normalization is shown. The center line indicates the mean value of all values shown in the plot and the dotted lines indicate the confidence intervals. Figure 7.5 shows that the results from the combination of shape-based feature extraction and normalization at 800 actually approached the results from the tissue optical features if only the datasets from which tissue optical features could be obtained were included.

\subsubsection{Overall results per dataset}

Based on the previous analysis on overfitting, normalization and feature extraction the best combination, obtained over all datasets, consisted of tissue optical features and an SVM or NN classifier. In case of small fiber distances or the use of the hyperspectral camera, tissue optical features were not available in some 
Table 7.6: Mean increase in MCC and accuracy over all datasets for each combination of feature extraction and normalization method compared to no normalization and no feature extraction. All classifiers are included

\begin{tabular}{llcl}
\hline & \multicolumn{3}{c}{ Increase in MCC (mean \pm std) } \\
\cline { 2 - 4 } & Peak data & Shape-based & Spectral bands \\
\hline No & $-0.04 \pm 0.25$ & $0.13 \pm 0.24$ & $0.04 \pm 0.21$ \\
SNV & $-0.07 \pm 0.26$ & $0.13 \pm 0.22$ & $0.10 \pm 0.22$ \\
AUC & $-0.07 \pm 0.24$ & $0.15 \pm 0.22$ & $0.08 \pm 0.20$ \\
At $800 \mathrm{~nm}$ & $-0.06 \pm 0.25$ & $0.16 \pm 0.23$ & $0.07 \pm 0.23$ \\
\hline \hline & & Increase in accuracy (mean \pm std) \\
\hline No & $-0.05 \pm 0.11$ & $0.05 \pm 0.09$ & $0.03 \pm 0.09$ \\
SNV & $-0.05 \pm 0.11$ & $0.04 \pm 0.09$ & $0.01 \pm 0.11$ \\
AUC & $-0.05 \pm 0.10$ & $0.04 \pm 0.09$ & $0.01 \pm 0.09$ \\
At 800 nm & $-0.05 \pm 0.12$ & $0.06 \pm 0.09$ & $0.01 \pm 0.10$ \\
\hline
\end{tabular}

$\mathrm{MCC}=$ Matthews Correlation Coefficient, ACC = Accuracy.
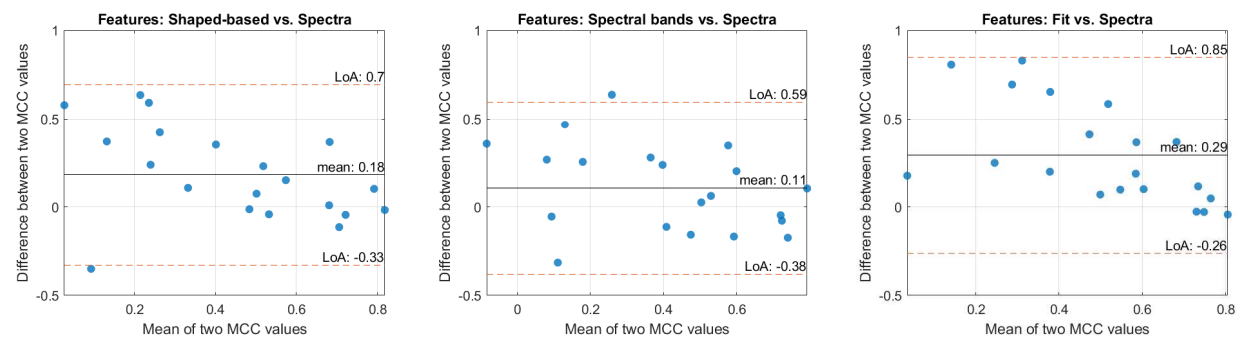

Figure 7.5: Bland-Altman plots of only the datasets of which fit-data could be obtained. From left to right the combination of shape-based feature extraction and normalization at $800 \mathrm{~nm}$, spectral bands and normalization at $800 \mathrm{~nm}$, and tissue optical features are shown. The center line indicated the average difference between the combination of feature extraction and normalization with using no normalization and no feature extraction. The average value is shown on top of the center line. The dotted lines indicate the confidence intervals.

datasets. Therefore, the shape-based features, with normalization at $800 \mathrm{~nm}$ were defined as most optimal for datasets \#1, \#3, \#5, \#6 and \#8.

For each dataset separately, the best combination of the normalization, feature extraction and classifier was evaluated based on the MCC and accuracy values. For all datasets, the best combination selected based on MCC or accuracy value were the same. In Table 7.7 an overview is given per dataset with the maximum MCC value, maximum accuracy and the normalization, feature extraction and classifier used to obtain these values.

For both datasets using disposable fiberoptic DRS needles used ex vivo (dataset \#8 and \#9), multiple combinations resulted in similar maximum MCC and accuracy values. For dataset \#8 (short distance), two combinations with similar accuracy 
and MCC value were found, based on the AUC the classification based on SVM was best, with an AUC of 0.81 for SVM compared to an AUC of 0.73 for the LDA classifier. For dataset \#9 (disposable fiberoptic DRS probe used ex vivo, long distance) three combinations of normalization, feature extraction and classifier provided the same maximum accuracies and MCC values, as shown in Table 7.7. Based on the AUC values obtained using these combinations, the combination of no normalization, tissue optical features and an LDA classifier, performed slightly better than the SNV normalized spectrum in a neural network and better than the combination of no normalization, tissue optical features in a neural network (AUC of $0.94,0.90$ and 0.84 respectively).

Table 7.7: Overview of best preforming combinations of normalization, feature extraction and classifier per dataset.

\begin{tabular}{lllllll}
\hline Data set & MCC & ACC & AUC & Normalization & Feature extraction & Classifier \\
\hline$\# 1$ & 0.88 & 0.94 & 0.96 & No & Spectral bands & SVM \\
\hline$\# 2$ & 0.84 & 0.92 & 0.98 & No & Spectral bands & SVM \\
\hline$\# 3$ & 0.84 & 0.92 & 0.95 & At $800 \mathrm{~nm}$ & Spectral bands & LDA \\
\hline$\# 4$ & 0.89 & 0.95 & 0.97 & No & Spectral bands & SVM \\
\hline$\# 5$ & 0.86 & 0.93 & 0.96 & SNV & Spectral bands & SVM \\
\hline$\# 6$ & 0.43 & 0.84 & 0.77 & At $800 \mathrm{~nm}$ & Shape-based features & NN \\
\hline$\# 7$ & 0.81 & 0.93 & 0.92 & No & Tissue optical features & SVM \\
\hline \multirow{2}{*}{$\# 8$} & 0.68 & 0.93 & 0.73 & SNV & Spectral bands & LDA \\
& 0.68 & 0.93 & 0.81 & SNV & Spectral bands & SVM \\
\hline \multirow{2}{*}{$\# 9$} & 0.68 & 0.93 & 0.94 & No & Tissue optical features & LDA \\
& 0.68 & 0.93 & 0.84 & No & Tissue optical features & NN \\
& 0.68 & 0.93 & 0.90 & SNV & Spectrum & NN \\
\hline
\end{tabular}

$\mathrm{MCC}=$ Matthews Correlation Coefficient, ACC = Accuracy, AUC = Area Under ROC Curve.

The results found in the previous section were mostly supported by the results from Table 7.7. Most classifiers found in the analysis of Table 7.7 were either SVM or NN classifiers. The two LDA classifiers that were selected both used some kind of feature reduction. For dataset \#8 and \#9, as shown in Table 7.7, the results from LDA classification were similar to the SVM and NN classifications, respectively. The same accounts for the results of dataset \#3, for which classification using shape-based features normalized with the AUC gives an MCC value of 0.81 and an accuracy of 0.90 when the SVM classifier was used.

The normalizations found in Table 7.7, were also in correspondence with previous findings in which normalization did not seem to improve the outcome. As found 
before datasets \#6 to \#9, the datasets obtained using the disposable fiberoptic probes, might benefit from normalization. This can be seen in Table 7.7 as well, where normalization for datasets \#6 to \#9 was applied in all cases were no tissue optical features were used.

For datasets \#6 to \#9, tissue optical features were selected as feature extraction technique if possible (Table 7.7), just as expected from the previous analysis. In all datasets, feature selection methods provided the best MCC values and accuracies. As predicted by the analysis on feature extraction, none of the optimal combinations used peak data as a feature extraction method.

In Table 7.8 the results of the of the optimal solution per dataset are compared to the global optimum, using SNV normalization, spectral bands as feature extraction and an SVM classifier. For the first five datasets the results of the global optimum were only slightly worse compared to the optimum of each dataset. For the final four datasets (\#6-\#9) a larger difference was seen.

Table 7.8: Results of using the global optimum combination of normalization, feature extraction and classifier (SNV+Spectral Band+SVM) compared to best-performing combinations per dataset (based on Table 7).

\begin{tabular}{|c|c|c|c|c|c|c|}
\hline \multirow[b]{2}{*}{ Dataset } & \multicolumn{3}{|c|}{$\begin{array}{c}\text { Global optimum } \\
(\mathrm{SNV}+\text { spectral band+SVM) }\end{array}$} & \multicolumn{3}{|c|}{$\begin{array}{l}\text { Dataset optimum } \\
\text { (based on Table 7) }\end{array}$} \\
\hline & MCC & ACC & $A \cup C$ & MCC & ACC & AUC \\
\hline \#1 & 0.74 & 0.86 & 0.92 & 0.88 & 0.94 & 0.96 \\
\hline \#2 & 0.78 & 0.89 & 0.97 & 0.84 & 0.92 & 0.98 \\
\hline \#3 & 0.72 & 0.86 & 0.94 & 0.84 & 0.92 & 0.95 \\
\hline \#4 & 0.85 & 0.92 & 0.96 & 0.89 & 0.95 & 0.97 \\
\hline \#5 & 0.86 & 0.93 & 0.96 & 0.86 & 0.93 & 0.96 \\
\hline$\# 6$ & 0.13 & 0.57 & 0.60 & 0.43 & 0.84 & 0.77 \\
\hline \#7 & 0.52 & 0.72 & 0.81 & 0.81 & 0.93 & 0.92 \\
\hline \#8 & 0.68 & 0.93 & 0.81 & 0.68 & 0.93 & 0.81 \\
\hline$\# 9$ & 0.52 & 0.89 & 0.88 & 0.68 & 0.93 & 0.94 \\
\hline
\end{tabular}

To confirm the results obtained with the holdout test, performed in this research, the test results of the holdout test were compared to a 20 -fold cross-validation. In Table 7.9 the results of this comparison are shown. The results are shown for the combination of SNV normalization, spectral band feature extraction and an SVM classifier. As can be seen in Table 7.9 only minor differences were shown between the holdout test results and the 20 -fold cross-validation. 
Table 7.9: Comparison between the performance of cross-validation and holdout validation.

\begin{tabular}{cccccccc}
\hline & \multicolumn{3}{c}{ Holdout $(20 \%-80 \%)$} & & \multicolumn{3}{c}{ 20-fold cross-validation } \\
\cline { 2 - 3 } \cline { 7 - 8 } Dataset & MCC & ACC & AUC & & MCC & ACC & AUC \\
\hline$\# 1$ & 0.74 & 0.87 & 0.92 & & 0.75 & 0.88 & 0.93 \\
$\# 2$ & 0.78 & 0.89 & 0.97 & & 0.75 & 0.88 & 0.95 \\
$\# 3$ & 0.72 & 0.86 & 0.93 & & 0.63 & 0.82 & 0.86 \\
$\# 4$ & 0.84 & 0.92 & 0.96 & & 0.81 & 0.91 & 0.95 \\
$\# 5$ & 0.85 & 0.93 & 0.96 & & 0.78 & 0.89 & 0.95 \\
\hline
\end{tabular}

\subsection{Discussion}

In the current study, an optimal analysis technique was examined to distinguish healthy colorectal wall from tumor tissue. The analysis was done on nine different datasets, using five different classifiers, three normalization techniques and four feature extraction methods, compared to the use of the entire spectrum without normalization. Based on these analyses it was found that the best overall combination is to use no normalization, an SVM or NN as a classifier and as feature extraction method the tissue optical features or the shape-based features.

\subsubsection{Overfitting}

The results on overfitting show a large chance of overfitting for $k N N$, decision tree and LDA classifiers if the entire spectrum was used. This was expected because the number of features used for the classification is large compared to the number of samples for each dataset. As a rule of thumb the number of slightly correlated features should not exceed the square root of the number of samples [54]. For the use of the entire spectrum, this rule of thumb is not met which increases the chance of overfitting. It was shown that the decrease in the number of features used for the classification resulted in a decrease in overfitting (Figure 7.4c). No improvement in overfitting was seen when datasets were normalized (Figure 7.4b). This was expected because normalization does not change the number of features, neither does it decrease the presence of noise in the data. For the SVM and NN classifiers overall overfitting was less present. Therefore, it was decided to continue with these two classifiers for further analysis. 


\subsubsection{Normalization}

Overall, normalization does not seem to improve classification outcomes (Table 7.2). However, if datasets are evaluated separately a trend is seen when datasets obtained with the reusable fiberoptic DRS probe (\#1 to \#4) are compared to datasets obtained with the disposable fiberoptic DRS probe (\#6 to \#9). For the first four datasets, normalization does not seem to improve the results. On the contrary, for the later four normalization does improve the results. This might have to do with the use of disposable needles in the second group (datasets \#6 to \#9). Even though manufacturing of these probes was automated, small differences in fiber distances were present over the set of probes. Furthermore, for the white reference of the final four datasets a separate probe was used, which was not the case for the first four datasets. The use of a new probe for each patient and the use of a separate white reference probe, which required changing the fibers between calibration and measurement, might have caused intensity differences in the final four datasets which were not tissue specific. The intensity differences caused by both issues, will be eliminated by normalization of the spectra. However, normalization might also eliminate tissue specific intensity differences. This is probably the reason why normalization is not improving the results for the first four datasets in which the measurement set-up is more constant.

For diffuse reflection HSI, SNV normalization is often used because it removes intensity differences due to glare [47]. However, as shown in Table 7.3, SNV normalization does not improve the results of the diffuse reflection HSI data, neither did the other two normalization techniques. Apparently, glare does not affect the features vital to the classification. Even though in general SNV does not seem to improve the analysis of diffuse reflection HSI significantly, the combination resulting in the best accuracy and MCC for the diffuse reflection HSI dataset did use SNV normalization (Table 7.7).

\subsubsection{Feature extraction}

Because of the chances of overfitting when using the entire spectra for classification different feature extraction methods were examined. The use of information from the seven most prominent peaks of the spectra does not give any useful information. As shown in Table 7.4 and Table 7.5 the MCC and accuracy values decrease when using the peak data compared to the use of the entire spectrum. An advantage of using peak data compared to the entire spectrum is the fact that 
less features are used in the classification, 21 features for the peak data compared to 1151 features for the entire spectrum. Even though the use of peak data is not the most optimal feature extraction method, the decrease in the number of features used, compared to the use of the entire spectrum, does show an advantage.

The best feature extraction method is the use of tissue optical features. However, the use of tissue optical features has some restrictions compared to the other feature extraction methods. First of all, the outcome of the fit algorithm depends largely on the tissue constituents that are given as input to the fit algorithm. This means, that if not all tissue constituents are known, the results will be unreliable. Furthermore, several assumptions are made for the fit model. The model which has been used in this paper is designed only for fiber optics with fiber distances larger than $1 \mathrm{~mm}$. This algorithm does not work properly on hyperspectral data as well as on the spectra from fibers with less than $1 \mathrm{~mm}$ distance. To best of our knowledge there is no generalized fit algorithm that could work properly for all datasets. The use of shape-based features seems the second best option, which can be used for all datasets. The results of the shape-based feature could be improved by adding more features to better describe the shape of the spectra and more importantly describe the difference between the two tissue types.

\subsubsection{General optimum}

As stated before, there is not one best model that works for every problem. However, in Table 7.8 it is shown that if a global optimum, the SNV normalization, spectral bands and an SVM classifier, was compared to the optimum of each dataset, only small differences were seen for the first five datasets. Datasets \#6-\#9 did show large differences between the optimum of each dataset and the global optimum. This can be explained by the use of the disposable needles, which has less influence on the extraction of the tissue optical features compared to the other feature extraction methods. Furthermore, when the conclusions of the separate analyses are compared to the optimal solution for each dataset (Table 7.7), there is a large resemblance. For instance, in the final four datasets, normalization is used for all datasets in which no tissue optical features was used as feature extraction method, whereas for the other fiberoptic DRS dataset no normalization was used except for dataset \#3 (Table 7.7). Furthermore, for all datasets feature extraction was used. Overall, in almost all datasets the optimal 
classifier is either a SVM or a NN. So even though there is no one best model, some rules could be set to optimize the classification of DRS and HSI data in colorectal cancer. However, generalization of these rules to other tissue types or optical technologies cannot be done without examination first.

\subsubsection{Future work}

The current research did only cover 6 basic classifiers. Future research should also include more state of the art classifiers like a random forest classifier and a convolutional NN. Furthermore, the option of boosting should be investigated to further optimize the classification. For the hyperspectral images, classification could be further optimized by taking into account the spatial information. However, with this also the dependency of neighboring pixels should be considered. In the current hyperspectral dataset the dependency of the neighboring pixels was not taken into account. Furthermore, to overcome the possibility of the influence of multiple tissue types on one pixel, pixels at the border between two tissue types were removed from the dataset.

\subsection{Conclusion}

From the present study it can be concluded that normalization does not seem to have added value, except to remove differences originating from the use of different DRS probes within one dataset. Furthermore, in a clinical setting where the number of samples is often limited, the use of feature extraction methods is needed to prevent overfitting in high dimensional data classification. On the other hand, the results of feature extraction could be helpful to design simpler devices focused on measuring only the features we are interested in rather than the full spectrum, like multispectral cameras. Finally, SVM and NN are the best performing classifiers with the least chance of overfitting on these datasets. 


\section{References}

[1] F. Bray et al., "Global cancer statistics 2018: GLOBOCAN estimates of incidence and mortality worldwide for 36 cancers in 185 countries," CA: A Cancer Journal for Clinicians, vol. 68, pp. 394424, nov 2018.

[2] K. D. Miller et al., "Cancer treatment and survivorship statistics, 2016," CA: A Cancer Journal for Clinicians, vol. 66, pp. 271-289, jun 2016.

[3] S. G. Brouwer de Koning et al., "Toward complete oral cavity cancer resection using a handheld diffuse reflectance spectroscopy probe," Journal of Biomedical Optics, vol. 23, pp. 1-9, oct 2018.

[4] L. L. Boer et al., "Fat/water ratios measured with diffuse reflectance spectroscopy to detect breast tumor boundaries," Breast Cancer Research and Treatment, vol. 152, no. 3, pp. 509-518, 2015.

[5] J. W. Spliethoff et al., "Improved identification of peripheral lung tumors by using diffuse reflectance and fluorescence spectroscopy," Lung Cancer, vol. 80, no. 2, pp. 165-171, 2013.

[6] G. C. Langhout et al., "Differentiation of healthy and malignant tissue in colon cancer patients using optical spectroscopy: A tool for image-guided surgery," Lasers in Surgery and Medicine, vol. 47, no. 7, pp. 559-565, 2015.

[7] R. M. Schols, P. Dunias, F. P. Wieringa, and L. P. S. Stassen, "Multispectral characterization of tissues encountered during laparoscopic colorectal surgery," Medical Engineering and Physics, vol. 35, no. 7, pp. 1044-1050, 2013.

[8] J. S. Soares et al., "Diagnostic power of diffuse reflectance spectroscopy for targeted detection of breast lesions with microcalcifications," Proceedings of the National Academy of Sciences, vol. 110, pp. $471 \mathrm{LP}-476$, jan 2013.

[9] B. Fei et al., "Label-free reflectance hyperspectral imaging for tumor margin assessment: a pilot study on surgical specimens of cancer patients," Journal of Biomedical Optics, vol. 22, p. 1, aug 2017.

[10] G. Lu and B. Fei, "Medical hyperspectral imaging: a review," Journal of Biomedical Optics, vol. 19, p. 10901, jan 2014.

[11] R. Nachabé et al., "Estimation of biological chromophores using diffuse optical spectroscopy: benefit of extending the UV-VIS wavelength range to include 1000 to $1600 \mathrm{~nm}$," Biomedical Optics Express, vol. 1, no. 5, pp. 1432-1442, 2010.

[12] K. Masood and N. M. N. M. Rajpoot, "Classification of colon biopsy samples by spatial analysis of a single spectral band from its hyperspectral cube," 2007.

[13] K. Masood and N. M. N. M. Rajpoot, "Spatial analysis for colon biopsy classification from hyperspectral imagery," 2008.

[14] K. Rajpoot and N. Rajpoot, "SVM Optimization for Hyperspectral Colon Tissue Cell Classification," in Medical Image Computing and Computer-Assisted Intervention - MICCAI 2004 (C. Barillot, D. R. Haynor, and P. Hellier, eds.), (Berlin, Heidelberg), pp. 829-837, Springer Berlin Heidelberg, 2004. 
[15] S. Rathore, M. Hussain, A. Ali, and A. Khan, "A Recent Survey on Colon Cancer Detection Techniques," IEEE/ACM Transactions on Computational Biology and Bioinformatics, vol. 10, no. 3 , pp. 545-563, 2013.

[16] M. Maggioni et al., "Hyperspectral microscopic analysis of normal, benign and carcinoma microarray tissue sections," vol. 6091, p. 60910I, International Society for Optics and Photonics, feb 2006.

[17] E. Claridge and D. Hidović-Rowe, "Model Based Inversion for Deriving Maps of Histological Parameters Characteristic of Cancer From Ex-Vivo Multispectral Images of the Colon," IEEE Transactions on Medical Imaging, vol. 33, no. 4, pp. 822-835, 2014.

[18] Z. Han et al., "In vivo use of hyperspectral imaging to develop a noncontact endoscopic diagnosis support system for malignant colorectal tumors," vol. 21, pp. 16001-16008, 2016.

[19] R. Kumashiro et al., "Integrated Endoscopic System Based on Optical Imaging and Hyperspectral Data Analysis for Colorectal Cancer Detection," Anticancer Research, vol. 36, pp. 3925-3932, aug 2016.

[20] E. J. M. Baltussen et al., "Hyperspectral imaging for tissue classification, a way toward smart laparoscopic colorectal surgery," Journal of Biomedical Optics, vol. 24, pp. 1-9, jan 2019.

[21] G. Zonios et al., "Diffuse reflectance spectroscopy of human adenomatous colon polyps in vivo," Applied optics, vol. 38, no. 31, pp. 6628-6637, 1999.

[22] H.-W. Wang et al., "Diffuse reflectance spectroscopy detects increased hemoglobin concentration and decreased oxygenation during colon carcinogenesis from normal to malignant tumors.," Optics express, vol. 17, no. 4, pp. 2805-17, 2009.

[23] H. K. Roy et al., "Spectroscopic Microvascular Blood Detection From the Endoscopically Normal Colonic Mucosa: Biomarker for Neoplasia Risk," Gastroenterology, vol. 135, no. 4, pp. 10691078, 2008.

[24] A. Dhar et al., "Elastic scattering spectroscopy for the diagnosis of colonic lesions: initial results of a novel optical biopsy technique," Gastrointestinal Endoscopy, vol. 63, pp. 257-261, feb 2006.

[25] J. R Mourant et al., Elastic scattering spectroscopy as a diagnostic tool for differentiating pathologies in the gastrointestinal tract: Preliminary testing, vol. 1. apr 1996.

[26] E. J. M. Baltussen et al., "Diffuse reflectance spectroscopy as a tool for real-time tissue assessment during colorectal cancer surgery," Journal of biomedical optics, vol. 22, no. 10, pp. 106014106016, 2017.

[27] T. M. Bydlon et al., "Chromophore based analyses of steady-state diffuse reflectance spectroscopy: current status and perspectives for clinical adoption," Journal of Biophotonics, vol. 8, pp. 9-24, jan 2015.

[28] R. Nachabé et al., "Estimation of lipid and water concentrations in scattering media with diffuse optical spectroscopy from 900 to $1,600 \mathrm{~nm}$.," Journal of biomedical optics, vol. 15, no. 3, p. 037015, 2015. 
[29] R. M. Schols et al., "Differentiation between nerve and adipose tissue using wide-band $(350-1,830 \mathrm{~nm})$ in vivo diffuse reflectance spectroscopy," Lasers in surgery and medicine, vol. 46, no. 7, pp. 538-545, 2014.

[30] J. W. Spliethoff et al., "Real-time in vivo tissue characterization with diffuse reflectance spectroscopy during transthoracic lung biopsy: a clinical feasibility study," Clinical Cancer Research, vol. 22, no. 2, pp. 357-365, 2015.

[31] J. W. Spliethoff et al., "In vivo characterization of colorectal metastases in human liver using diffuse reflectance spectroscopy: toward guidance in oncological procedures," Journal of Biomedical Optics, vol. 21, no. 9, p. 97004, 2016.

[32] E. Tanis et al., "Real-time in vivo assessment of radiofrequency ablation of human colorectal liver metastases using diffuse reflectance spectroscopy," European Journal of Surgical Oncology (EJSO), vol. 42, no. 2, pp. 251-259, 2016.

[33] L. L. de Boer et al., "Using DRS during breast conserving surgery: identifying robust optical parameters and influence of inter-patient variation," Biomedical optics express, vol. 7, no. 12, pp. 5188-5200, 2016.

[34] R. Nachabé et al., "Real-time in vivo characterization of primary liver tumors with diffuse optical spectroscopy during percutaneous needle interventions: feasibility study in woodchucks," Investigative radiology, vol. 50, no. 7, pp. 443-448, 2015.

[35] R. Nachabé et al., "Diagnosis of breast cancer using diffuse optical spectroscopy from 500 to $1600 \mathrm{~nm}$ : comparison of classification methods," Journal of biomedical optics, vol. 16, no. 8, p. 87010, 2011.

[36] D. J. Evers et al., "Optical sensing for tumor detection in the liver," European Journal of Surgical Oncology, vol. 39, no. 1, pp. 68-75, 2013.

[37] B. H. W. Hendriks et al., "Nerve detection with optical spectroscopy for regional anesthesia procedures," Journal of Translational Medicine, vol. 13, p. 380, 2015.

[38] G. Lu et al., "Detection of Head and Neck Cancer in Surgical Specimens Using Quantitative Hyperspectral Imaging," Clinical Cancer Research, vol. 23, pp. 5426 LP - 5436, sep 2017.

[39] M. P. L. Bard et al., "Improving the specificity of fluorescence bronchoscopy for the analysis of neoplastic lesions of the bronchial tree by combination with optical spectroscopy: preliminary communication," Lung Cancer, vol. 47, no. 1, pp. 41-47, 2005.

[40] D. J. Evers et al., "Diffuse reflectance spectroscopy: a new guidance tool for improvement of biopsy procedures in lung malignancies," Clinical lung cancer, vol. 13, no. 6, pp. 424-431, 2012.

[41] Z. Liu, H. Wang, and Q. Li, "Tongue Tumor Detection in Medical Hyperspectral Images," Sensors (Basel, Switzerland), vol. 12, pp. 162-174, dec 2012.

[42] L. L. De Boer et al., "Towards the use of diffuse reflectance spectroscopy for real-time in vivo detection of breast cancer during surgery," Journal of translational medicine, vol. 16, no. 1, p. 367 , 2018. 
[43] H. Chen et al., "Near-infrared spectroscopy as a diagnostic tool for distinguishing between normal and malignant colorectal tissues," BioMed research international, vol. 2015, 2015.

[44] R. J. Barnes, M. S. Dhanoa, and S. J. Lister, "Standard normal variate transformation and de-trending of near-infrared diffuse reflectance spectra," Applied spectroscopy, vol. 43, no. 5 , pp. 772-777, 1989.

[45] A. Rinnan, F. van den Berg, and S. B. Engelsen, "Review of the most common pre-processing techniques for near-infrared spectra," TrAC Trends in Analytical Chemistry, vol. 28, pp. 12011222, nov 2009.

[46] E. Kho, B. Dashtbozorg, L. L. de Boer, K. K. V. de Vijver, H. J. C. M. Sterenborg, and T. J. M. Ruers, "Hyperspectral imaging for resection margin assessment during cancer surgery,"Biomed. Opt. Express, vol. 10, pp. 4496-4515, 2019.

[47] E. Kho et al., "Hyperspectral imaging for resection margin assessment during cancer surgery," Clinical Cancer Research, pp. clincanres-2089, 2019.

[48] R. O. Duda, P. E. Hart, and D. G. Stork, "Pattern classification," (John Wiley \& Sons, 2012).

[49] C. M. Bishop, Pattern recognition and machine learning. springer, 2006.

[50] T. J. Farrell, M. S. Patterson, and B. Wilson, "A diffusion theory model of spatially resolved, steady-state diffuse reflectance for the noninvasive determination of tissue optical properties in vivo," Medical Physics, vol. 19, no. 4, pp. 879-888, 1992.

[51] R. Nachabe et al., "Estimation of lipid and water concentrations in scattering media with diffuse optical spectroscopy from 900 to 1600 nm," vol. 15, pp. 37010-37015, 2010.

[52] D. H. Wolpert and W. G. Macready, "No free lunch theorems for optimization," IEEE transactions on evolutionary computation, vol. 1, no. 1, pp. 67-82, 1997.

[53] S. Boughorbel, F. Jarray, and M. El-Anbari, "Optimal classifier for imbalanced data using Matthews Correlation Coefficient metric," PloS one, vol. 12, no. 6, p. e0177678, 2017.

[54] E. Suh et al., "Optimal number of features as a function of sample size for various classification rules," Bioinformatics, vol. 21, pp. 1509-1515, nov 2004. 
Chapter 8

General Discussion 
In this thesis, two optical techniques were examined to discriminate healthy tissue from tumor tissue in colorectal cancer surgery to reduce positive resection margins. Fiberoptic diffuse reflectance spectroscopy (DRS) and diffuse reflection hyperspectral imaging $(\mathrm{HSI})$ rely on the same principal of the interaction of light with tissue. However, due to the difference in measurement set-up the ultimate use of both techniques differs, as well as the challenges that still need to be resolved before both techniques will be used in the clinic. In this thesis several steps have been made for both techniques toward the ultimate clinical use. In addition, this thesis describes several challenges that still need to be resolved, some that apply to both techniques and some technique specific. These challenges will be discussed in this chapter.

\subsection{Fiberoptic diffuse reflectance spectroscopy}

As stated in Chapter 1, the final application of fiberoptic DRS would be to incorporate the technique into a surgical device. When using this device the surgeon would know what tissue type is in front of the device and will be able to act on this information. In the development of such a device several steps need to be made. First, the ability of fiberoptic DRS to distinguish tumor from healthy surrounding tissues encountered during colorectal cancer surgery should be examined. This was done in the most controlled way in Chapter $\mathbf{2}$ by performing fiberoptic DRS measurements on tissue samples. In this study it was found that fiberoptic DRS is capable of discriminating tumor, fat and healthy colorectal wall with accuracies of $0.92,1.00$ and 0.93 respectively. These findings were supported by previous ex vivo research done in colorectal cancer [1, 2].

\section{Fibrosis}

The study of Chapter $\mathbf{2}$ did not included fibrosis as a healthy tissue type. However, in locally advanced rectal cancer, patients more often receive (chemo)radiotherapy before surgery. This induces fibrosis around the tumor area. Therefore, it is important to be able to discriminate tumor from fibrosis using fiberoptic DRS. This next step was taken in Chapter $\mathbf{4}$, where measurements were performed on entire rectal specimen. In this study, it was found that it is possible to distinguish tumor from fibrosis if measurements contained only fibrosis or only tumor, e.g. if the measurements were on pure tissue types. When these measurements were clas- 
sified, an accuracy of 0.88 was obtained. However, when measurements were included in which tumor was covered with a layer of healthy tissue, mainly fibrosis, the classification accuracy decreased to 0.61 and the Matthews correlation coefficient (MCC) decreased to 0.17 , which is not much better than random $(\mathrm{MCC}=0$ ). These results were supported by the mean spectra shown in 4.4. Where the mean spectra of pure tumor and pure fibrosis can be distinguished, it is harder to distinguish the spectra obtained on tumor with a layer of fibrosis on top from each other and from pure fibrosis.

There are several possible explanations for this decrease in accuracy between the classification of pure measurements and mixed measurements. A possible explanation for the decrease in accuracy is the reduced amount of tumor in the measurements volume of the mixed measurements. In Chapter 2, it was already shown that classification of border locations was less accurate compared to measurements with just one tissue type, with accuracies of 0.80 and 0.95 respectively. The border locations are similar to the measurements from Chapter 4, in which tumor is covered with a layer of healthy tissue. As stated in Chapter 2, these locations will be classified as the most prominent tissue type present in the measurement volume. For the measurements in Chapter 4, about half of the measurements included with the label tumor have a layer of 2-3 $\mathrm{mm}$ healthy tissue on top of tumor. Even though the fiber distance of $2 \mathrm{~mm}$ would provide a measurement depth of about $2 \mathrm{~mm}$, the majority of the measured volume will consist of healthy tissue. This will cause the classifier to classify these measurements as healthy. The sensitivity of the fiberoptic DRS for tumor will probably increase if the healthy layer on top of tumor would be less than $1 \mathrm{~mm}$ thick. Especially when the fiber distance is $2 \mathrm{~mm}$, the measured volume of tumor will increase. However, because there is no depth information in the fiberoptic DRS measurements, it will not be known at which depth the tumor is present. Decreasing the fiber distance to 1 $\mathrm{mm}$, will decrease the measurement depth and will thereby probably not increase the sensitivity of fiberoptic DRS for tumor at a depth of $1 \mathrm{~mm}$.

The classification outcome might be improved by adding more measurements with sufficient tumor volume. However, because the surgeon will try to prevent positive resection margins, the chances of measuring tumor at the resection surface of a rectum specimen are small. Furthermore, even if there is tumor at the resection surface, these areas shall be very small, in the order of millimeters. In combination with a measurement surface of 2-3 $\mathrm{mm}$, the chances of measuring tumor at the surface seem small. Measuring the entire resection surface of the 
rectum using the fiberoptic probe is not possible due to time limitations and because registration with pathology cannot be made for the entire specimen. Therefore, other options should be explored to locate the most useful measurement locations in a specimen. In Chapter 4, ultrasound was already used. However, in ultrasound images no discrimination can be made between tumor and fibrosis. It is therefore hard to locate tumor locations using ultrasound images. Another option would be to make use of a contrast agent to locate the tumor region. One option would be to administer a molecular targeted fluorescent dye, which highlights the tumor region and with this the most useful measurement area [4]. It should be noted that the fluorescent dye could influence the optical signals detected with fiberoptic DRS. The fluorescent dye would only be needed during training of the classification. After training, the algorithm should be able to perform well without the use of fluorescence.

\section{Circumferential resection margin}

In rectal cancer surgery the circumferential resection margin (CRM) is defined as the shortest distance between the tumor and the resected surface. A positive CRM is defined as tumor within $1 \mathrm{~mm}$ of the resected surface and is a negative independent predictor of local recurrence and overall survival $[5,6]$. When fiberoptic DRS is going to be used during rectal cancer surgery it should thus be able to detect tumor at a distance of $1 \mathrm{~mm}$ below the measurement surface. The measurement depth of fiberoptic DRS is dependent on the fiber distance and is approximately the same as the fiber distance [7,8]. This is supported by the results in Chapter 5, Figure 5.8. Here, the accuracy and the MCC are shown for an increasing maximum depth of tumor. The maximum accuracy and MCC value are reached with a maximum depth of the tumor of $1.5 \mathrm{~mm}$. For this study a fiber distance of $1.29 \mathrm{~mm}$ was used, which is in agreement with the maximum accuracy and MCC. In this analysis only fat and healthy colorectal wall covered tumor. In Chapter $\mathbf{4}$ it was found that attempting to detect tumor below a layer of fibrosis resulted in low accuracies, as discussed in the previous section.

The difference between the results from Chapter $\mathbf{4}$ and Chapter 5 could be due to the fiber distance or due to the tissue layer covering the tumor. With the smaller fiber distance used in Chapter 5, the measurement volume was smaller and thus probably was more homogeneous compared to the larger measurement volume measured with the larger fiber distance used in Chapter 4. With the smaller fiber distance there is more chance of measuring just one layer instead of different 
layers at larger depths. This will improve the classification accuracy because it is more easy to classify measurements on pure tissue compared to layered tissue. A second explanation for the different results in Chapter $\mathbf{4}$ and Chapter $\mathbf{5}$ could be the tissue type covering the tumor. If fibrosis is more similar to tumor compared to healthy colorectal wall, it is more difficult to detect tumor below a layer of fibrosis than to detect tumor below a layer of healthy colorectal wall. If this was the reason, we could solve it by adding measurements with a second, smaller, fiber distance. In this case, two measurements will be obtained on one location, one with a small fiber distance and one with a larger fiber distance. The first, will provide information on the top layer. The latter, will provide information from larger depths and will be the same as was measured with just one fiber distance. Because there is information about the top layer from the small fiber distance, more information might be extracted from the measurement at larger depth. This might not only be useful to determine the CRM, but also to get a better idea of the difference between tumor and fibrosis, even if no pure tumor or fibrosis measurements can be obtained.

\subsection{Diffuse reflection hyperspectral imaging}

In Chapter 3, tissue samples were imaged using two hyperspectral cameras. It was found that diffuse reflection $\mathrm{HSI}$ can be used to distinguish between fat, healthy colorectal wall and colorectal cancer tissue. Similar results are obtained as in Chapter 2 using fiberoptic DRS, with a mean accuracy of 0.88 over all tissue types. For diffuse reflection HSI the next steps, imaging of entire specimen and applying the technique in vivo, have not been made yet. Several issues should first be resolved before these steps can be made.

\section{Diffuse reflection HSI of entire specimen}

Chapter 3 describes the imaging of tissue samples using diffuse reflection HSI. The step to imaging entire specimen is not described in this thesis, but the first few specimen have already been imaged. For this research the rectum specimen that were used in Chapter 4 were imaged using the two hyperspectral cameras used in Chapter 3. A few challenges are encountered during the imaging of the entire specimen, but the main challenge is the correlation with pathology. When imaging the tissue samples (Chapter 3), pathological classification of the entire 
sample was obtained, as shown in Figure 3.2. It is however, not possible to obtain pathological classification of the surface of an entire specimen. The pathology assessment of rectum specimen is focused on the CRM. Therefore, the rectum specimens are sliced into transverse sections of about $5 \mathrm{~mm}$ thick [9]. For this research 3-4 of these cross-section rings can be marked and thereafter used for pathological correlation. So, pathology information of about $1.5-2.0 \mathrm{~cm}$ of the surface is known. In Chapter 4, it is found that the surface area of positive CRMs in the rectal specimen is at most $1 \mathrm{~cm}$ and often discontinues. These small areas of tumor at the surface of a resected specimen are not easy to find. Locating these areas within the $1.5-2.0 \mathrm{~cm}$ that will be evaluated by the pathology will be even more difficult.

A first solution to this problem would be to classify the hyperspectral images realtime to locate the positive CRM. If a positive CRM is then located, this area can be marked for further pathological evaluation. A first step would be to apply the classification model created in Chapter 3, to the images of the entire specimen. This was tried for the few specimen that were imaged. However, it was found that the classification could correctly classify fat, but for all other tissue types low accuracies were obtained. An explanation for the mediocre performance of this classifier developed on slices when applied to entire specimen is the difference in spectra. As was found by Kho et al., when thin $(3-5 \mathrm{~mm})$ slices are imaged using a hyperspectral camera, part of the light might penetrate the entire tissue sample as well as the matter below the tissue sample. All tissue samples in Chapter 3 were imaged with a slice of black rubber below the tissue sample. This rubber absorbs the light that exits the tissue sample at the bottom, without adding specific characteristics to the spectra. However, even though the rubber does not add a certain characteristic to the obtained spectra, it will alter the spectra by absorbing the light that penetrated the tissue samples. These alteration will not be present when an entire specimen is imaged, as these specimens are a few centimeters thick. Furthermore, in Chapter $\mathbf{3}$ cross-sections of the tumor are imaged. These tumor areas might differ from the tumor at the surface of the resected specimen. The tumor at the surface of the resected specimen will most likely be a small area with a mix of tumor and healthy tissue, whereas the majority of the tumor from the cross-sections was pure tumor.

To solve these problems a classification based on data obtained on entire specimen should be made. To create this classification, data from entire specimen are needed of which also pathology labels are available. As pathology can only be ob- 
tained from $1.5-2.0 \mathrm{~cm}$ of the surface, the examined area should be picked careful in order to include enough tumor locations. Again, the use of a molecular targeted contrast agent could be a solution for the training of the classifier, using a fluorescent dye to locate tumor close to the resection margin and marking this area for pathology evaluation will lead to more tumor locations in the train dataset. Another option would be to locate the tumor at the pathology department and make one transverse cut through the tumor. Thereby, a large surface area of tumor can be imaged with the hyperspectral camera. This solves the problem of the limited surface area of tumor at the surface of the resected specimen. It also solves the problem of the slices thickness, because in contrast to the slices scanned before, now the cross section of the dissected specimen is imaged. However, there might still be a difference between the tumor detected at the cross-section and the tumor detected at the surface. Based on data from 5-10 specimen it should be examined whether there is a difference between the signals obtained from the cross-sections and signals obtained from the surface. If there is no difference, the cross-section images could be used to create a database for a first classification. This classification can be used to classify the surface of the new resected specimen. Furthermore, the classification can be updated with each specimen that is imaged. Each new dataset can be added to the train data, where the classification of each pixel should be adjusted according to the pathology classification.

\section{Spatial information}

One of the advantages of using diffuse reflection HSI over fiberoptic DRS is the addition of spatial information. In this thesis the spatial information from the hyperspectral images was not used. The classification performed in Chapter $\mathbf{3}$ is based on the spectral information only. Training a classification on the spatial information from the tissue sample in Chapter 3 does not seem useful as the tissue samples are far from representative of what the tissue, assessed during surgery, will look like. However, for the classification of the entire specimen, spatial information might be of added value. The training of a classification that uses both spectral and spatial information should be done on the surface of the resected specimen to train the classifier correctly. 


\section{Measurement set-up}

In the current measurement set-up of the hyperspectral cameras as used in Chapter 3 , the tissue needs to be placed on a translation stage, which moves the tissue underneath one of the cameras. To image both the visual and near-infrared wavelength range, the tissue should be moved from one translation stage to another. If diffuse reflection $\mathrm{HSI}$ is going to be used in vivo, changes are needed in the measurement set-up. As described in Chapter 1, there are several different ways to obtain a hyperspectral image, spatial scanning, spectral scanning, spatiospectral scanning and snapshot imaging. In contrast to spatial scanning where the tissue is moved with respect to the camera, in spectral scanning the tissue should be fixed with respect to the camera while the filters in front of the camera change to obtain the entire desired wavelength range. Both methods do not seem usable for an in vivo setting [10]. Spectral scanning could work if only a selected number of wavelengths is selected. However, to obtain the full wavelength range acquisition time will be long and the tissue will probably move with respect to the camera during acquisition. In spatiospectral scanning an element within the camera can be moved to obtain the entire 3D data cube. However, here again the tissue sample should be fixed with respect to the camera in order to obtain a correct 3D data cube, making this only possible for a selected number of wavelengths [11]. The snapshot camera could be a solution for these problems, because this camera obtains a 3D data cube at once. However, large computational expenses, because the entire data cube is obtained at once, and large costs are associated with such a snapshot camera [12]. Another option would be to use a multispectral snapshot camera, in which several wavelength are selected to improve measurement speed and decrease computational expenses. In order to make full use of a multispectral camera, the most useful wavelengths for tissue discrimination should be selected based on the hyperspectral images. Furthermore, the most discriminating wavelengths will differ for different applications, which limits the use of these cameras.

\subsection{General}

Besides the technique specific challenges, there are several challenges that apply to both techniques. Most are related to data processing and analysis of the data. 


\section{In vivo versus ex vivo}

In Chapter 6, it was examined whether there is a difference between fiberoptic DRS measurements obtained in an ex vivo setting and measurements obtained in an in vivo setting. Even though this was only examined for fiberoptic DRS, the same issue exists in diffuse reflection HSI. Performing measurements in an ex vivo setting is more controlled and it is easier to included subjects compared to in vivo. Creating an ex vivo dataset is therefore easier compared to an in vivo dataset, resulting in larger datasets for ex vivo studies. In Chapter 6, differences between in vivo and ex vivo measurements were examined based on fit parameters. The switch to fit parameters instead of using the entire spectrum or spectral bands, as is done in all other chapters of this thesis, is because with the use of fit parameters, changes can be related to biological phenomena.

In Chapter 6 it is found that a only the intensity scale factor and the diameter of the blood vessels do not differ significantly between in vivo and ex vivo measurements for all tissue types. Despite the differences between in vivo and ex vivo fit parameters, the classification of the tissue types is not influenced significantly. No significant difference is found between the classification that is trained and tested on in vivo data and the classification that is trained on ex vivo data and tested on in vivo data. A possible explanation for this is, that the parameters on which the classification is mostly based, change in the same direction for all tissue types between in vivo and ex vivo. Based on the results from Chapter 6 one can conclude that a tissue classification can be trained on ex vivo data and thereafter used in vivo, at least for optical measurements in colorectal cancer. Care should be taken in generalizing these results to different tissue types, as processes like dehydration might differ per tissue type.

\section{Analysis techniques}

In this thesis several different analysis techniques were used. In Chapter 2, spectra are not normalized, spectral bands are used to reduce the number of features used for classification, and a quadratic classifier and an SVM are used for classification. In Chapter 3, spectra are normalized using standard normal variate (SNV) normalization, with otherwise similar analysis as in Chapter 2. Normalization in Chapter $\mathbf{4}$ is done by setting the area under the curve (AUC) for each spectrum to one. Classification is performed using just one SVM on the entire spectra, because only two different tissue types needed to be separated. In Chapter $\mathbf{5}$, 
spectra are normalized at $800 \mathrm{~nm}$, and classification, on the entire spectra, is performed using two SVMs. Finally, in Chapter 6, fit parameters are used for classification using three, one versus one, SVMs. In all chapters a different choice in normalization, feature extraction and classifier is made. In Chapter $\mathbf{7}$, an analysis is done on the different normalization, feature extraction and classification methods for healthy colorectal wall versus tumor.

\section{Normalization}

Normalization of diffuse reflection HSI data using SNV normalization was assumed to be necessary to remove the influence of glare from the spectra. However, in Chapter $\mathbf{7}$ it is shown that overall SNV normalization does not improve the outcome for classification in the diffuse reflection HSI dataset. On the contrary, SNV normalization seems the worst performing normalization technique. This might have to do with the fact that SNV normalization not only removes the influence of glare from the spectra but also removes the effects of scattering. In fiberoptic DRS, normalization also does not improve classification results as shown in Chapter 7. Except, in the datasets obtained in the in vivo study, for which normalization does improve the classification results. There are two possible explanations for this result. First, in this study a different needle is used for each patient. If the fiber distances in these needles were slightly different, differences in the measured spectra might appear, which are not tissue specific but are caused by differences between the needles.

Second, to keep the needle sterile until the in vivo measurements, the white reference was performed with a different needle. In each patient, before the first in vivo measurement, the white reference needle is connected to the spectrometers to obtain a white reference. Thereafter, the white reference needle is detached and the measurement needle is attached to the spectrometers. Switching these needles might induce intensity differences between patients. These intensity differences are again not tissue but are related to differences in the measurement set-up.

Normalization can be performed in several ways, using the AUC, at $800 \mathrm{~nm}$, using SNV normalization. The later will remove scattering information from the measured spectra, potentially removing part of the discriminating information, as might have been the case in the diffuse reflection HSI dataset in Chapter 7. Therefore, preferably normalization using the intensity at $800 \mathrm{~nm}$ or using the AUC should be used, as concluded in Chapter 7. 
To prevent the need for normalization, the white reference procedure should be adjusted. Most importantly the switch between needles after the white reference should be avoided. This could be done by providing a white reference of which at least the needle holder can be sterilized. The white reference is performed on $99 \%$ Spectralon, which is a calibrated material. Sterilization of Spectralon is not recommended, because this might change the optical properties. Because the white reference is performed at a distance to the Spectralon surface it should be sufficient to sterilize only the holder and not the Spectralon. Second, if disposable needle are used, care should be taken to use only needles with exactly the same properties to prevent differences in measured spectra.

\section{Feature extraction}

In previous research done in our group, the obtained spectra were often fitted using an analytical model based on optical diffusion theory [13-15]. This algorithm can be seen as a feature extraction method, in which biological parameters are obtained based on prior knowledge of light transport and the spectral properties of the tissue constituents [16]. The optical diffusion theory on which this model is based, makes several assumptions. First, it assumes light has undergone several scattering events within the tissue before it is detected. This means that fibers should be placed at a distance within which several scattering events should occur. Second, it assumes the tissue to be homogeneous [17]. However, colorectal wall consists of several layers, making this assumption invalid. Therefore, in this thesis the use of the analytical model is avoided and other feature extraction methods are used.

In Chapter $\mathbf{2}$ and 3, spectral bands are used to reduce the number of feature used for the classification. The spectra are divided into spectral bands based on intensity values. The starting points of the bands are chosen randomly, which might result in slightly different bands each time the bands are determined. Therefore, the reproducibility of the results is slightly less, when compared to a division made based on non-random start points. On the other hand, by using these random start points, the chances of overfitting the classification are reduced because no prior knowledge is used. The division into spectral bands is only based on the intensity values of the spectra and does not use any prior knowledge about tissue constituents or spectral characteristics which are tissue specific. Therefore, the use spectral bands reduces the chance of overfitting but is not a robust technique for feature extraction. 
In Chapter 7, two different feature extraction methods are tested, one of which is based on prior knowledge (the shape-based features) and one which is not (peak data). It is concluded that the use of prior knowledge in feature extraction has an advantage over feature extraction that does not include any prior knowledge. It was even found in Chapter $\mathbf{7}$ that the use of the fit parameters is the preferred feature extraction method if the fiber distance is large enough to meet the assumption of the diffusion theory. The use of the fit algorithm should however be avoided in cases in which the assumptions made in the model are not valid such as when the fiber distance is too small or in the use of diffuse reflection HSI where too much glare is present in the signal. Because this is the case in colorectal cancer, it is recommended to use a different knowledge based feature extraction like the shape-based features presented in Chapter 7.

\section{Classifier}

In Chapter 7, a comparison was made between different classifiers. A major issue in classifier selection is the chance of overfitting. The chances of overfitting increase when the number of features used for the classification is close to the number of data samples included in the study. Especially for medical data, it is often hard to include a large number of patients in a dataset. When entire spectra are used for the classification as is done in Chapter 5, 1200 features (intensity values for each wavelength) are used for the classification. If a decision tree classifier is used to classify data from just 32 patients, overfitting of the classifier will most likely happen. In Chapter 7, it was found that SVM and neural networks (NN) are least sensitive to overfitting and therefore are the best options to use in classification of spectral data. For diffuse reflection $\mathrm{HSI}$, the chances of overfitting are less because of the large number of measurements, i.e. pixels obtained in one image. Therefore, also classifiers that are more sensitive to overfitting could be used for this type of data.

\subsection{Outlook}

In this thesis it has been shown that both fiberoptic DRS and diffuse reflection HSI are capable of distinguishing tumor tissue from fat and healthy colorectal wall, for fiberoptic DRS this was even shown in an in vivo setting. With the improvements in neoadjuvant therapies for patients with rectal cancer, the need for intraoperative 
tissue classification seems to diminish. Furthermore, the majority of colorectal tumors will be detected in an earlier stage with the introduction of the recently introduced population screening. However, there will still be advanced stage tumors that need to be surgically removed. Especially in pre-treated patients it will be hard for the surgeon to distinguish vital tumor tissue and fibrotic tissue during surgery. In these patients optical tissue characterization will be of added value. There is however, one major prerequisite, which is the ability of optical techniques to make the distinction between tumor and fibrosis. As long as that has not been proven, further steps should be taken with caution. However, if it has been proven that it is possible to distinguish tumor tissue from fibrosis, fiber optics DRS and diffuse reflection HSI will be of major added value to the surgeon. Fiberoptic DRS could be incorporated into a surgical instrument, giving the surgeon a chance to look a few millimeters ahead. Diffuse reflection HSI could be incorporated into a laparoscopic camera, providing the surgeon with a view in which the tumor is highlighted by the information provided by the hyperspectral camera. The development of such a laparoscopic camera could be supported by the data already obtained with fiberoptic DRS. The data from the fiberoptic DRS could provide discriminating wavelengths which could be used in the development. As data from diffuse reflectance HSI is slightly different compared to fiberoptic DRS, classification algorithms can not be used interchangeably between the two techniques. However, data from fiberoptic DRS could point the development of diffuse reflectance $\mathrm{HSI}$ in the right direction. 


\section{References}

[1] G. C. Langhout et al., "Differentiation of healthy and malignant tissue in colon cancer patients using optical spectroscopy: A tool for image-guided surgery," Lasers in Surgery and Medicine, vol. 47, no. 7, pp. 559-565, 2015.

[2] R. M. Schols, P. Dunias, F. P. Wieringa, and L. P. S. Stassen, "Multispectral characterization of tissues encountered during laparoscopic colorectal surgery," Medical Engineering and Physics, vol. 35, no. 7, pp. 1044-1050, 2013.

[3] L.-H. Li et al., "Monitoring neoadjuvant therapy responses in rectal cancer using multimodal nonlinear optical microscopy," Oncotarget, vol. 8, pp. 107323-107333, nov 2017.

[4] M. Gutowski et al., "SGM-101: An innovative near-infrared dye-antibody conjugate that targets CEA for fluorescence-guided surgery," Surgical Oncology, vol. 26, pp. 153-162, jun 2017.

[5] I. D. Nagtegaal and P. Quirke, "What Is the Role for the Circumferential Margin in the Modern Treatment of Rectal Cancer?," Journal of Clinical Oncology, vol. 26, no. 2, pp. 303-312, 2008.

[6] C. Simillis et al., "A systematic review to assess resection margin status after abdominoperineal excision and pelvic exenteration for rectal cancer," Annals of surgery, vol. 265, no. 2, pp. 291299, 2017.

[7] A. J. Gomes and V. Backman, "Algorithm for automated selection of application-specific fiberoptic reflectance probes," Journal of Biomedical Optics, vol. 18, p. 27012, feb 2013.

[8] R. Hennessy et al., "Effect of probe geometry and optical properties on the sampling depth for diffuse reflectance spectroscopy," Journal of biomedical optics, vol. 19, p. 107002, oct 2014.

[9] J. R. Parfitt and D. K. Driman, "The total mesorectal excision specimen for rectal cancer: a review of its pathological assessment," Journal of clinical pathology, vol. 60, pp. 849-855, aug 2007.

[10] G. Lu and B. Fei, "Medical hyperspectral imaging: a review," Journal of Biomedical Optics, vol. 19, p. 10901, jan 2014.

[11] S. Grusche, "Basic slit spectroscope reveals three-dimensional scenes through diagonal slices of hyperspectral cubes," Appl. Opt., vol. 53, pp. 4594-4603, jul 2014.

[12] W. R. Johnson et al., "Snapshot hyperspectral imaging in ophthalmology," Journal of Biomedical Optics, vol. 12, pp. 1-7, jan 2007.

[13] D. J. Evers et al., "Diffuse reflectance spectroscopy: a new guidance tool for improvement of biopsy procedures in lung malignancies," Clinical lung cancer, vol. 13, no. 6, pp. 424-431, 2012.

[14] L. L. Boer et al., "Fat/water ratios measured with diffuse reflectance spectroscopy to detect breast tumor boundaries," Breast Cancer Research and Treatment, vol. 152, no. 3, pp. 509-518, 2015.

[15] J. W. Spliethoff et al., "Real-time in vivo tissue characterization with diffuse reflectance spectroscopy during transthoracic lung biopsy: a clinical feasibility study," Clinical Cancer Research, vol. 22, no. 2, pp. 357-365, 2015. 
[16] T. M. Bydlon et al., "Chromophore based analyses of steady-state diffuse reflectance spectroscopy: current status and perspectives for clinical adoption," Journal of Biophotonics, vol. 8 , pp. 9-24, jan 2015.

[17] T. J. Farrell, M. S. Patterson, and B. Wilson, "A diffusion theory model of spatially resolved, steady-state diffuse reflectance for the noninvasive determination of tissue optical properties in vivo," Medical Physics, vol. 19, no. 4, pp. 879-888, 1992. 
Appendix A

Appendix 


\section{English summary}

The standard treatment in patients with colorectal cancer is surgery. For locally advanced rectal cancer this is more often combined with neoadjuvant (chemo)radiotherapy. During colorectal cancer surgery a balance should be found between complete removal of the tumor and sparing as much healthy tissue as possible to prevent complications. A technique that can provide real-time tissue classification during surgery might be of great benefit for the surgeon to prevent positive resection margins and complications due to too extensive surgery. In this thesis fiberoptic diffuse reflectance spectroscopy (DRS) and diffuse reflection hyperspectral imaging $(\mathrm{HSI})$ are examined as real-time tissue classification techniques that can ultimately be used during colorectal cancer surgery. In both techniques the interaction between tissue and light is examined. Providing information on tissue constituents and structure. Based on this information healthy tissue could potentially be discriminated from tumor tissue in colorectal cancer.

\section{Chapter 2}

In Chapter 2, fiberoptic DRS is first examined on tissue samples obtained at the pathology department after resection. At the pathology department three tissue samples were obtained - fat, healthy colorectal wall and tumor tissue - which were all placed in a pathology cassette. The samples remained in the cassette during the measurements and during further processing at the pathology department. Images were taken of each measurement location, to eventually register each measurement location to the histological images obtained of each tissue sample. The fiberoptic DRS spectra were divided into spectral bands based on intensity values. The intensities of these spectral bands were used for classification of the spectra using a quadratic classifier and a support vector machine (SVM). The quadratic classifies was used to distinguish fat from healthy colorectal wall and tumor tissue. The SVM was trained to separate healthy colorectal wall from tumor tissue. To train and test both classifiers, the dataset was randomly divided into a train (80\%) and test (20\%) set. The training and testing of the classifiers was repeated ten times, using different randomly selected train and test datasets.

In total 38 patients were included in this study. Of these 38 patient, 36 had colorectal cancer and 2 had an adenoma. The quadratic classifier obtained a train accuracy of 1.00 . The train accuracy of the SVM was 0.92 . When combining the 
two classifiers and applying this pipeline to the test dataset, a mean accuracy of $0.95( \pm 0.03)$ over all tissue types and the ten repetitions was obtained. The best accuracy was obtained for fat $(1.00 \pm 0.00)$, followed by healthy colorectal wall $(0.93 \pm 0.05)$ and tumor $(0.92 \pm 0.09)$. When measurement locations which were located at the border between two tissue types were classified, the classification of $80 \%$ of the locations was in accordance with the most prominent tissue type present at this location.

\section{Chapter 3}

Similar tissue samples that were used in Chapter 2, were imaged using two hyperspectral cameras in Chapter 3 . In this chapter registration of the pathology classification was done for the entire tissue sample. After registration, the diffuse reflection hyperspectral images were normalized using the standard normal variate (SNV) normalization. Thereafter, the dataset was randomly divided into a train and test set. The train dataset contained $75 \%$ of the patients and the test dataset contained the remaining $25 \%$. Feature reduction was again done using spectral bands, based on the intensity values of the spectra. Using these features, two classifiers were trained. A quadratic classifier to classify fat and a SVM to separate tumor tissue from healthy colorectal wall.

In this study 54 patients were included. The tissue samples of 32 patients were imaged with both hyperspectral cameras, the remaining 22 patients were only imaged with the hyperspectral camera covering the near-infrared wavelength range. When data obtained with both hyperspectral cameras were used for classification an average accuracy over all tissue types of $0.88( \pm 0.13)$ was obtained. When data of just one of the cameras was used for classification the average accuracy decreased to 0.67 and 0.83 for the camera in the visual wavelength range and near-infrared wavelength range, respectively. From this it was concluded that HSI could be used in tissue classification during colorectal cancer surgery, but that the combination of both cameras seems necessary for the most optimal classification.

\section{Chapter 4}

In locally advanced rectal cancer, patients often receive neoadjuvant (chemo)radiotherapy before surgery. The neoadjuvant radiotherapy causes fibrosis around the tumor area. Fibrosis is classified by the pathologist as healthy tissue and can thus remain in the patient during surgery. However, due to similar visual and tac- 
tile feedback for fibrosis and tumor it is often hard for the surgeon to discriminate fibrosis from tumor. Here, again a real-time tissue classification technology could be of great benefit to the surgeon. Therefore, in Chapter 4 a classification is trained and tested on the discrimination of fibrosis from tumor.

In this study fiberoptic DRS measurement were performed on the entire specimen of 38 patients with rectal cancer. Due to the limited number of pure tumor measurements obtained in these 38 patients ( 5 measurements with tumor at the surface) tumor measurements from Chapter 2 were used in a first classification. In this classification a SVM was trained and tested on pure fibrosis and pure tumor measurements. Training and testing of the classifier was performed using a ten-fold cross-validation, which was repeated ten times. The first classification resulted in a mean test accuracy of 0.88 ( \pm 0.02 ), a mean sensitivity of 0.91 ( \pm $0.01)$, a mean specificity of $0.86( \pm 0.03)$ and a mean Matthews correlation coefficient (MCC) of $0.76( \pm 0.03)$. Thereafter, a second SVM was trained and tested on data that was obtained only in the current study, resulting in almost no pure tumor measurements. This classification resulted in a mean accuracy of 0.61 ( \pm $0.05)$, a mean sensitivity of $0.51( \pm 0.10)$, a mean specificity of $0.66( \pm 0.11)$ and a mean MCC of $0.17( \pm 0.08)$.

The decrease in all performance measures could have several explanations. First of all, in the first classification, data of two different studies were combined. Even though, the measurement set-up and measurement probe used in both studies were the same, differences between the datasets could have been present due to the different nature of the tissues. In the study reported in Chapter 2, tissue slices were used to perform measurements on, whereas in the current study entire rectum specimen were used. This different might have caused differences between the tumor measurements and fibrosis measurements. Another explanation is the limited number of pure tumor measurement that were measured in the current study. Only 5 measurements were performed on locations at which tumor was found at the surface. Creating a classification based on such limited data is hard. In the spectral analysis performed in this chapter it was shown that there is a spectral difference between pure tumor and pure fibrosis measured in the current study. However, it is hard to discriminate tumor with a layer of fibrosis on top from pure fibrosis. 


\section{Chapter 5}

In Chapter 5, fiberoptic DRS measurements were performed during surgery. Measurements were performed by the surgeon on fat, healthy colorectal wall and on a location close to the tumor. All measurement locations were marked by the surgeon for pathology verification. The measurements were processed and classified after surgery. Before classification the spectra were normalized at $800 \mathrm{~nm}$. Thereafter, two SVMs were trained and tested using a ten-fold cross validation. The first SVM was used to discriminate fat from healthy colorectal wall and tumor. The second SVM was used to discriminate healthy colorectal wall from tumor. Besides the classification, the influence of the depth of the tumor in the measurement volume on the classification was analyzed. The analysis was performed by increasing the depth of the tumor, at which a measurement location was classified as tumor. Furthermore, the classification results were compared to the clinical judgement of the surgeon to show the added value of this technology.

Measurements of 32 patients were included in the analysis. Classification of fat was done with a mean MCC of 0.83 , for healthy colorectal wall the mean MCC was 0.77 and for tumor the mean MCC was 0.73 . In the depth analysis it was shown that the best accuracy and MCC values were obtained when a measurement was classified as tumor, if tumor was present up to a maximum depth equal to the fiber distance. With increasing depths of the tumor, the accuracy and MCC values showed a big decrease. Which was expected because if tumor is only present at larger depths, the amount of tumor present in the measured volume is too small to detect using fiberoptic DRS. With decreasing depths, a decrease in accuracy and MCC values was shown as well. This was not expected, but could be explained by the limited number of measurement locations in which tumor was present this superficial.

Finally, the outcome of the classification was compared to the clinical judgement. For this analysis only measurement were taken into account of which the surgeon indicated not to be sure whether tumor was present, 54 out of 270 locations. No false negative classification were allowed in this analysis. It was found that with a threshold for the classification allowing no false negative predictions, $25 \%$ of the healthy locations were falsely classified as tumor. For the surgeon $69 \%$ of the healthy locations were falsely classified as tumor. This shows the potential added value of fiberoptic DRS in surgery. 


\section{Chapter 6}

In Chapter 6, a comparison is made between measurements obtained in an in vivo setting and measurements obtained ex vivo. For new technologies it is common practice to first perform ex vivo measurements, which are thereafter repeated in vivo. The ex vivo measurements are used to prove a concept, but because in vivo optical properties might change, measurements are always repeated in vivo. In this chapter it was examined whether ex vivo measurements differ significantly from in vivo measurements and if ex vivo measurements can be used to train a classification that can be used on in vivo measurements.

For the analysis a cross-correlation of the data was assumed because of the correlation of measurements performed within one patient and the correlation of measurement either performed in vivo or ex vivo. The analysis was performed on parameters obtained using an analytical fit model which is based on optical diffusion theory. It was found that only the diameter of the blood vessels and the scale factor did not differ significantly between in vivo and ex vivo for all tissue types.

Even though, almost all parameters differed significantly between in vivo and ex vivo, there was no significant difference between the classification outcomes of a classifier trained and tested on in vivo data and a classifier trained on ex vivo data and tested on in vivo data. Based on forward feature selection four parameters were selected to create a classification with a reduced number of parameters. The four selected parameters were the blood volume fraction, the saturation of the blood, the volume fraction of water and fat and the volume fraction of fat from water plus fat. With these selected parameters similar results were obtained, compared to the classification using all parameters. This shows that is it possible in colorectal cancer to include ex vivo measurements in the training of a classification, that is used for in vivo measurement classification.

\section{Chapter 7}

In this thesis several different analysis techniques were used. In the final chapter it is examined which analysis technique performs best for the classification of healthy colorectal wall versus tumor. In this chapter, normalization techniques, feature extraction techniques, and different classifiers are examined in nine different datasets.

It was found that overfitting is a problem in complex classifiers like k-nearest 
neighbor (kNN), linear discriminant analysis (LDA) and decision tree classifiers. Especially when the entire spectrum (1151 wavelengths) is used for classification, overfitting is present from these three classifiers. If feature reduction techniques are applied, the chances of overfitting decrease for both LDA and kNN. For decision tree classification, even with feature reduction there is a large chance of overfitting. Overall SVM and neural network (NN) classifiers showed the best results with the least chance of overfitting.

Normalization overall did not seem to improve classification results. However, it was found that in the fiberoptic DRS datasets, in which disposable needles were used, normalization did improve the classification outcome. This might have to do with small differences between the disposable needles used in for these two dataset. Moreover, between the white reference and the actual measurements, a switch needed to be made between the white reference needle and the measurement needle. This switch might have introduced intensity differences which were not patient or tissue specific. To overcome these intensity differences, normalization might be a solution. If normalization is used, it is recommended to use normalization at $800 \mathrm{~nm}$, instead of normalization using the area under the curve or SNV normalization.

Comparing different feature extraction techniques to the use of the entire spectrum, it was found that it is most useful to use features that describe the shape of the spectrum as good as possible. The parameters obtained with the analytical model based on optical diffusion theory were found to be the best parameters used for classification. However, these parameters cannot always be used due to assumptions made in the model on fiber distance and the assumption that illumination was done with a collimated beam, excluding diffuse reflection HSI. If fit parameters could not be used, shape-based features were the best feature extraction method. It was concluded that the optimal classification of healthy colorectal wall and tumor is done using a SVM or NN, using fit parameters or shape-based features, without normalization. 


\section{Nederlandse samenvatting (Dutch summary)}

Chirurgie is de standard behandeling voor patiënten met colorectaal tumoren. Voor patiënten met een lokaal vergevorderd stadium rectum tumor wordt chirurgie vaak gecombineerd met (chemo) radiotherapie. Tijdens chirurgie, voor colorectaal tumoren, moet er een balans gevonden worden tussen het compleet verwijderen van de tumor en het sparen voor zo veel mogelijk gezond weefsel om complicaties te voorkomen. Een techniek die real-time weefsel kan classificeren in gezond en tumor weefsel zou van grote meerwaarde kunnen zijn voor de chirurg om positieve resectie marge en complicaties te voorkomen.

In deze thesis werden optische fiber diffusie reflectie spectroscopie (DRS) en diffusie reflectie hyperspectrale imaging $(\mathrm{HSI})$ onderzocht als real-time weefsel classificatie technieken, met als doel deze toe te passen tijdens colorectaal chirurgie. In beide technieken wordt gekeken naar de interactie van licht met weefsel. Hierbij wordt informatie verkregen over de structuur en bestandsdelen van het weefsel. Gebaseerd op deze informatie wordt geprobeerd onderscheidt te maken tussen gezond weefsel en tumor weefsel in colorectaal tumoren.

\section{Hoofdstuk 2}

In Hoofdstuk 2 werd optische fiber DRS gebruikt voor het meten van weefselmonsters. Op de pathologie afdeling werden drie weefsel types verkregen - vet, gezonde darmwand en tumor - die in een pathologie cassette geplaatst werden. De weefsels bleven in deze cassette tijdens de metingen en daarna tijdens verwerking op de pathologie afdeling. Fiber optische DRS werd gebruikt voor het doen van metingen en van elke locatie werd een foto gemaakt zodat elke meet locatie uiteindelijk geregistreerd kon worden aan de verkregen pathologie.

De optische fiber DRS spectra verkregen tijdens de metingen werden opgedeeld in spectrale banden gebaseerd op intensiteit. De intensiteit van deze spectrale banden werd gebruikt voor de classificatie van de spectra. Voor deze classificatie werd gebruikt gemaakt van een kwadratische classificatie en een support vector machine (SVM). De kwadratische classificatie werd getraind om vet van gezonde darmwand en tumor te scheiden, de SVM om gezonde darmwand van tumor te onderscheiden. Om beide classificaties te trainen en testen werd de dataset willekeurig verdeeld in een train $(80 \%)$ en test $(20 \%)$ dataset. Het trainen en testen van de classificatie werd tien keer herhaald, waarbij de train en test dataset elke keer willekeurig wordt geselecteerd. 
In totaal werden weefsels van 38 patiënten gemeten. Van deze 38 patiënten hadden 36 patiënten een colorectaal tumor en 2 een adenoom. De train nauwkeurigheid voor de classificatie van vet was 1.00. De train nauwkeurigheid voor de SVM, om gezonde darmwand van tumor te onderscheiden, was 0.92 . Als beide classificaties achter elkaar werden gezet en toegepast op de test dataset werd er een gemiddelde nauwkeurigheid van $0.95( \pm 0.03)$ over alle weefsel types en alle tien herhalingen verkregen. De beste nauwkeurigheid werd behaald voor vet (1.00 \pm $0.00)$, gevolgd door gezonde darmwand $(0.93 \pm 0.05)$ en tumor $(0.92 \pm 0.09)$. Locaties die zich op de grens tussen twee weefsel types bevonden, werden in $80 \%$ van de gevallen geclassificeerd als het weefsel type dat het meest aanwezig was in het meetvolume.

\section{Hoofdstuk 3}

Vergelijkbare weefsels als die in Hoofdstuk 2 werden gemeten, werden ook onder twee hyperspectrale camera's gelegd, de resultaten hiervan worden in Hoofdstuk 3 besproken. In dit hoofdstuk werd de registratie tussen de pathologie en de hyperspectrale afbeeldingen gedaan voor het gehele stuk weefsel. Hierdoor was voor iedere pixel in de diffusie reflectie hyperspectrale afbeelding een pathologische classificatie beschikbaar. De diffusie reflectie hyperspectrale afbeeldingen werden genormaliseerd doormiddel van een standard normal variat (SNV) normalisatie. Vervolgens werd de dataset willekeurig verdeeld in een train en test dataset, waarbij de train dataset $75 \%$ van de patiënten bevatte en de test dataset $25 \%$. Het aantal features dat gebruikt werd voor de classificatie werd gereduceerd met behulp van spectrale banden. Deze banden werden bepaald op basis van de intensiteit van de spectra en gebruikt voor het trainen en testen van twee classificaties. Eerst werd een kwadratische classificatie getraind voor het onderscheiden van vet en vervolgens een SVM voor het classificeren van gezonde darmwand en tumor.

In deze studie werden 54 patiënten geïncludeerd. Van deze patiënten werden er van 32 afbeeldingen gemaakt met beide hyperspectrale camera's. Van de overige 22 patiënten werden alleen afbeeldingen gemaakt met de hyperspectrale camera die signaal opvangt in het nabij infrarode golflengte gebied. Als data van beide camera's werd gebruikt voor classificatie, resulteerde dat in een nauwkeurigheid van 0.88 ( \pm 0.13$)$ gemiddeld over alle weefseltypes. Als enkel data van een van beide camera's werd gebruikt voor classificatie, ging de nauwkeurigheid omlaag naar 0.67 en 0.83 voor respectievelijk data van de camera in het visuele golflengte 
gebied en data van de camera in het nabij infrarode golflengte gebied. Hieruit kon worden geconcludeerd dat diffuse reflectie HSI gebruikt kan worden voor weefsel classificatie in colorectaal tumoren en dat voor optimaal resultaat beide golflengte gebieden moesten worden gebruikt.

\section{Hoofdstuk 4}

Patiënten met lokaal uitgebreide rectaal tumoren, krijgen vaak een combinatie van chirurgie met neoadjuvante (chemo)radiotherapie. De neoadjuvante radiotherapie zorgt er voor dat er fibrose ontstaat rond de tumor. Omdat fibrose door de patholoog niet wordt gezien als kwaadaardig weefsel mag dit tijdens de operatie achter blijven in de patiënt. Echter, hebben fibrose en tumor dezelfde visuele en tactiele feedback, waardoor het voor een chirurg moeilijk is om beide van elkaar te onderscheiden. Het gebruik van real-time weefsel classificatie technologie zou hier van grote meerwaarde kunnen zijn voor de chirurg. Daarom was het onderzoek in Hoofdstuk 4 gefocust op het trainen en testen van een classificatie voor het onderscheid van tumor en fibrose.

In deze studie werden optische fiber DRS metingen uitgevoerd op hele preparten van 38 patiënten met rectum tumoren. Doordat er in deze 38 patiënten maar een zeer klein aantal metingen op pure tumor was gedaan (5 metingen waarbij tumor aan het oppervlak zat), werd er besloten om tumor metingen uit Hoofdstuk 2 te gebruiken voor een eerste classificatie. In deze eerste classificatie werd een SVM getraind op pure tumor en pure fibrose metingen. Het trainen en testen van de SVM werd gedaan met behulp van een tienvoudige cross-validatie, die tien keer is herhaald. Deze eerste classificatie resulteerde in een gemiddelde nauwkeurigheid van 0.88 ( \pm 0.02$)$, een gemiddelde sensitiviteit van 0.91 ( \pm 0.01 ), een gemiddelde specificiteit van $0.86( \pm 0.03)$ en een gemiddelde Matthews Correlatie Coëfficiënt $(\mathrm{MCC})$ van $0.76( \pm 0.03)$. Hierna werd een tweede SVM getraind en getest waarbij enkel gebruik werd gemaakt van data verkregen binnen de huidige studie, waardoor er bijna geen pure tumor metingen aanwezig waren in de dataset. Deze classificatie resulteerde in een gemiddelde nauwkeurigheid van $0.61( \pm 0.05)$, een gemiddelde sensitiviteit van $0.51( \pm 0.10)$, een gemiddelde specificiteit van $0.66( \pm 0.11)$ en een gemiddelde MCC van $0.17( \pm 0.08)$.

De afname die werd gezien in alle prestatie maten kan verschillende verklaringen hebben. Allereerst werd de eerste classificatie gedaan op een combinatie van twee verschillende datasets. Ondanks dat de meetopstelling en de naald waarmee de metingen werden uitgevoerd hetzelfde waren in beide studies, zouden 
er verschillen geweest kunnen zijn in beide datasets door het verschil in de weefsels die zijn gemeten. In de studie van Hoofdstuk 2 werden weefselmonsters gebruikt om op te meten, terwijl er in de huidige studie werd gemeten op volledige preparaten. Dit verschil zou gezorgd kunnen hebben voor een verschil tussen de datasets op basis waarvan geclassificeerd is. Een andere verklaring zou kunnen zijn dat er maar een klein aantal pure tumor metingen werd gemeten in de huidige studie. Er werden maar 5 metingen gedaan waarbij tumor aan het oppervlak aanwezig was. Het is moeilijk om met een dergelijk kleine dataset een goede classificatie te maken. In de spectrale analyse van dit hoofdstuk was te zien dat er weldegelijk spectrale verschillen waren tussen de spectra van pure tumor en pure fibrose. Het was echter moeilijk om tumor met daarop een laag fibrose te onderscheiden van pure fibrose.

\section{Hoofdstuk 5}

In Hoofdstuk 5 werden optische fiber DRS metingen gedaan tijdens de operatie. De metingen werden uitgevoerd door de chirurg op vet, gezonde darmwand en op een locatie dicht bij de tumor. Alle metingen werden gemarkeerd door de chirurg voor verificatie bij de pathologie. Classificatie van de metingen werd gedaan na afloop van de operatie. Voor de classificatie werden alle spectra genormaliseerd op $800 \mathrm{~nm}$. Vervolgens werden er twee SVMs getraind en getest met behulp van een tienvoudige cross-validatie. De eerste SVM werd gebruikt om vet te onderscheiden van gezonde darmwand en tumor. De tweede SVM werd gebruikt om gezonde darmwand te onderscheiden van tumor. De invloed van de diepte van de tumor, ten opzichte van het meetoppervlak, op het resultaat van de classificatie werd ook geanalyseerd. Dit werd geanalyseerd door metingen met oplopende diepte van tumor te labelen als tumor. Uiteindelijke werden de classificatie resultaten vergeleken met het klinisch oordeel van de chirurg om zo de meerwaarde van de techniek te laten zien.

Metingen van 32 patiënten werden geïncludeerd in de analyse. Classificatie van vet kon worden gedaan met een gemiddelde MCC van 0.83 , voor gezonde darmwand was de gemiddelde MCC 0.77 en voor tumor 0.73 . De analyse van de diepte van de tumor liet zien dat als tumor voor het eerst word gedetecteerd op een diepte gelijk aan de fiber afstand, de beste MCC en nauwkeurigheid werd behaald. Als metingen met tumor op grotere diepte, werden geïncludeerd als tumor was een afname te zien in de nauwkeurigheid en MCC waardes. Dit werd verwacht, omdat als tumor enkel aanwezig is op grotere diepte, het aandeel tu- 
mor in de het gemeten volume te klein is om gedetecteerd te worden met behulp van de optische fiber DRS. Als de maximale diepte van tumor minder werd dan de fiber afstand, was ook een afname in MCC en nauwkeurigheid te zien. Dit was niet verwacht maar kon verklaard worden door het kleine aantal metingen met tumor zo dicht bij het oppervlak.

Ten slotte werden de uitkomsten van de classificatie vergeleken met de klinische beoordeling van de chirurg. Voor deze analyse werden alleen de metingen meegenomen waarvan de chirurg aangaf niet zeker te zijn of deze tumor bevatte, 54 van de 270 locaties. Voor deze analyse werden geen vals negatieve classificaties toegestaan om positieve resectie marges te voorkomen. Als de classificatie grens zo werd aangepast dat er geen valse negatieve classificaties waren, werd $25 \%$ van de locaties foutief geclassificeerd als tumor. Bij de klinische beoordeling door de chirurg werd $69 \%$ van de locaties foutief geclassificeerd als tumor. Dit liet de potentiele toegevoegde waarde zien van het gebruik van optische fiber DRS tijdens chirurgie.

\section{Hoofdstuk 6}

In Hoofdstuk 6 werd er een vergelijk gemaakt tussen metingen gedaan in vivo en metingen die ex vivo waren gedaan. Voor nieuwe technologieën is de normale procedure om eerst ex vivo metingen uit te voeren en deze vervolgens in vivo te herhalen. De ex vivo metingen worden gedaan om het principe te bewijzen, maar omdat optische eigenschappen van het weefsel mogelijk verschillen tussen de in vivo en ex vivo metingen, moeten de metingen ook in vivo gedaan worden. In dit hoofdstuk werd onderzocht of ex vivo metingen significant verschillen van in vivo metingen en of het mogelijk was om een classificatie te trainen op ex vivo data en die vervolgens te gebruiken om in vivo metingen correct te classificeren.

Voor de analyse werd uit gegaan van cross-correlatie in de data vanwege correlatie van metingen gedaan in één patiënt, de correlatie binnen verschillende wisseltypes en de correlatie binnen metingen die in vivo dan wel ex vivo gedaan waren. De analyse werd uitgevoerd met de parameters die werden verkregen uit een analytisch fit model dat is gebaseerd op optische diffusie theorie. Uit de analyse kwam dat enkel de diameter van de bloedvaten en de schaal factor niet significant verschillen tussen de in vivo en ex vivo metingen van alle weefsel types.

Ondanks dat bijna alle parameters significant verschilden tussen de in vivo en ex vivo metingen werden er geen significante verschillen gevonden tussen de clas- 
sificaties als de classificatie werd getraind en getest op in vivo data of als de classificatie werd getraind op ex vivo data en getest op in vivo data. Met behulp van forward feature selectie werden er vier parameters geselecteerd voor een classificatie met een gereduceerd aantal parameters. De vier geselecteerde parameters waren de fractie bloed volume, de saturatie van het bloed, de volume fractie water plus vet, en de volume fractie vet van water plus vet. Met deze vier parameters werden vergelijkbare resultaten behaald voor de classificatie, in vergelijking met de classificaties waarvoor alle parameters werden gebruikt. Dit liet zien dat het mogelijk was in colorectale tumoren om ex vivo metingen te gebruiken voor het trainen van een classificatie die vervolgens in vivo metingen classificeert.

\section{Hoofdstuk 7}

In deze thesis werden verschillende analyse technieken gebruikt. In het laatste hoofdstuk werd onderzocht welke analyse techniek het best werkt voor de classificatie van gezonde darmwand versus tumor. In dit hoofdstuk werden normalisatie technieken, feature extractie technieken en verschillende classificatie methodes ongezocht waarbij gebruikt werd gemaakt van negen verschillende datasets.

Overfitting van de classificatie methode bleek vooral een probleem te zijn in complexe classificatie methodes zoals k-nearest neighbor (kNN), linear discriminant analysis (LDA) en beslissingsboom classificaties. Zeker als het volledige spectrum werd gebruikt voor de classificatie werd overfitting gezien in de drie bovengenoemde classificatie methodes. Als feature extractie werd gebruikt, om het aantal features dat gebruikt werd voor de classificatie te reduceren, werd er een afname gezien in de kans op overfitting voor kNN en LDA. De beslissingsboom classificatie bleef zelfs met feature extractie overfitten. Over het algemeen gaven SVM en neural network (NN) classificaties de beste resultaten als het ging om de kans op overfitting.

Normalisatie van de data leek over het algemeen gezien geen verbetering van de classificatie te geven. Echter, in de optische fiber DRS datasets waarbij gebruik werd gemaakt van wegwerp naalden verbeterde normalisatie de classificatie resultaten wel. Dit zou kunnen komen door kleine verschillen tussen de naalden die werden gebruikt tijdens de metingen in deze twee datasets. Verder, moest er bij deze datasets gewisseld worden tussen de referentie naald en de meet naald. Deze wissel vond plaats tussen de wit referentie en de metingen op het weefsel. Door deze wissel zouden intensiteitsverschillen kunnen zijn ontstaan die niet patiënt of weefsel specifiek waren. Normalisatie van de metingen was een 
oplossing voor het overkomen van deze intensiteitsverschillen. Als normalisatie werd gebruikt, werd geadviseerd om te normaliseren op $800 \mathrm{~nm}$, in plaats van normalisatie die gebruik maakt van het oppervlak onder de curve of SNV normalisatie.

Als verschillende feature extractie methodes werden vergeleken met het gebruik van het volledige spectrum werd gevonden dat de beste classificatie werd gedaan met behulp van feature die de vorm van het spectrum zo goed mogelijk beschreven. De parameters die uit het analytisch fit model komen, wat gebaseerd is op optische diffusie theorie, waren de beste features om te gebruiken voor de classificatie. Deze parameters kunnen alleen niet altijd gebruikt worden door aannames die gedaan zijn in het model voor de minimale afstand tussen de fibers en de aanname dat de belichting gebeurd door een gecollimeerde licht straal, wat diffusie reflectie HSI uitsluit. Als de fit parameters niet konden worden gebruikt, waren de vorm gebaseerde features de beste feature extractie methode. Concluderend was de combinatie van fit parameters of vorm gebaseerde features, zonder normalisatie met een SVM of NN classificatie het beste voor de classificatie van gezonde darmwand versus tumor. 


\section{Acknowledgments}

Geachte prof. dr. Ruers, best Theo, hartelijk bedankt voor de vier jaar dat ik me heb mogen ontwikkelen. Ik heb in de vier jaar van mijn promotie vaak gedacht dat er geen toekomst meer zat in het onderzoek dat ik deed. Jij wist me op die momenten altijd te motiveren en een weg te vinden die niet dood liep. Hoewel het project wellicht niet altijd de uitdaging heeft geboden die we hadden gehoopt, heb ik er wel met plezier aan gewerkt en heb ik veel geleerd. Bedankt voor alle hulp en wijze inzichten.

Geachte prof. Ir. Sterenborg, best Dick, bedankt voor alle discussies die we samen hebben gehad. Ik heb denk ik vaak tegen dingen aangeschopt en ik was wellicht niet altijd even enthousiast in het begin. Maar ik heb veel geleerd tijdens mijn promotie en ik denk dat we nuttige discussies hebben gehad over mijn werk. Beste Koert, heel erg bedankt voor de vele gesprekken die we hebben gehad over mijn promotie en de voortgang hiervan. Je was altijd erg enthousiast en kwam vaak met nieuwe ideeën over wat we nog meer zouden kunnen onderzoeken. Hoewel ik hier niet altijd iets mee deed of kon doen, heeft het me wel vaak aan het denken gezet over de mogelijkheden die er nog waren voor vervolg onderzoek. Ook tijdens de vele operaties waarbij ik in vivo metingen zou kunnen doen, heb je vaak met me mee gedacht. Het was altijd fijn om iemand erbij te hebben die meer af wist van de metingen en wist wat ik nodig had. Je welwillendheid om te helpen waardeer ik erg.

Lieve Sandra, ik zou je bijna vergeten, maar zonder jou zou mijn promotie een stuk lastiger zijn geworden. De inzet die jij altijd hebt gestoken in het helpen met alle administratieve zaken heb ik erg bewonderd. Heel erg bedankt voor al je hulp hierbij. Ook bedankt voor alle korte gesprekjes die we tussendoor hebben gehad. Ik denk dat jij als geen ander wist en weet wat er omgaat binnen onze onderzoeksgroep. Bedankt voor al je hulp en steun tijdens mijn promotie en nu ook nog bij het afronden van alles.

Beste collega's, bedankt voor al jullie hulp, de gezellige gesprekken en leuke lunches die we hebben gehad. Ik vond het fijn dat ik van tijd tot tijd mijn ideeën bij jullie kon neer leggen, maar we hebben ook veel lol gehad. Esther, heel erg bedankt voor alle energie die je hebt gestopt in het fit houden van je collega's. Ik ga niet snel vergeten dat ik een tijdje best lang kon planken en best veel kon opdrukken. Helaas neemt dat ook weer snel af als je het een tijdje niet doet.

Dear Behdad, as promised an entire paragraph just for you. Thank you so much 
for all the help you gave me during the final phase of my PhD. Discussing my analyses and the comments I received on my papers helped me a lot and made me more confident of my research. I hope you had as much fun as I had being your roommate during the final months. I think your are a very good scientist! I learned a lot from you and hope you also learned a bit from me about the optical part. A least you know now that not everything is about the fat water ratio. But as long as you apply the pressure correct you don't have to tilt.

Lieve Susan, heel erg bedankt voor al je steun en toeverlaat tijdens mijn promotie. Je oneindige geduld bij alle metingen die we hebben gedaan heb ik erg gewaardeerd. Ik heb er nog even over nagedacht om te tellen hoeveel metingen we samen hebben gedaan, maar dat leek een onmogelijke opgave. Onze koffiemomentjes in de week zal ik nooit vergeten. We konden elkaar dan vaak goed motiveren om weer wat harder te werken, maar wel pas na minstens een half uur koffiedrinken. Ik kan denk ik ook nooit meer niet aan je denken als ik Ed Sheeran op de radio hoor. Oh en om op de metingen en getallen terug te komen; de RGBcode van de kleur van de hoofdstukpagina's is .9 6. 6 .

Lieve vrienden heel erg bedankt voor jullie steun en interesse. Ik kan me voorstellen dat jullie van tijd tot tijd gek werden van al mijn gezeur over het aantal patiënten dat ik nog moest meten en het aantal artikelen dat nog geaccepteerd moest worden voor ik klaar zou zijn. Gelukkig is het nu allemaal klaar en kan ik jullie ook weer wat meer aandacht geven. Chica's, ik ga niet herhalen wat er in de afgelopen boekjes heeft gestaan. Bedankt voor de leuke dagjes weg en jullie support tijdens mijn promotie. Marloes, Marjolein, Martine, Heleen, Susan en Elanie, jullie in het bijzonder bedankt voor alle etentjes waarin ik mijn hart bij jullie kon luchten. Jullie hebben wellicht nooit helemaal begrepen wat ik deed, maar jullie frisse blik en de troostende woorden hebben mij zeker geholpen dit tot een goed einde te brengen.

Lieve Marloes en Marjolein, ik vind het erg bijzonder dat jullie vandaag naast mij staan. Sinds het eerste jaar van TG hebben we veel samen gedaan en heb ik het idee dat jullie mij erg goed begrijpen en er altijd voor mij zijn. Marloes, jouw nuchterheid in tijden dat ik het niet meer zag zitten, heeft er voor gezorgd dat ik alles een beetje kon relativeren. Marjolein, jouw kennis van zaken in de wetenschappelijke wereld was erg waardevol voor mij. Je spoedcursus paperschrijven in de tijd dat ik mijn eerste paper aan het schrijven was heeft mij, en ik weet zeker ook Theo, een hoop tijd bespaard.

Lieve familie en schoonfamilie, heel erg bedankt voor jullie interesse en steun. 
Het moet vast af en toe moeilijk zijn geweest om bij te houden wat ik deed en wat er nog moest gebeuren voor het allemaal klaar zou zijn. Ik vond het altijd erg fijn om er met jullie over te kunnen praten, maar om ook af en toe gewoon lekker te ontspannen bij jullie. Lieve opa en oma, ik vind het erg bijzonder dat jullie dit nog mee kunnen maken. Ook jullie waren altijd geïnteresseerd in wat ik deed en hoe ver ik nou was, nu kan ik gelukkig echt zeggen; het is klaar!

Lieve papa en mama, bedankt dat jullie er altijd voor mij zijn. Jullie adviezen neem ik altijd erg serieus, hoewel ik er misschien niet altijd wat mee doe. Jullie zijn altijd grote voorbeelden voor mij geweest. Om te horen dat jullie trots zijn op mij, doet mij goed. Ik weet dat jullie altijd achter mij staan en me zullen helpen zoveel als jullie kunnen. Daar wil ik jullie heel erg voor bedanken! Ik heb ik de afgelopen vier jaar best wel wat momenten gehad waarin ik het niet meer zag zitten om mijn promotie af te maken. Jullie hadden dan altijd goede raad en wisten me er altijd van te overtuigen dat het wel goed zou komen, dank jullie wel hiervoor. Maike en Paul, jullie natuurlijk ook heel erg bedankt voor jullie interesse en hulp.

Lieve Erik, wat moet ik nou nog tegen jou zeggen. Heel erg bedankt dat je vier jaar lang elke dag hebt geluisterd naar mijn verhalen over darmen, nasi bakjes, licht en spectra. Bedankt dat ik boos bij je mocht worden, met je mocht vechten en vieren. Ik denk dat het veel heeft geholpen dat we allebei in hetzelfde schuitje zaten maar dat dat soms ook extra zwaar was. Ik ben trots op ons, hoe we het de afgelopen jaren samen hebben gedaan. Ik weet zeker dat jij over een paar maanden ook een dankwoord aan het typen bent, dat vast beter wordt dan dit dankwoord. 


\section{PhD portfolio}

\section{PHD PERIOD: 2015-2019}

PhD supervisors: prof.dr. T.J.M. Ruers, prof.dr. H.J.C.M. Sterenborg, dr. K.F.D. Kuhlmann

\section{PUBLICATIONS}

E.J.M. Baltussen, P. Snaebjornsson, S.G. Brouwer de Koning, H.J.C.M. Sterenborg, A.G.J. Aalbers, N.F.M. Kok, G.L. Beets, B.H.W. Hendriks, K.F.D. Kuhlmann, T.J.M. Ruers, "Diffuse reflectance spectroscopy as a tool for real-time tissue assessment during colorectal cancer surgery", Journal of Biomedical Optics, 2017, Volume 22 (10)

S.G. Brouwer de Koning, E.J.M. Baltussen, M.B. Karakullukcu, B. Dashtbozorg, L.A. Smit, R. Dirven, B.H.W. Hendriks, H.J.C.M. Sterenborg, T.J.M. Ruers, "Toward complete oral cavity cancer resection using a handheld diffuse reflectance spectroscopy probe", Journal of Biomedical Optics, 2018, Volume 23 (12)

E.J.M. Baltussen, E.N.D. Kok, S.G. Brouwer de Koning, J.Sanders, A.G.J. Aalbers, N.F.M. Kok, G.L. Beets, C.C. Flohil, S.C. Bruin, K.F.D. Kuhlmann, H.J.C.M. Sterenborg, T.J.M. Ruers, "Hyperspectral imaging for tissue classification, a way towards smart laparoscopic colorectal surgery", Journal of Biomedical Optics, 2019, Volume 24 (1)

E.J.M. Baltussen, S.G. Brouwer de Koning, J. Sanders, A.G.J. Aalbers, N.F.M. Kok, G.L. Beets, B.H.W. Hendriks, H.J.C.M. Sterenborg, K.F.D. Kuhlmann, T.J.M. Ruers, "Tissue diagnosis during colorectal cancer surgery using optical sensing: an in vivo study", Journal of Translational Medicine, 2019, Volume 17(1)

E.J.M. Baltussen, S.G. Brouwer de Koning, K.Jozwiak, H.J.C.M. Sterenborg, T.J.M. Ruers, "Comparing in vivo and ex vivo fiberoptic diffuse reflectance spectroscopy in colorectal cancer", Translational Biophotonics, 2019

E.J.M. Baltussen, H.J.C.M. Sterenborg, T.J.M. Ruers, B. Dashtbozorg, "Optimizing tissue classification algorithms in colorectal cancer using diffuse reflectance", Biomedical Optics Express, 2019, Volume 10 (12) 
E.J.M. Baltussen, S.G. Brouwer de Koning, J. Sanders, A.G.J. Aalbers, N.F.M. Kok, G.L. Beets, B.H.W. Hendriks, H.J.C.M. Sterenborg, K.F.D. Kuhlmann, T.J.M. Ruers, "Using diffuse reflectance spectroscopy to distinguish tumor tissue from fibrosis in rectal cancer patients as a guide to surgery", Accepted by Lasers in Surgery and Medicine, 2019

\section{PRESENTATIONS}

2015 NVvTG, Amsterdam

2017 SPIE Photonics West, San Francisco

2019 SPIE Photonics West, San Francisco

\section{POSTERS}

2017 EXCITE summer school, Zurich

2018 European Society of Surgical Oncology, Budapest

\section{COURSES}

2016 Coursera course on machine learning

2016 Good clinical practice

2016 BROK

2017 PerClass course on machine learning

2017 Technical writing and editing

2017 EXCITE summerschool

2018 Oncology course cancer center Amsterdam

$2018 \mathrm{iQ}$ winterschool on deeplearning 


\section{Curriculum Vitae}

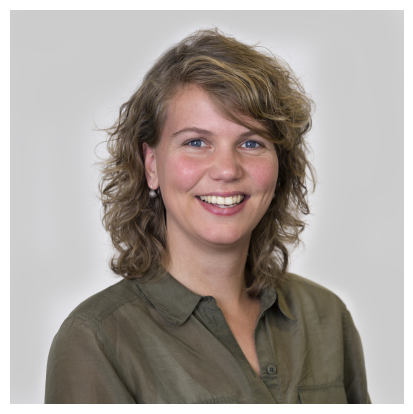

Lisanne (Elisabeth Johanna Maria) Baltussen was born in Rosmalen on June 4th 1989. She grew up in Monster were she finished primary school. After finishing secondary school in The Hague, she went to Enschede, Twente University, to study Technical Medicine. She finished her Bachelor in three years, after which she became board member of UniPartners Twente for one year. During her Master, Robotics and Imaging, she specialized in image processing. She completed five internships in four different hospitals in the final two years of her Master. Her final internship was a collaboration between Philips Research and the surgery department of the Netherlands Cancer Institute, in which she performed research on navigation during laparoscopic surgery. She started working as account manager in a start-up company but after a few months she returned to the Netherlands Cancer Institute to start her $\mathrm{PhD}$ candidacy on the use of diffuse reflectance spectroscopy in colorectal cancer surgery. 

